\title{
Ensemble Kalman filtering for hydraulic conductivity \\ characterization: parallelization and non-Gaussianity
}

\author{
$\mathrm{PhD}$ Thesis submitted by \\ Teng $\mathrm{Xu}$ \\ Advisor: \\ J. Jaime Gómez-Hernández
}

October 2014 



\title{
Ensemble Kalman filtering for hydraulic conductivity characterization: parallelization and non-Gaussianity
}

\author{
PhD Thesis submitted by \\ Teng $\mathrm{Xu}$ \\ Advisor: \\ J. Jaime Gómez-Hernández \\ Department: \\ Ingeniería Hidráulica y Medio Ambiente \\ Universitat Politècnica de València
}

October 2014, Valencia, Spain
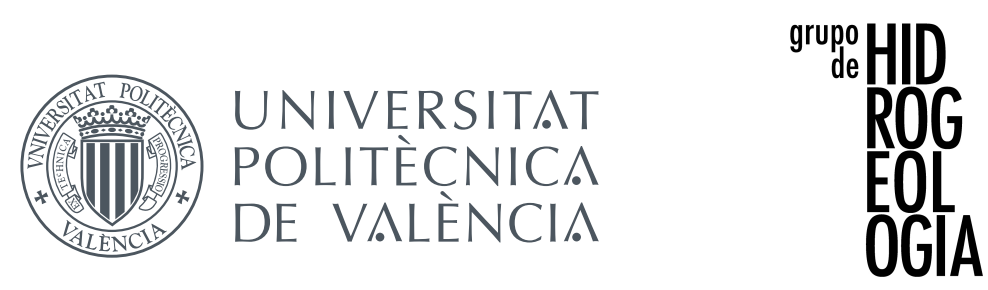
(c) Copyright by Teng Xu 2014

All rights reserved. 
To my family 


\section{Abstract}

Ensemble kalman filter (EnKF) has proven to be a powerful inverse method for the characterization of hydraulic conductivities, which works well for non-linear state equation and Gaussian-distributed parameters. It is computationally more efficient than other inverse methods; however, it is still time-consuming. This thesis addresses two issues, how to speed up the EnKF through code parallelization and how to properly address the problem of characterizing non-Gaussian hydraulic conductivity fields. It is organized in four parts.

The first part deals with the the parallelization of the ensemble Kalman filter (EnKF) algorithm in the context of hydraulic conductivity characterization using transient piezometric head data. The EnKF consists of two steps, forecast and analysis, and both steps have potential to be parallelized. In the forecast step, due to the Monte Carlo nature of the EnKF, the most straightforward way for parallelization is at the realization level, where each member of the ensemble is sent to a different processor. While in the analysis step, the computations of the covariances are distributed between the different processors. The results of speedup and efficiency show that the parallelized EnKF can significantly reduce the computation time, especially for a large number of ensemble realizations.

The second part describes an application of the localized normal-score EnKF with covariance inflation in a heterogenous bimodal hydraulic conductivity field. The objective is to demonstrate the power of transient piezometric head for the characterization of the spatial variability of a channelized bimodal hydraulic conductivity field, where the only existing prior information about conductivity is its univariate marginal distribution. Besides, covariance localization and covariance inflation are used to overcome the appearance of spurious correlations and the underestimation of the final uncertainty by the NS-EnKF. Covariance localization eliminates the effect of spurious correlations between state variables and parameters by constraining the correlation range of the empirical covariance. Covariance inflation is a technique used to avoid filter inbreeding by inflating the empirical covariance. The results show the importance of covariance localization and covariance inflation to reduce filter inbreeding.

The third part investigates the inverse method proposed by (Hu et al., 2013) and proposes an improved version. Unlike the idea of (Hu et al., 2013), 
which uses the EnKF to directly update uncorrelated uniform random fields (those used to draw from the local conditional marginal distributions in sequential simulation), the new version propose working on correlated uniform random fields, more precisely the same uniform random field used in probability field simulation (Froidevaux, 1993). The comparison of both versions shows that the new proposed one is much better than the original in order to capture the main patterns of conductivity and in reducing uncertainty.

The fourth and last part proposes a new stochastic inverse method named inverse sequential simulation (iSS). The iSS is a breed of sequential simulation and the normal-score ensemble Kalman filter. The new approach applies the ensemble concept to generate realizations by sequential simulation using the experimental non-stationary cross-covariance between conductivities and piezometric heads computed on an ensemble of realizations. We use the normal-score transformation to ensure marginal Gaussian distribution. And then, we apply standard multivariate sequential Gaussian conditional simulation to generate conductivity realizations conditioned to both conductivity and piezometric data. The benchmark against the NS-EnKF shows that the iSS is capable of generating inverse-conditioned non-Gaussian realizations with similar quality for both approaches. 


\section{Resumen}

El filtro de Kalman de conjuntos (EnKF) es un método inverso de gran potencial para la caracterización de conductividades hidráulicas, que funciona bien con ecuaciones de estado no lineales y parámetros con distribuciones gausianas. Es computacionalmente más eficiente que otros métodos inversos; sin embargo, consume mucho tiempo de cálculo. Esta tesis se centra en dos cuestiones, la forma de acelerar el EnKF a través de la paralelización del código y la forma de abordar adecuadamente el problema de la caracterización de campos de conductividad hidráulica no gausianos. La tesis está organizada en cuatro partes.

En la primera parte se presenta el algoritmo de paralelización del filtro de Kalman de conjuntos en el contexto de la caracterización de la conductividad hidráulica utilizando datos de piezometría en estado transitorio. El EnKF consta de dos pasos, predicción y análisis, y ambos pasos son susceptibles de ser paralelizados. En la fase de predicción, debido a que el EnKF tiene una componente importante de análisis de Monte-Carlo, la forma más sencilla para su paralelización es a nivel de realización, donde cada miembro del conjunto se envía a un procesador diferente. Mientras que en la etapa de análisis, los cálculos de las covarianzas se distribuyen entre los diferentes procesadores. Los resultados, en cuanto a aceleración y eficiencia del cálculo, muestran que la paralelización del EnKF puede reducir significativamente el tiempo de cálculo, especialmente para los casos en que el número de realizaciones es grande.

La segunda parte describe una aplicación del EnKF con anamorfosis localizada, e inflación de la covarianza a un campo de conductividades heterogéneo y con una distribución bimodal. El objetivo de esta parte es demostrar el potencial de las alturas piezométricas transitorias para la caracterización de la variabilidad espacial de un campo de conductividades hidráulicas bimodal y con patrones de variabilidad complejos, donde la única información a priori existente es su distribución marginal univariada. Además, se demuestra el uso de las técnicas de localización e inflación de la covarianza para evitar la aparición de correlaciones espurias y la subestimación de la incertidumbre final. La localización de la covarianza elimina el efecto de las correlaciones espurias entre las variables de estado y los parámetros al restringir el rango de correlación de la covarianza empírica. La inflación de la covarianza es una técnica utilizada para evitar el colapso 
del filtro aumentando (inflando) los valores de la covarianza empírica. Los resultados muestran la importancia de estas técnicas en la reducción del colapso del filtro.

La tercera parte investiga el método inverso propuesto por ( $\mathrm{Hu}$ et al., 2013) y propone una versión mejorada. A diferencia de la idea de (Hu et al., 2013), que utiliza el EnKF para actualizar directamente campos uniformes no correlacionados (los que se utilizan para el muestreo aleatorio de las distribuciones marginales condicionales locales en la simulación secuencial), la nueva versión propone trabajar en campos aleatorios uniformes correlacionados, más precisamente los mismos campos que se utilizan en la simulación por campos de probabilidades (Froidevaux, 1993). La comparación de las dos versiones demuestra que la nueva versión propuesta es mucho mejor que la original tanto a la hora de capturar los principales patrones de conductividad como en la reducción de la incertidumbre.

La cuarta y última parte propone un nuevo método inverso estocástico llamado simulación secuencial inversa (iSS). La iSS es una mezcla de simulación secuencial y filtro de Kalman con anamorfosis. El nuevo método usa el concepto de conjuntos del EnKF para generar realizaciones por simulación secuencial usando las covarianzas cruzadas no estacionarias entre conductividades experimentales y alturas piezométricas calculadas a partir de un conjunto de realizaciones. Utilizamos la anamorfosis para asegurar una distribución gausiana marginal. Y entonces, aplicamos la simulación secuencial gausiana estándar para generar realizaciones de conductividad condicionando tanto en la conductividad como en los datos piezométricos. El comparación con el NS-EnKF muestra que la iSS es capaz de generar realizaciones no gausianas condicionadas a las alturas piezométricas con calidad similar para ambos métodos. 


\section{Resum}

El filtre de Kalman de conjunts (EnKF) és un mètode invers de gran potencial per a la caracterització de conductivitats hidràuliques, que funciona bé amb equacions d'estat no lineals i paràmetres amb distribucions gausianes. És computacionalment més eficient que altres mètodes inversos; no obstant açò, consumeix molt temps de càlcul. Aquesta tesi se centra en dues qüestions, la forma d'accelerar el EnKF a través de la paralelització del codi i la forma d'abordar adequadament el problema de la caracterització de camps de conductivitat hidràulica no gausians. La tesi està organitzada en quatre parts.

En la primera part es presenta l'algorisme de paralelitzación del filtre de Kalman de conjunts en el context de la caracterització de la conductivitat hidràulica utilitzant dades de piezometria en estat transitori. El EnKF consta de dos passos, predicció i anàlisi, i tots dos passos són susceptibles de ser paralelitzats. En la fase de predicció, a causa que el EnKF té una component important d'anàlisi de Monte-Carlo, la forma més senzilla per a la seua paralelització és a nivell de realització, on cada membre del conjunt s'envia a un processador diferent. Mentre que en l'etapa d'anàlisi, els càlculs de les covariàncies es distribueixen entre els diferents processadors. Els resultats, quant a acceleració i eficiència del càlcul, mostren que la paraleliztació del EnKF pot reduir significativament el temps de càlcul, especialment per als casos en què el nombre de realitzacions és gran.

La segona part descriu una aplicació del EnKF amb anamorfosis localitzada, i inflació de la covariància a un camp de conductivitats heterogeni i amb una distribució bimodal. L'objectiu d'aquesta part és demostrar el potencial de les altures piezométriques transitòries per a la caracterització de la variabilitat espacial d'un camp de conductivitats hidràuliques bimodal i amb patrons de variabilitat complexos, on l'única informació a priori existent és la seua distribució marginal univariada. A més, es demostra l'ús de les tècniques de localització i inflació de la covariància per a evitar l'aparició de correlacions espúries i la subestimació de la incertesa final. La localització de la covariància elimina l'efecte de les correlacions espúries entre les variables d'estat i els paràmetres en restringir el rang de correlació de la covariància empírica. La inflació de la covariància és una tècnica utilitzada per a evitar el col-lapse del filtre augmentant els valors de la covariància 
empírica. Els resultats mostren la importància d'aquestes tècniques en la reducció del col-lapse del filtre.

La tercera part investiga el mètode invers proposat per (Hu et al., 2013) i proposa una versió millorada. A diferència de la idea de (Hu et al., 2013), que utilitza el EnKF per a actualitzar directament camps uniformes no correlacionats (els que s'utilitzen per al mostreig aleatori de les distribucions marginals condicionals locals en la simulació seqüencial), la nova versió proposa treballar en camps aleatoris uniformes correlacionats, més precisament els mateixos camps que s'utilitzen en la simulació per camps de probabilitats (Froidevaux, 1993). La comparació de les dues versions demostra que la nova versió proposada és molt millor que l'original tant a l'hora de capturar els principals patrons de conductivitat com en la reducció de la incertesa.

La quarta i última part proposa un nou mètode invers estocàstic anomenat simulació seqüencial inversa (iSS). La iSS és una mescla de simulació seqüencial i filtre de Kalman amb anamorfosis. El nou mètode usa el concepte de conjunts del EnKF per a generar realitzacions per simulació seqüencial usant les covariàncies creuades no estacionàries entre conductivitats experimentals i altures piezométriques calculades a partir d'un conjunt de realitzacions. Utilitzem la anamorfosis per a assegurar una distribució gaussiana marginal. I llavors, apliquem la simulació seqüencial gaussiana estàndard per a generar realitzacions de conductivitat condicionant tant en la conductivitat com en les dades piezométriques. El comparació amb el NS-EnKF mostra que la iSS és capaç de generar realitzacions no gausianes condicionades a les altures piezométriques amb qualitat similar per a tots dos mètodes. 


\section{Acknowledgements}

I would like to send my deepest gratitudes to my advisor Prof. J. Jaime Gómez-Hernández for giving me this chance to pursue my $\mathrm{PhD}$ studies at Universitat Politècnica de València. He is so knowledgeable and professional that, when I am stuck in the research work, he can always help me to find a way out and give me valuable suggestions. I deeply appreciates his instructions and kindness.

The financial support of my PhD studies comes mainly from the China Scholarship Council (CSC No. 2010640054). Financial support to carry out the studies was also received from the Spanish Ministry of Science and Innovation through project CGL2011-23295. I have also received funding from Universitat Politècnica de València for a two-month stay at the Kansas Geological Survey. All of these financial supports are gratefully acknowledged.

Here, typically, I would like to express my great thanks to Liangping and Haiyan for their kind help both in my life and study in my first one and a half years in Valencia. I will never forget the special day when I arrived at the Valencia airport, they picked me up and helped me find a nice house. Thanks to them, I could adapt myself to a new life in Valencia sooner and faster. Besides, my sincere thanks goes to Butler and Gaisheng for their kind help during the period I visited to the Kansas Geologic Survey.

I also want to thank Julio and Amanda for helping me dealing with everyday matters in the university. I really appreciate the invitations from Julio to have Christmas dinner with his family and friends in these years. Besides, I also want to say thanks to the colleagues in the research group and in the department, Eduardo, Andrés, Javier, Óscar, Teresa, and Julio Núñez. It will remain as a precious memory for me to have worked together with you. Many thanks are also given to my friends I made in these four years. Without you, my life would not be so fruitful and colorful.

At the end, I would like to send my sincere thanks to my family. Their support really means a lot to me. 


\title{
Contents
}

\begin{abstract}
$\quad$ v
Resumen vii

Resum ix

Acknowledgements $\quad$ xi

1 Introduction $\quad 1$

1.1 Motivation and Objectives . . . . . . . . . . . . . 1

1.2 Thesis Organization . . . . . . . . . . . . 2
\end{abstract}

2 Parallelized Ensemble Kalman Filter for Hydraulic Conductivity Characterization (published in Computers \& Geosciences) $\mathbf{5}$

2.1 Motivation . . . . . . . . . . . . . 6

2.2 Overview of the EnKF . . . . . . . . . . . 7

2.3 Parallelization approach . . . . . . . . . . . . 10

2.3 .1 Forecast step . . . . . . . . . . . . . . . 10

2.3 .2 Update step . . . . . . . . . . . . . . . . . . 12

2.4 Application . . . . . . . . . . . . . . . . . . . . . . 12

2.4 .1 Analysis . . . . . . . . . . . . . . . 14

2.4 .2 Parallelization analysis . . . . . . . . . . . . . . . 15

2.5 Summary and suggestion . . . . . . . . . . . . 20

3 The Power of Transient Piezometric Head Data in Inverse Modeling: An Application of the Localized Normal-score EnKF with Covariance Inflation in a Heterogenous Bimodal Hydraulic Conductivity Field (published in Advances in Water Re$\begin{array}{ll}\text { sources) } & 21\end{array}$

3.1 Introduction . . . . . . . . . . . . . . . . 22

3.2 The Localized Normal-Score Ensemble Kalman Filter with Covariance Inflation . . . . . . . . . . . . . . 24

3.3 Synthetic Example . . . . . . . . . . . . . . . . . 27

3.3 .1 Scenarios . . . . . . . . . . . . . 28

3.4 Analysis . . . . . . . . . . . . . . . . . . . . 32 
3.5 Summary and conclusion . . . . . . . . . . . . 52

4 Probability Fields Revisited in the Context of Ensemble Kalman Filtering (to be submitted)

4.1 Introduction . . . . . . . . . . . . . . 56

4.2 Methodology . . . . . . . . . . . . . 58

4.2.1 Uncorrelated probability field method . . . . . . . . 58

4.2.2 Correlated probability field method . . . . . . . . . 59

4.3 Synthetic Example . . . . . . . . . . . . . . . . . 60

4.4 Reproducing log-conductivity . . . . . . . . . . . . . 63

4.5 Reproducing piezometric head . . . . . . . . . . . . 72

4.6 Discussion . . . . . . . . . . . . . . . . . . 74

5 Inverse sequential simulation: A new approach for the characterization of non-Gaussian hydraulic conductivity fields (submitted to Water Resources Research) $\quad \mathbf{7 5}$

5.1 Introduction . . . . . . . . . . . . . 76

5.2 Methodology . . . . . . . . . . . . . . . . 77

5.3 Synthetic Example . . . . . . . . . . . . . . . . 82

5.4 Analysis . . . . . . . . . . . . . . . . . . 85

5.5 Discussion . . . . . . . . . . . . . . . . . . . 94

5.6 Summary and conclusions . . . . . . . . . . . . . 94

6 Inverse Sequential Simulation: Performance and Implementation Details (submitted to Advances in Water Resources) 97

6.1 Introduction . . . . . . . . . . . . . . . . . . 98

6.2 Methodology . . . . . . . . . . . . . . . . . . 99

6.3 Synthetic Example . . . . . . . . . . . . . . . . . . . 102

6.4 Analysis . . . . . . . . . . . . . . . . . . . 107

6.4.1 Conditioning to heads . . . . . . . . . . . . . 107

6.4.2 Reproduction of conductivities . . . . . . . . 108

6.4.3 Postaudit . . . . . . . . . . . . . . . . . 114

6.4 .4 Discussion . . . . . . . . . . . . . 116

$\begin{array}{llr}7 \text { Conclusions } & 121\end{array}$

7.1 Summary . . . . . . . . . . . . . . . . . . . 121

7.2 Suggestions for Future Research . . . . . . . . . . . . . . 122

$\begin{array}{ll}\text { Bibliography } & 125\end{array}$

$\begin{array}{ll}\text { Appendix } & 137\end{array}$

$\begin{array}{ll}\text { A Normal-score transform } & 137\end{array}$ 


\section{List of Figures}

2.1 The parallel EnKF flow chart: box 1 is for the forecast step; box 2 is for the updating step. . . . . . . . . . . . 11

2.2 Flow domain and location of the 75 observation wells in the small model. . . . . . . . . . . . . . . . . . . 13

2.3 Flow domain and location of the 25 observation wells in the large model. The filled triangles are observation wells measuring the piezometric heads at the first, third and fifth layers, and the filled circle is a verification well. . . . . . . . . 14

2.4 Reference realization of $\ln (k) \ldots \ldots \ldots \ldots \ldots$

2.5 initial and updated ensemble means facies fields.(a), (b) show the ensemble mean facies fields of the initial realizations and the 50th updated realizations of test $1 ;$ (c), (d) show the ensemble mean facies fields of the initial realizations and the 50 th updated realizations for test 2 ; (e), (f) show the ensemble mean facies fields of the initial realizations and the 50th updated realizations for test $3 . \ldots \ldots \ldots 16$

2.6 Piezometric head time evolution at the control well. The red curve is for the reference field, the gray curves for each realization of the ensemble, and the green curve is the ensemble mean. (a) and (b) show piezometric heads of the the initial realizations and the 50th updated realizations for test 1 ; (c) and (d) show piezometric heads of the initial realizations and the 50 th updated realizations for test 2 ; (e) and (f) show piezometric heads of the initial realizations and the 50th updated realizations for test $3 . \ldots \ldots \ldots$. . . . . . . . . 17

2.7 The root mean square error for three test. . . . . . . . . . 18

2.8 Parallel speed-up and efficiency. (a) Speed up (b) efficiency. . 19

3.1 Training image used to generate the ensemble of binary facies realizations. . . . . . . . . . . . . 28

3.2 Reference field. The line source where particles are injected is shown with the blue dashed line, the two control planes indicated by the black lines are used to compute breakthrough curves. . . . . . . . . . . . . . . . 29 
3.3 The histogram of the reference field . . . . . . . . . . 29

3.4 The localization function . . . . . . . . . . . . 31

3.5 Graphs a,b,c show the locations of the 111, 56, 24 observation piezometers, respectively. The blue squares denote observation piezometers, and the red triangles, control piezometers. .

3.6 Scenario S0. Log-conductivity histograms for the initial ensemble of realizations and for the updated ensemble of realizations after the 60 th assimilation step. . . . . . . . . .

3.7 Scenarios S1-S6. Log-conductivity histograms. The top one shows the histogram for the initial ensemble of homogeneous realizations used in scenarios S1-S6; graphs a to f show the histograms for the corresponding scenarios after the 60th data assimilation step. The scenarios without covariance inflation are shown in the left column; the scenarios with covariance inflation are shown in the right column. The number of observation piezometers used is indicated in the left. . . . . . .

3.8 Scenario S0. Ensemble mean of $\ln K$ for the initial realizations and after assimilating heads at the 10th and 60th time steps.

3.9 Scenario S0. Ensemble $\ln K$ variance for the initial realizations and after assimilating heads at the 10th and 60th time steps. . . . . . . . . . . . . . .

3.10 Maps a,b show the ensemble mean and ensemble variance of the initial realizations for the scenarios with initial homogenous fields $(\mathrm{S} 1-\mathrm{S} 6) \ldots \ldots \ldots \ldots \ldots$

3.11 Scenarios S1-S6. Log-conductivity ensemble mean computed after the 10th time step. . . . . . . . . . . . . 36

3.12 Scenarios S1-S6. Log-conductivity ensemble mean computed after the 60th time step. . . . . . . . . . . . 37

3.13 Scenarios S1-S6. Log-conductivity ensemble variance computed after the 10 th time step. . . . . . . . . . 38

3.14 Scenarios S1-S6. Log-conductivity ensemble variance computed after the 60 th time step. . . . . . . . . . . 39

$3.15 R M S E \ldots \ldots \ldots \ldots \ldots \ldots \ldots \ldots \ldots$

3.16 The ratio of $R M S E$ to $S E \ldots \ldots \ldots \ldots \ldots \ldots$

3.17 Graphs a,b show the piezometric head time evolution of the initial ensemble of heterogenous log-conductivity realizations at the control piezometers 1 and 2, respectively; graphs c,d show the piezometric head time evolution on the initial homogenous realizations at the two control piezometers. The red square line corresponds to the piezometric head time evolution in the reference, the green triangle line corresponds to the mean of the ensemble, and the gray lines correspond to the realizations. . . . . . . . . . . . . . 
3.18 Scenario S0. The piezometric head time evolution after the 60 th time step for the two control piezometers. The red square line corresponds to the piezometric head time evolution in the reference, the green delta line corresponds to the mean of the ensemble, the gray lines correspond to the realizations and the vertical dashed lines indicate the conditioning period. . . . . . . . . . . . . . . . 44

3.19 Scenarios S1-S6. The piezometric head time evolution at the control piezometer 1 after the 60th time step. The red square line corresponds to the piezometric head time evolution in the reference, the green delta line corresponds to the mean of the ensemble, the gray lines correspond to the realizations and the vertical dashed lines indicate the conditioning period. .

3.20 Scenarios S1-S6. The piezometric head time evolution at the control piezometer 2 after the 60th time step. The red square line corresponds to the piezometric head time evolution in the reference, the green delta line corresponds to the mean of the ensemble, the gray lines correspond to the realizations and the vertical dashed lines indicate the conditioning period. . . 46

3.21 Graphs a,b show the BTCs at the two control planes for scenario S0. The red square line corresponds to BTCs in the reference. The black lines correspond to the 5 and 95 percentiles of all realization BTCs, and the green delta line corresponds to the median. . . . . . . . . . . . . . .

3.22 Scenarios S1-S6. The BTCs at the first control plane. The red square line corresponds to BTCs in the reference. The black lines correspond to the 5 and 95 percentiles of all realization BTCs, and the green delta line corresponds to the median.

3.23 Scenarios S1-S6. The BTCs at the second control plane. The red square line corresponds to BTCs in the reference. The black lines correspond to the 5 and 95 percentiles of all realization BTCs, and the green delta line corresponds to the median. . . . . . . . . . . . . . . . .

3.24 Scenarios S0. Connectivity curves for the initial ensemble of realizations and for the updated realizations after the 60th assimilation time step. The red square line corresponds to the connectivity curve in the reference, the green delta line corresponds to the mean of the ensemble, and the gray lines correspond to the individual realizations. . . . . . . . . . 50

3.25 Scenarios S1-S6. Connectivity curves. The red square line corresponds to connectivity curves in the reference, the green delta line corresponds to the mean of the ensemble, and the gray lines correspond to the individual realizations . . . . . . 
4.1 Work flow for the Unconditional Probability Field method. The starting step is highlighted in bold. . . . . . . . . . 58

4.2 Work flow for the Conditional Probability Field method. The starting step is highlighted in bold. . . . . . . . . . . . . 60

4.3 Training image . . . . . . . . . . . . . . . . 61

4.4 Synthetic aquifer and log-conductivity histogram. . . . . . 62

4.5 Distribution of observation piezometers . . . . . . . . . 62

4.6 Ensemble mean, ensemble variance and ensemble histogram of the initial ensemble of log-conductivity realizations. . . . . 64

4.7 Ensemble mean, ensemble variance and ensemble histogram of the log-conductivity realizations obtained after 10 assimilation time steps. Left column, uncorrelated probability fields (S1). Right column, correlated ones (S2).

4.8 Ensemble mean, ensemble variance and ensemble histogram of the log-conductivity realizations obtained after 60 assimilation time steps. Left column, uncorrelated probability fields (S1). Right column, correlated ones (S2).

4.9 Evolution of the 400th realization in both methods. Top row, uncorrelated probabilities (S1); bottom row, correlated probabilities (S2). Columnwise from left to right, initial realization (same for both approaches), after time step 9, 10, 59 and $60 \ldots \ldots \ldots \ldots \ldots \ldots \ldots \ldots \ldots \ldots \ldots \ldots \ldots \ldots \ldots \ldots$

4.10 Evolution of the 800th realization in both methods. Top row, uncorrelated probabilities (S1); bottom row, correlated probabilities (S2). Columnwise from left to right, initial realization (same for both approaches), after time step 9, 10, 59 and $60 \ldots \ldots \ldots \ldots \ldots \ldots \ldots$

4.11 Increment of Gaussian deviates in realization 400. Top row, uncorrelated probabilities (S1); bottom row, correlated probabilities (S2). Left column, update at the 10th step; right column, update at the 60 th step. . . . . . . . . . . . .

4.12 Increment of Gaussian deviates in realization 800. Top row, uncorrelated probabilities (S1); bottom row, correlated probabilities (S2). Left column, update at the 10th step; right column, update at the 60 th step. . . . . . . . . . . . . .

4.13 Faces changes induced by updating the probabilities in the uncorrelated probability field method. Blue means change from sand to shale, red means change from shale to sand, and green means no change. Right column for the updates at time step 10, left column for the updates at time step 60, top row for realization 400 , bottom row for realization $800 \ldots$. . . 
4.14 Reproduction of the observed piezometric head at the piezometers \#1 and \#2 of Figure 4.5 by the log-conductivity fields obtained after 60 time steps. Left, piezometer \#1; right, piezometer \#1. The red squares correspond to the head evolution in the synthetic aquifer, the gray lines are the head evolution in the individual realizations, and the green triangles correspond to the ensemble mean. . . . . . . . . . . .

4.15 Reproduction of the observed piezometric head at the piezometers \#1 and \#2 of Figure 4.5 by the log-conductivity fields obtained after 60 time steps for both methods. Top row, uncorrelated probability field method (S1); bottom row, correlated probability field method (S2). Left, piezometer \#1; right, piezometer \#1. Meaning of lines same as previous figure. 73

5.1 Location of the conditional data. The red nodes denote shale; the green nodes denote sand. . . . . . . . . . . . 83

5.2 Training image used to generate the ensemble of binary facies realizations. . . . . . . . . . . . . 83

5.3 Reference field. It shows the boundary conditions; it also shows the source line (dashed) and the control planes at which breakthrough curves are computed (solid lines) in the transport experiment. . . . . . . . . . . . . . 84

5.4 The histogram of the reference log-conductivity field . . . . 84

5.5 Location of wells. Red triangles denote observation wells; green squares denote injection (\#1, \#2) and pumping wells (\#3, \#4 and \#5). The observation wells \#6, \#7 are used as

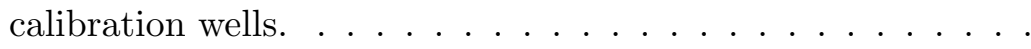

5.6 Log-conductivity histograms of the initial ensemble of realizations and of the updated ensemble of realizations after the 50th assimilation step of the two scenarios. . . . . . . 86

5.7 Ensemble mean and ensemble variance of $\ln K$ for the initial realizations. . . . . . . . . . . . . 87

5.8 Ensemble mean of $\ln K$ after assimilating observation heads at the 10th and 50th time steps for the two scenarios. . . . 88

5.9 Ensemble variance of $\ln K$ after assimilating observation heads at the 10th and 50th time steps for the two scenarios. . . . . 89

5.10 RMSE, ES, and RMSE/ES. . . . . . . . . . . . . 90

5.11 Evolution in time of the piezometric head at the two calibration wells for the initial ensemble of log-conductivity realizations. The red square line corresponds to the piezometric head in the reference, the gray lines correspond to the realizations and the vertical dashed lines marks the end of the conditioning period, the green delta line corresponds to the average of the gray lines. . . . . . . . . . . . . 
5.12 Evolution in time of the piezometric head at the two calibration wells after the 50th time step. The red square line corresponds to the piezometric head in the reference, the gray lines correspond to the realizations and the vertical dashed lines marks the end of the conditioning period, the green delta line corresponds to the average of the gray lines. . . . . . . . . .

5.13 Breakthrough curves (BTC) at the two control planes for the initial $\ln K$ realizations. The red square line corresponds to the BTCs in the reference. The black lines correspond to the 5 and 95 percentiles of all realization BTCs, and the green delta line corresponds to the median. . . . . . . . . . . .

5.14 Breakthrough curves (BTC) at the two control planes for the $\ln K$ realizations updated after the 50 th time step. The red square line corresponds to BTCs in the reference. The black lines correspond to the 5 and 95 percentiles of all realization BTCs, and the green delta line corresponds to the median.

6.1 Training image used to generate the ensemble of binary facies realizations . . . . . . . . . . . . . . 103

6.2 Location of the facies data, red nodes for shale and green nodes for sand . . . . . . . . . . . . . . . 104

6.3 Reference field . . . . . . . . . . . . . . . . . . . . . 104

6.4 Histogram of the reference field . . . . . . . . . . . . 104

6.5 Location of the facies data for scenario S3, the one with a larger number of conditioning points; red nodes for shale and green nodes for sand . . . . . . . . . . . . . 106

6.6 (a) Distribution of piezometers of scenarios S1, S2, S3, S4 and S6; (b) Distribution of piezometers for scenario S5. For reference, the injection $(\# 1, \# 2)$ and pumping wells (\#3, \#4 and \#5) are also shown. The piezometric head evolution at piezometers \#6, \#7 is used in the analysis. . . . . . . . 106

6.7 Evolution of piezometric head at piezometers \#6 (top row) and \#7 (bottom row) on the initial sets of realizations for the different scenarios. Scenarios S1, S4 and S5 share the same set of initial realizations (first column), scenarios S2, S3 and S6 are shown in the second, third and fourth columns, respectively. The gray lines correspond to the evolution in each realization. The red line is the piezometric evolution in the reference, and the green line is the time average of all gray lines. The vertical dashed lines indicate the end of the conditioning data. . . . . . . . . . . . . 108 
6.8 Evolution of piezometric head at piezometers \#6 (top two rows) and \#7 (bottom two rows) on the sets of realizations generated by iSS after conditioning to piezometric heads during the first 50 time steps (vertical dashed line) for the different scenarios. Meaning of lines is the same as previous figure. . . . . . . . . . . . . . . . 109

6.9 Evolution of piezometric head at piezometers \#6 (top row) and \#7 (bottom row) on the sets of realizations generated by iSS for scenario S1 at three different times: initial, after 10 time steps, and after 50 time steps. Meaning of lines is the same as previous figure. . . . . . . . . . . . . . 110

6.10 Ensemble mean (top row) and ensemble variance (bottom row) of logconductivity for the sets of initial realizations for the different scenarios. First column is for scenarios S1, S4 and S5, second column for S2, third column for S3, and fourth column for S6. . . . . . . . . . . . . . . . . . . 11

6.11 Ensemble mean (top two rows) and ensemble variance (bottom two rows) of logconductivity for the sets of realizations for the different scenarios obtained after 50 time steps. . . . . 112

6.12 Ensemble mean (top row) and ensemble variance (bottom row) of logconductivity for the sets of realizations generated by iSS for scenario S1 at three different times: initial, after 10 time steps, and after 50 time steps. . . . . . . . . . 112

6.13 Histograms of logconductivity for the sets of realizations for the different scenarios obtained after 50 time steps. . . . . . 113

6.14 Ensemble mean (top row) and ensemble variance (bottom row) of the ensemble of normal scores of logconductivity as generated by the sequential simulation algorithm for scenario S1 after 50 time steps, and of the corresponding log-conductivity fields after backtransforming the normal-scores. . . . . . . . . 114

6.15 RMSE and ES of all the scenarios. . . . . . . . . . . . 115

6.16 Solute breakthrough curves (BTC) at control planes 1 (top row) and 2 (bottom row) on the initial sets of realizations used in the different scenarios. The red line is the BTC in the reference, the green line is the median (computed timewise) of the BTCs from the different realizations, and the two black lines mark the $90 \%$ confidence interval bounded by the $5 \%$ and $95 \%$ percentiles. . . . . . . . . . . . 116

6.17 Solute breakthrough curves (BTC) at control planes 1 (top two rows) and 2 (bottom two rows) on the sets of realizations obtained for the different scenarios generated after 50 time steps. Meaning of lines is the same as in previous figure. . . . 117 
6.18 Solute breakthrough curves (BTC) at control planes 1 (top row) and 2 (bottom row) for scenario S1 on the initial set of realizations, those generated after 10 time steps, and those generated after 50 time steps. Meaning of lines is the same as in previous figure. . . . . . . . . . . . . 118 


\section{List of Tables}

2.1 Parallel performance of three of the tests. . . . . . . . . . 19

3.1 Parameters of the random functions describing the spatial continuity of the sand and shale logconductivities . . . . 28

3.2 Definition of scenarios . . . . . . . . . . . . 30

4.1 Parameters of the random functions describing the spatial continuity of the sand and shale log-conductivities . . . . . 61

5.1 Parameters of the random functions describing the spatial continuity of the sand and shale log-conductivities . . . . . 82

6.1 Parameters of the random functions describing the spatial continuity of the sand and shale log-conductivities . . . . . 103

6.2 Definition of scenarios . . . . . . . . . . . . 105 



\section{1 \\ Introduction}

\subsection{Motivation and Objectives}

As we know, reliable groundwater flow and transport modeling are very important for groundwater forecast, solute migration forecast, subsurface resource management, and environmental risk assessment. The quality of groundwater flow and transport modeling is mostly dependent on a proper characterization of the subsurface hydrogeological properties (such as hydraulic conductivity, porosity, etc.) However, in reality, the experimental measurements of these properties are very sparse compared to the scale of the aquifers. So, how to use these sparse data to get the best characterization possible of the subsurface hydrogeologic properties is a real challenge.

In recent decades, stochastic inverse modeling accounting for real-time state data has proven to be a powerful technique for the characterization of subsurface hydrogeologic properties. Some existing methods for stochastic inverse modeling are the gradual deformation method $(\mathrm{Hu}, 2000)$, the sequential self-calibration (Gómez-Hernánez et al., 1997), the Markov chain Monte Carlo method Oliver et al. (1997), the representer method (Valstar et al., 2004), the pilot points method (De Marsily et al., 1984), and the ensemble Kalman filter (EnKF) (Evensen, 2003). Among these methods, the EnKF is getting more popular as a very effective inverse method in recent years. However, large computational needs and the difficulty to characterize non-Gaussian parameter fields are major drawbacks to be addressed.

There are many researchers working on the solution of the above-mentioned issues. To address the problem of computational efficiency, the EnKF ha been coupled with covariance localization and with upscaling to reduce the 
ensemble size and to speed up the solution of the state equation ( $\mathrm{Li}$ et al., 2012c). An analysis of the EnKF algorithm itself shows that it can be parallelized resulting in savings of running time. To address the problem of how to handle non-Gaussian fields, several approaches have been developed; for example, the methods using a Gaussian mixture model (Dovera and Della Rossa, 2011), the methods using normal score transform (Zhou et al., 2011), the methods using transformed reparameterization (Chen et al., 2009), and the iterative EnKF ( $\mathrm{Gu}$ and Oliver, 2007). The normal-score EnKF proposed by Zhou et al. (2011) has proven to be one of the most effective methods to deal with non-Gaussian distributions. Moreover, Zhou et al. (2011) showed that transient piezometric head was enough for hydraulic conductivity characterization if a training image for the hydraulic conductivity was available. We will go even further and analyze what if there is no information about conductivity except for its univariate marginal distribution, can we still get a proper characterization from transient piezometric head data only?

Taking as inspiration the normal-score EnKF algorithm, and trying to go beyond the incremental updating of realizations implicit in the ensemble Kalman filter, we have developed a new inverse algorithm by blending some of the ideas underlying multivariate Gaussian sequential simulation and ensemble Kalman filtering; this new method is capable of dealing with non-Gaussian distribution.

The main objectives of this thesis can be summarized as follows: First, design a new parallelized algorithm for the EnKF. Second, analyze the power of transient piezometric head data in the characterization of non-Gaussian parameter fields using the localized normal-score ensemble Kalman filter coupled with covariance inflation with the only information on conductivities of its univariate marginal distribution. And third, develop a new stochastic inverse modeling approach based on sequential simulation to characterize non-Gaussian parameter fields.

\subsection{Thesis Organization}

Aside from the motivation and objectives, the rest of the thesis is organized as follows: The fist five chapters are independent papers which are published or under review or to be submitted in refereed international journals. And the last chapter is the summary of the whole work.

Chapter 2 presents a new parallelized EnKF algorithm. Four tests with different ensemble size and model size are designed to evaluate the speedup and efficiency of the parallelized EnKF.

Chapter 3 proposes a localized NS-EnKF with covariance inflation to reduce filter inbreeding and eliminate the effect of spurious correlations among the state variables and the parameters. A synthetic bimodal confined aquifer 
is designed and seven scenarios are used to demonstrate the effectiveness of the proposed approach. Besides, we also show the power of transient piezometric head information for the characterization of the spatial variability of hydraulic conductivity with the only information of univariate marginal distribution of hydraulic conductivities.

Chapter 4 presents an improved inverse method on the base of the method proposed by (Hu et al., 2013) to characterize non-Gaussian hydraulic conductivity fields. A synthetic channelized bimodal continuous hydraulic conductivity field is used to show and compare the improved method and previous method.

Chapter 5 proposes a new inverse modeling approach based on sequential simulation (iSS) to character non-Gaussian hydraulic conductivity fields. A synthetic bimodal confined aquifer is used to demonstrate the ability of the new method to characterize non-Gaussian fields. The performance of the proposed approach is compared with that of the NS-EnKF.

Chapter 6 applies the iSS to a synthetic channelized bimodal hydraulic conductivity field and six scenarios with difference number of conditional data, searching nodes and ensemble size with the aim of evaluating the performance and sensitivity of the iSS.

Chapter 7 summarizes all the works in this thesis and shows some suggestions for future research. 



\title{
2 \\ 2 \\ Parallelized Ensemble Kalman Filter for Hydraulic Conductivity Characterization
}

\begin{abstract}
The ensemble Kalman filter (EnKF) is nowadays recognized as an excellent inverse method for hydraulic conductivity characterization using transient piezometric head data. Its implementation is well suited for a parallel computing environment. A parallel code has been designed that uses parallelization both in the forecast step and in the analysis step. In the forecast step, each member of the ensemble is sent to a different processor, while in the analysis step, the computations of the covariances are distributed between the different processors. An important aspect of the parallelization is to limit as much as possible the communication between the processors in order to maximize execution time reduction.

Four tests are carried out to evaluate the performance of the parallelization with different ensemble and model sizes. The results show the savings provided by the parallel EnKF, especially for a large number of ensemble realizations.
\end{abstract}




\subsection{Motivation}

The ensemble Kalman filter (EnKF) proposed by Evensen (Evensen, 2003) has proven to be an effective inverse method. It has been applied in many fields such as petroleum engineering, oceanography, meteorology or hydrology (e.g., Aanonsen et al., 2009; Evensen, 2003; Dowell et al., 2004; Franssen and Kinzelbach, 2008; Li et al., 2012a). While it has proven to be more effective than alternative inverse methods (Hendricks Franssen and Kinzelbach, 2009), it still has important computational needs.

The ensemble Kalman filter is based on the simultaneous analysis of a large number of realizations. The first attempts to reduce CPU time usage were aimed at reducing the number of realizations in the ensemble. The covariance localization is a modification of the initial EnKF implementation that serves to reduce the ensemble size without compromising much the quality of the outcome (e.g., Houtekamer and Mitchell, 1998; Anderson, 2007b; Devegowda et al., 2010; Zhang and Oliver, 2010). Other authors have proposed to reduce the size of each realization, for instance, Li et al. (2012c) couple upscaling and the EnKF for the inverse modeling of groundwater flow.

In any case, even for the most efficient implementation of the EnKF, the fact that it works on each one of an ensemble of realizations makes it amenable to parallelization and thus take advantage of multi-processor computers or of grid computing to reduce even further the time needed for the algorithm to run. Although it will be reviewed in more detail later in the paper, recall that the EnKF takes an ensemble of realizations, runs a forward model in each realization, collects state data at observation locations and uses the difference between the predicted and observed values to update each one of the realizations. The updating step requires using all the ensemble realizations to compute the Kalman gain.

Keppenne (2000) proposed a parallel algorithm in which the forward model for each ensemble member is run in a different processor, then, to compute the Kalman gain, a domain decomposition is performed and each processor ends up with a small portion of all ensemble realizations, finally, for the updating, each processor is responsible of the update of a realization. (Keppenne and Rienecker, 2002a,b, 2003) also apply a domain decomposition in the updating step and each processor is responsible of updating the subdomain used for the Kalman gain computation in all realizations. This approach has the advantage of avoiding the ensemble transpositions across processor that would be required if, after the computation of the Kalman gain, each processor updates a full realization.

Recently, Tavakoli et al. (2011) have proposed a parallel EnKF algorithm applied to petroleum engineering using a three-level parallelization. On the first level, each ensemble member runs on a separate processor during the forecast step, on the second level uses a parallel implementation of the 
reservoir simulator, and, on the third level, it handles the matrix-vector multiplications involved in the Kalman gain computation and the updating of the ensemble members.

In this paper we propose an alternative parallel EnKF algorithm and provide the computer code to run it on a parallel environment using MODFLOW (McDonald and Harbaugh, 1988; Harbaugh et al., 2000) as the forward model. To the best of our knowledge this is the first application of a parallel EnKF algorithm in the field of hydrogeology.

The paper proceeds with an overview of the EnKF, the strategy for parallelization, and the evaluation of the algorithm on two examples.

\subsection{Overview of the EnKF}

The EnKF is the evolution of the Kalman filter to better address nonlinear state transfer functions using a Monte-Carlo approach. The Kalman filter was proposed by Kalman et al. (1960) as a data assimilation filter to improve the estimation of the state of a dynamic linear system. Later, the extended Kalman filter (EKF) was proposed to address nonlinear systems; the extension is based on a linearization of the nonlinear model, using a Taylor expansion, for the computation of the time evolution of the covariance (McElhoe, 1966). The EKF has been used in many fields, including hydrology (e.g., Hantush, 1997; Leng and Yeh, 2003; Yeh and Huang, 2005), however, it has severe shortcomings in dealing with highly nonlinear functions due to the accumulative error induced by the linearization process. Besides, the algorithm itself is time and storage consuming, yielding it infeasible for large-scale system. To overcome these problems, the EnKF was proposed, the specifics of which are introduced next.

There are many uncertain factors in groundwater modeling: initial and boundary conditions, forcing terms, parameter values, ... (Hendricks Franssen and Kinzelbach, 2009). In this paper, we focus on the uncertainty about the parameter log-hydraulic conductivity. The state-transition equation is the standard three-dimensional groundwater flow equation, which is solved by MODFLOW, one of the most popular three-dimensional finite-difference groundwater flow simulators (e.g., McDonald and Harbaugh, 1988; Harbaugh et al., 2000). The filter has two steps. The first one is the prediction step, given by

$$
s_{i}^{f}(t)=M\left(s_{i}^{a}(t-\Delta t)+w_{i}(t), w(t) \sim N(0, \sigma)\right.
$$

where $s_{i}^{f}(t)$ is the forecasted state of the system for a given set of parameters $i$, this state is function of the last estimate of the state of the system at $t-\Delta t, s_{i}^{a}(t-\Delta t)$ through a state-transition equation represented by $M$. Equation $M$ is only an approximation of how the system behaves, therefore 
a model error $w$ is added to the forecast, which is assumed to be Gaussian distributed, with zero mean and covariance $\sigma$.

The second step is the analysis step (Burgers et al., 1998), whereby the observed state measurements are used to update the forecast state to come up with a better estimate of the forecasted state.

$$
\begin{gathered}
s_{i}^{a}(t)=s_{i}^{f}(t)+k(t)\left[d_{o}(t)+e_{i}(t)-H s_{i}^{f}(t)\right] \\
k(t)=p^{f}(t) H^{T}\left[H p^{f}(t) H^{T}+R\right]^{-1}
\end{gathered}
$$

where the forecasted state $s_{i}^{f}(t)$ is updated as a function of the difference between the predicted state $s_{i}^{f}(t)$ and the observations $\left(d_{o}(t)+e_{i}(t)\right.$; matrix $H$ is a measurement matrix that serves to map the model predictions (generally over a fixed grid) onto the locations where the observations are taken. Note that the observations are composed of two terms the "real" state of the system $d_{o}(t)$ plus a measurement error $e_{i}(t)$ of mean zero and covariance $R$. The amount by which the forecasted state should be modified is controlled by the Kalman gain $k(t)$, which is a function of the state covariance $p^{f}(t)$. The ensemble Kalman filter was developed precisely to avoid the shortcomings in computing the state covariance by linearizing the statetransfer equation. For this purpose, an ensemble of realizations is generated, and their state is forecasted and updated sequentially in time as data are collected. At each time step, the covariance is numerically inferred from the ensemble of realizations,

$$
\begin{gathered}
p^{f}(t)=\frac{1}{N_{e}-1} \sum_{i=1}^{N_{e}}\left(s^{f}(t)-\left\langle s^{f}(t)\right\rangle\right)\left(s^{f}(t)-\left\langle s^{f}(t)\right\rangle\right)^{T} \\
\left\langle s^{f}(t)\right\rangle=\frac{1}{N_{e}} \sum_{i=1}^{N_{e}} s^{f}(t)
\end{gathered}
$$

where $N_{e}$ is the number of realizations of the ensemble, $\langle\cdot\rangle$ denotes ensemble average, and $p$ is a matrix of dimensions $n \times n$, with $n$ is the number of nodes at which the state of the system is predicted by the numerical forecast model.

In inverse modeling applications, the state of the system is augmented so that it not only includes the properly-speaking state of the system but also the parameters defining the transfer function. In hydrogeology it is common to use such an augmented state, in our case we use piezometric heads and log-hydraulic conductivities as state and parameters, respectively. In our implementation we use an augmented vector, the state transfer function in Eq. (2.1) leaves unchanged the logconductivity values, and updates the piezometric heads according to the groundwater flow model; then, in the analysis step, we limit ourselves to update the logconductivity values as explained next. For the sake of demonstration, we will assume that observations are taken at groundwater model prediction locations; limiting the 
component of the measurement matrix $H$ to be 0 's and 1's, and simplifying the expressions in Eqs. (2.2) and (2.3) as follows. We may partition the covariance matrix

$$
p=\left[\begin{array}{ll}
p_{h h} & p_{Y h} \\
p_{h Y} & p_{Y Y}
\end{array}\right]
$$

where $p_{h h}, p_{Y Y}$ are the covariance between hydraulic heads at measurement locations and the covariance between log-conductivities at all model gridnodes, respectively, and $p_{Y h}$ and $p_{h Y}$ are the cross-covariances between a log-conductivity and a hydraulic head. And Eqs. (2.2) and (2.3) become

$$
\begin{gathered}
Y_{i}^{a}(t)=Y_{i}^{f}(t)+k(t)\left[d_{o}(t)+e_{i}(t)-h_{i}^{f}(t)\right] \\
k(t)=p_{Y h}\left[p_{h h}+R\right]^{-1}
\end{gathered}
$$

where $Y_{i}^{a}(t)$ and $Y_{i}^{f}(t)$ are the elements of the augmented state vector corresponding to the logconductivities, and $h_{i}^{f}(t)$ is the predicted piezometric heads at measurement locations.

The output of the EnKF is an ensemble of realizations of hydraulic conductivity all of which are "conditioned" to the observation data. From this ensemble we can obtain average estimates and uncertainty estimates about the hydraulic conductivity, or we can post-process these fields to obtain optimal estimates, with their associated uncertainty, of response functions based on the ensemble, such as optimal pumping rates for the dewatering of a construction site. As more observational data are assimilated, the ensemble of hydraulic conductivities become more alike, and therefore the uncertainty associated is smaller, since there are less alternative realizations capable of reproducing the observed entire transient state information.

We end this introduction mentioned some of the main advantages and drawbacks of the EnKF. The main advantage of the EnKF is that is not an optimization approach but rather a filtering approach. Therefore, there is no need for recursive evaluations of expensive cost functions, just the need to compute the ensemble covariance and the mismatch between predictions and observations, which will be used to update the conductivity fields. This characteristic makes the EnKF very easy to implement and to apply in cases with many different sources of information. The main drawback, leaving apart the Monte-Carlo aspect of the method and the need to handle many realizations, is that the EnKF has been found to collapse underestimating the final uncertainty (that is, as the fields keep been updated, they tend to become more and more similar). There are two main reasons for this behavior, one is the appearance of spurious correlations between distant points due to the numerical nature of the covariance calculations, the other has to do with the number of realizations, if it is not large enough, the empirical covariance based on a reduced number of realizations tends to 
decrease as new updating steps are performed. The approaches to deal with these problems are covariance localization and covariance inflation.

\subsection{Parallelization approach}

There are mainly three kinds of parallel computer architectures, the first kind is based on shared memory, the second one is based on distributed memory, and the third one is a hybrid in between both of them. The first type corresponds to multi-core computers, the benefits are that the communication between the different ranks is very fast, and that it is very easy to share data among them. However, this type of architecture is limited by the total amount of memory available, and may be unable to address large models. The second type corresponds to sharing resources among a grid of computers, the grid could easily be enlarged attaching new computers to it, and therefore the limitation about the size of the model they can handle disappears, on the contrary, the communication between the different processors is much slower than in the shared-memory architecture. The best architecture is the third one that uses a grid of multi-core computers, balancing the advantages and disadvantages of the first and second architecture kinds.

In this paper, we employ a hybrid architecture. The cluster of computers, known as "Pleiades", consists of three HP Proliant DL 580, each with sixcore four processors AMD Opteron Model $8439 \mathrm{SE}$ (six-core, 2.8GHz, 6MB L3, 105W), which amounts to a total of 24 cores per computer. All cores are 64-bit, and each computer has 256 GB of RAM. The operating system is CentOS. Communication between processors is via message passing interface (MPI). The cluster is networked via Ethernet TCP/IP.

As already explained previously, the EnKF consists of two steps: forecast and analysis (or updating). We have analyzed the potential for parallelization of both steps.

Fig. 2.1 shows a flowchart of the proposed parallelization, which is explained next.

\subsubsection{Forecast step}

The most straightforward way to parallelize the forecast step in the EnKF is at the realization level (Chen and Zhang, 2006). See box 1 of Fig. 2.1, let $s\left(n \cdot N_{e}\right)$ be the state vector including all the ensemble members, where $n$ represents state-vector size and $N_{e}$ is the number of realizations of the ensemble; if there are $k$ processors, then the metric can be decomposed into sub-metrics $s\left(n \cdot m_{0}\right), s\left(n \cdot m_{1}\right), \ldots, s\left(n \cdot m_{k}\right)$, where $m_{0}, m_{1}, \ldots m_{k}\left(m_{0}+m_{1}+\ldots+m_{k}=N_{e}\right)$ denotes the number of ensemble members that must be processed by each processing element, $P E_{0}, P E_{1}, \ldots P E_{k}$ respectively. Once the realizations are distributed among the processors, the MODFLOW simulator is called 


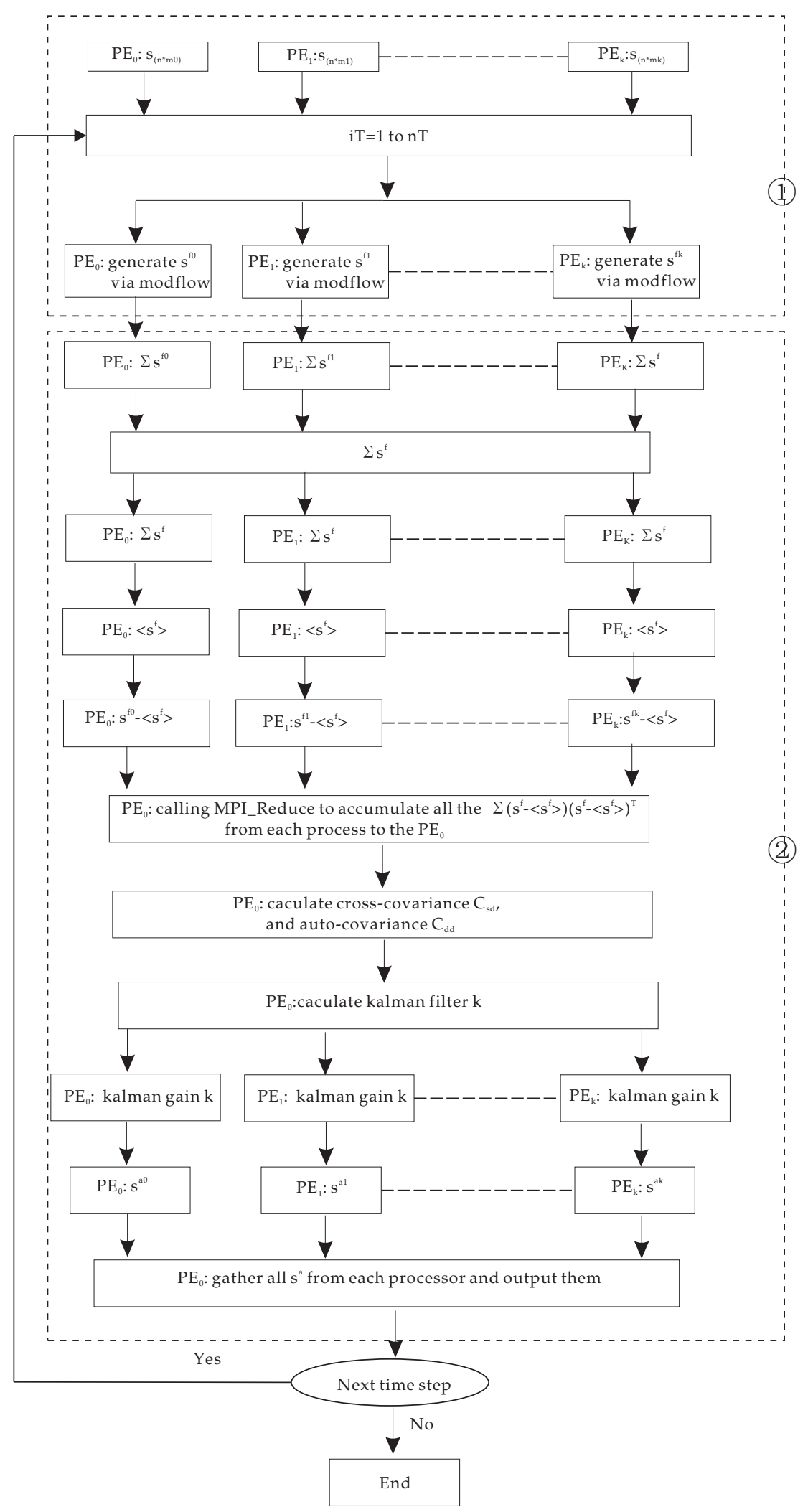

Figure 2.1. The parallel EnKF flow chart: box 1 is for the forecast step; box 2 is for the updating step. 
to generate the forecast piezometric heads in all realizations. The distribution of the realizations to the processors must be so that the load on all processors is as similar as possible. If $N_{e}$ is a multiple of $k$, then each processor will receive $N_{e} / k$ realizations, otherwise there will be $N_{e} \% k$ processors receiving one extra realization.

Since the processors operate asynchronously, and the analysis step cannot be performed until the forecast is performed in all realization, it is necessary to call the MPI_Barrier command before starting the update step, to block each processor until all processors reach the barrier point in the code (Dewaraja et al., 2002).

\subsubsection{Update step}

After the forecast step, the state vector $s^{f}$, which contains all realizations, is distributed between the processors. Next, we have to compute the covariances $p_{Y h}$, and $p_{h h}$. This calculation is distributed as follows, first accumulate the distributed state vector $s^{f}$ in each processor, $\sum s^{f 0}, \sum s^{f 1}, \ldots$, $\sum s^{f k}$, (recall that each processor is in charge of the forecast of a subset of all realizations) send these accumulated values to one of the processors $P E_{0}$ and compute the mean value of each component. Broadcast the mean values to all of the processors, and accumulate the products of the differences of the state vector with respect to their means in each one of the processors $\sum\left(s^{f 0}-<s^{f}>\right)\left(s^{f 0}-<s^{f}>\right)^{T}, \ldots, \sum\left(s^{f k}-<s^{f}>\right)\left(s^{f k}-<s^{f}>\right)^{T}$. Then, send the accumulated products of differences to $P E_{0}$ and compute the covariances in this processor. Notice that the accumulated products of differences must be computed only for the individual entries in $p_{Y h}$, and $p_{h h}$, not for all possible entries of $p^{f}$ in Eq. (2.6). With the covariances computed, the Kalman gain is obtained and broadcasted back to all processors so that the updating Eq. (2.7) is applied to each member of the state vector distributed between the processors.

\subsection{Application}

The 3-D transient groundwater flow equation is:

$$
S_{s} \frac{\partial h}{\partial t}-\nabla \cdot(k \nabla h)=W
$$

where $S_{s}$ is specific storage coefficient $\left[L^{-1}\right] ; h$ is the hydraulic head $[L] ; k$ is the hydraulic conductivity $\left[L T^{-1}\right] ; t$ is the time $[T] ; W$ denotes sinks and sources per unit volume $\left[T^{-1}\right]$.

We use four test cases corresponding to two different model sizes. Cases 1, 2 and 3 use a synthetic model with 50 by 50 by 5 cells, and the difference between them is the number of realizations in the ensemble, which are 1200 , 
720 and 240. Case 4 uses a synthetic model with 50 by 50 by 1 cell and 1200 realizations in the ensemble. All cells are $5 \mathrm{~m}$ by $5 \mathrm{~m}$ by $2 \mathrm{~m}$.

For the small model of size 50 by 50 by 1 cells, there are 75 observation wells in the domain located as shown in Fig. 2.2. The left boundary has a specified head boundary equal to $8 \mathrm{~m}$, the right boundary has a specified flow of $-0.0008 \mathrm{~d}^{-1}$ and the top and bottom boundaries are no flow.

For the large model of size of 50 by 50 by 5 cells, there are 25 observation wells in the domain, as shown in Fig. 2.3, that monitor the piezometric heads at the first, third and fifth layers, for a total of 75 measurements. A verification well, located at row 30, column 20 and layer 3 (see Fig. 2.3) is used to test the evolution of the piezometric head at an unsampled location. The first layer of the left boundary is given a specified head of $8 \mathrm{~m}$, the fifth layer of the right boundary is given a prescribed flow of $-0.008 \mathrm{~d}^{-1}$. The rest of the boundary are no flow boundaries.

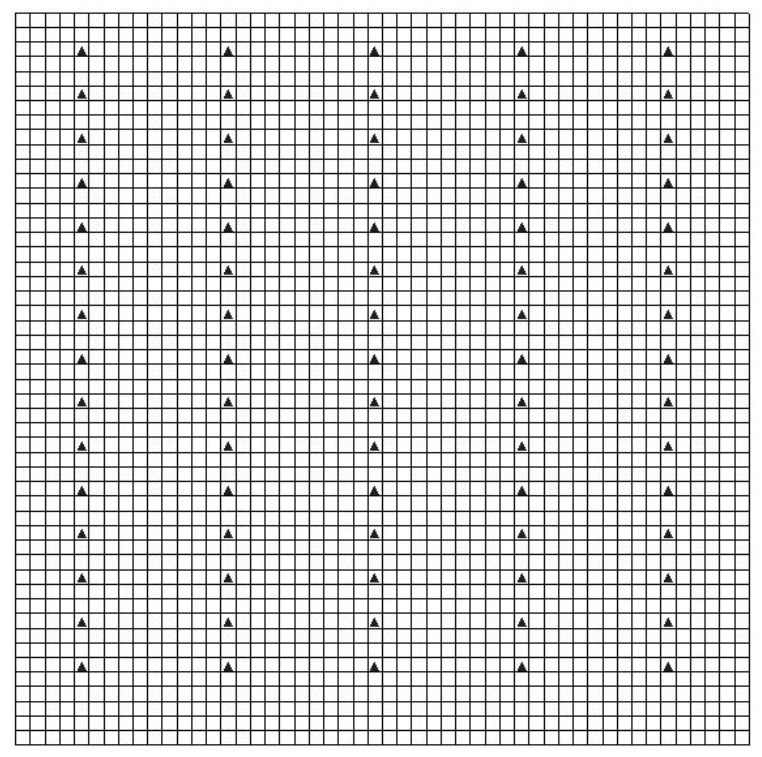

Figure 2.2. Flow domain and location of the 75 observation wells in the small model.

For both models, the initial head is set $8 \mathrm{~m}$ throughout the domain. Specific storage $S_{s}$ is set as $0.0008 \mathrm{~m}^{-1}$. The total simulation time is 500 days, discretized into 100 time steps according to the following progression

$$
\triangle t_{k}=\delta \triangle t_{k-1}
$$

where $\delta$ is 1.05 .

Log-conductivity $\ln (k)$ is assumed to be second-order stationary following a multi-Gaussian distribution of zero $\ln \mathrm{m} / \mathrm{d}$ mean, standard deviation $\sigma=1.5 \ln \mathrm{m} / \mathrm{d}$, and an exponential covariance function. 


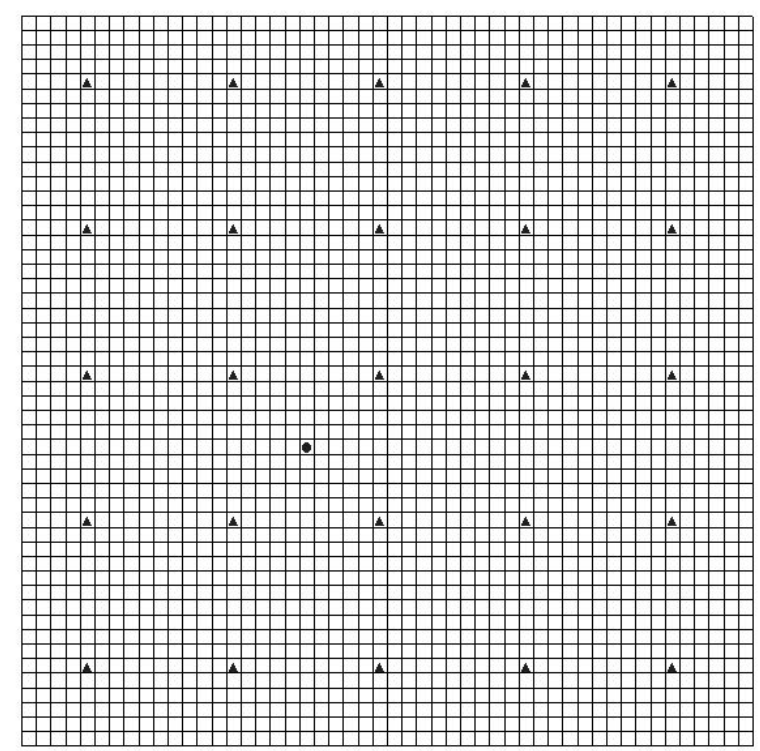

Figure 2.3. Flow domain and location of the 25 observation wells in the large model. The filled triangles are observation wells measuring the piezometric heads at the first, third and fifth layers, and the filled circle is a verification well.

$$
r(h)=\sigma^{2}\left[1-\exp \left(-\frac{\left|h_{x}\right|}{\lambda_{x}}-\frac{\left|h_{y}\right|}{\lambda_{y}}-\frac{\left|h_{z}\right|}{\lambda_{z}}\right)\right]
$$

where the integral scales in the $x, y, z$ directions are respectively $\lambda_{x}=90$ $\mathrm{m}, \lambda_{y}=30 \mathrm{~m}, \lambda_{z}=5 \mathrm{~m}$.

The sequential Gaussian simulation module of the GSLIB software (Deutsch and Journel, 1998a) is used to generate the log-conductivity realizations. One of the realizations is chosen as the reference field.

\subsubsection{Analysis}

Fig. 2.4 shows the reference log-conductivity field, and Fig. 2.5a,2.5b,2.5c,2.5d,2.5e,2.5f shows the ensemble mean field for the tests 1,2 and 3, at the beginning of the simulation (when no piezometric head data has been assimilated yet) and at the 50th time step. While comparing Fig.2.5b,2.5d,2.5f and Fig. 2.4, we find that the main features of the reference field are captured by the EnKF after the 50th data assimilation step. The larger the ensemble size, the smoother is the ensemble mean.

Fig. 2.6a, 2.6c and 2.6e shows the piezometric heads in the control well (not used for conditioning) computed on the initial ensemble of logconductivity realizations. They display a very large variability indicative of large prediction uncertainty. This variability is reduced when the piezometric 


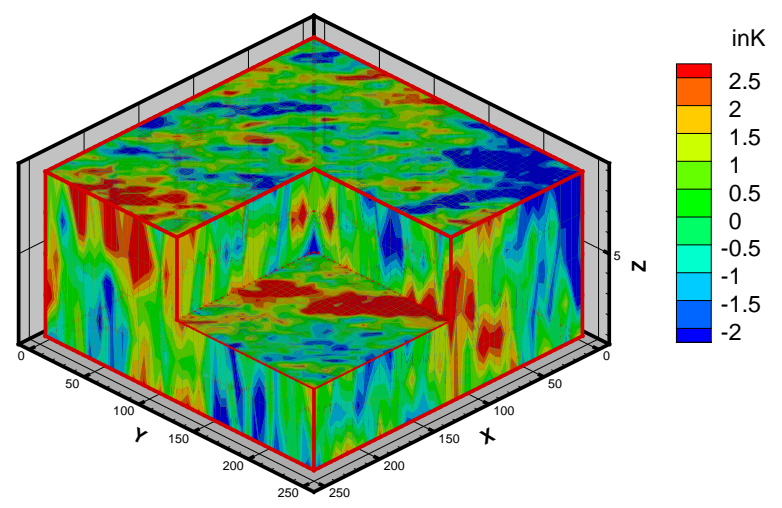

Figure 2.4. Reference realization of $\ln (k)$.

heads are computed on the updated realizations, as seen in Fig. 2.6b, 2.6d and $2.6 \mathrm{f}$.

We can evaluate the goodness of the estimated field using the average deviation between the average of the ensemble members and the reference field using the root mean square error (RMSE).

$$
R M S E=\sqrt{\frac{1}{N} \sum_{i=1}^{N}\left(s^{r e f}-\left\langle s^{a}\right\rangle\right)^{2}}
$$

Where $N$ is the number of model gridblocks; $s^{r e f}$ is the value of the reference field; $\left\langle s^{a}\right\rangle$ denotes the mean estimation of the ensemble fields.

Fig. 2.7 shows that the $R M S E$ of test 3 has some fluctuations at early assimilation steps, yet after the 31st assimilation step, it begins to increase, becoming even larger than the starting value, which implies that for a small ensemble size the estimation of the covariance is poor (Franssen and Kinzelbach, 2008). In the comparison of the RMSE between tests 1 and 2, the $R M S E$ of test 1 is lower, plus, it is gradually smoother after the 27th assimilation step. So it can be concluded that the larger the ensemble, the better the estimation.

Similar results and conclusions can be reached for fourth test case, for which the model size is smaller while retaining the same number of measurements.

\subsubsection{Parallelization analysis}

Speedup and efficiency are usually used to evaluate the performance of parallel algorithms 

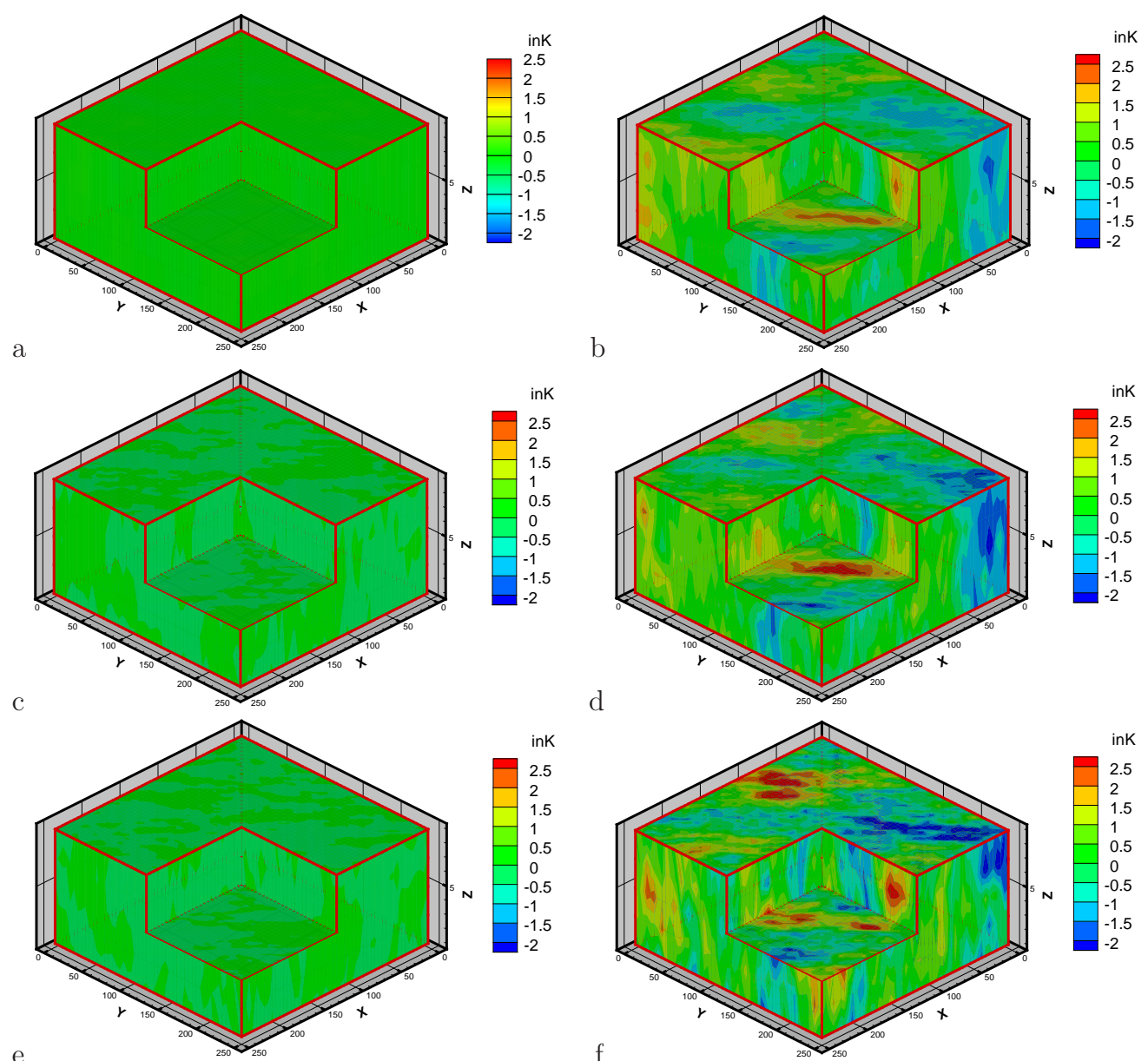

Figure 2.5. initial and updated ensemble means facies fields.(a), (b) show the ensemble mean facies fields of the initial realizations and the 50th updated realizations of test 1; (c), (d) show the ensemble mean facies fields of the initial realizations and the 50th updated realizations for test 2 ; (e), (f) show the ensemble mean facies fields of the initial realizations and the 50th updated realizations for test 3 . 

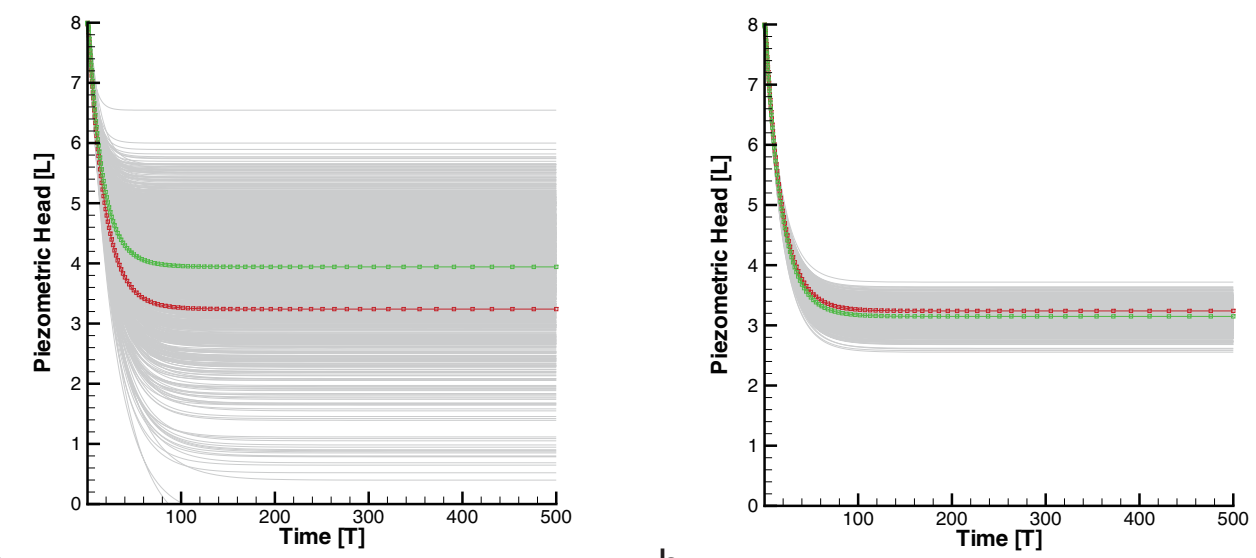

a

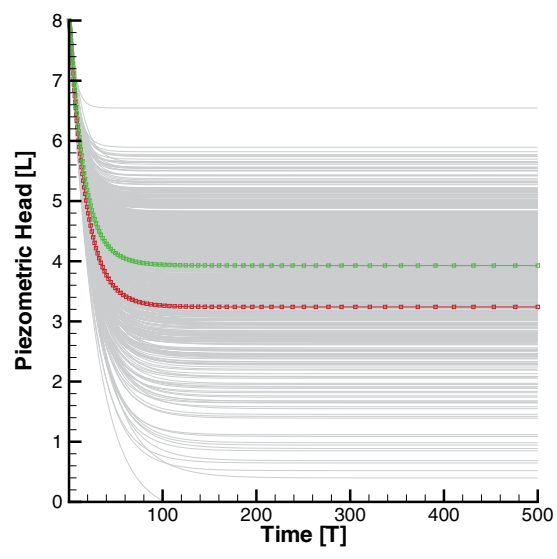

C

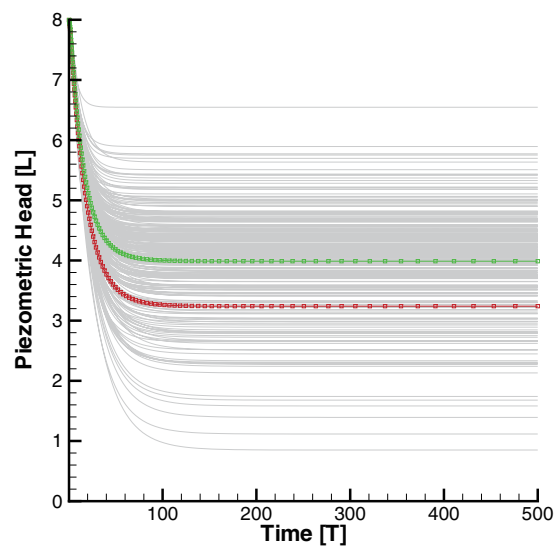

b

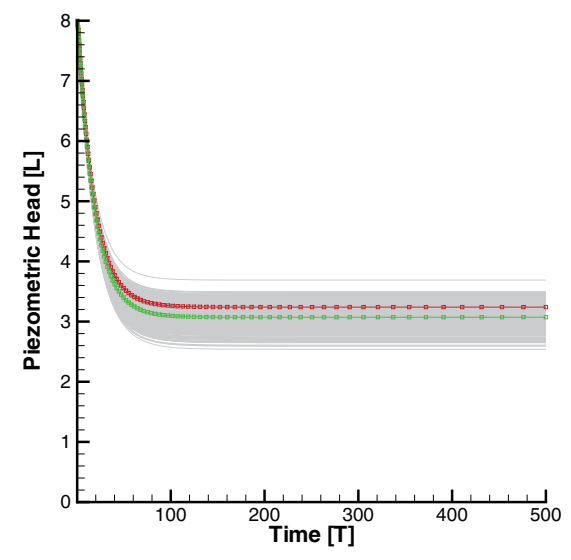

d

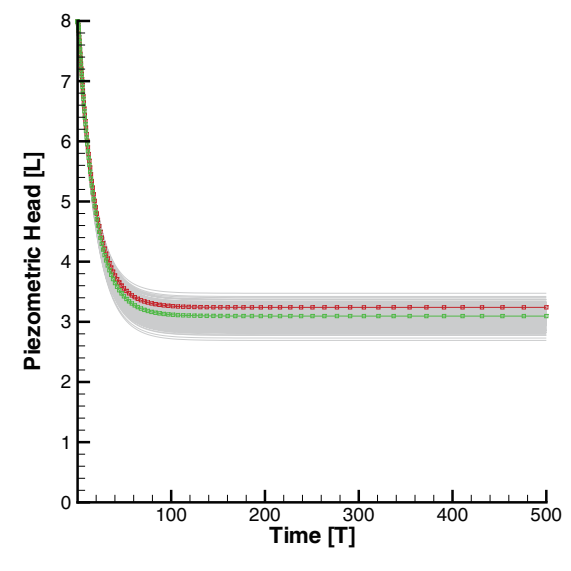

Figure 2.6. Piezometric head time evolution at the control well. The red curve is for the reference field, the gray curves for each realization of the ensemble, and the green curve is the ensemble mean. (a) and (b) show piezometric heads of the the initial realizations and the 50th updated realizations for test 1; (c) and (d) show piezometric heads of the initial realizations and the 50th updated realizations for test 2; (e) and (f) show piezometric heads of the initial realizations and the 50th updated realizations for test 3 . 


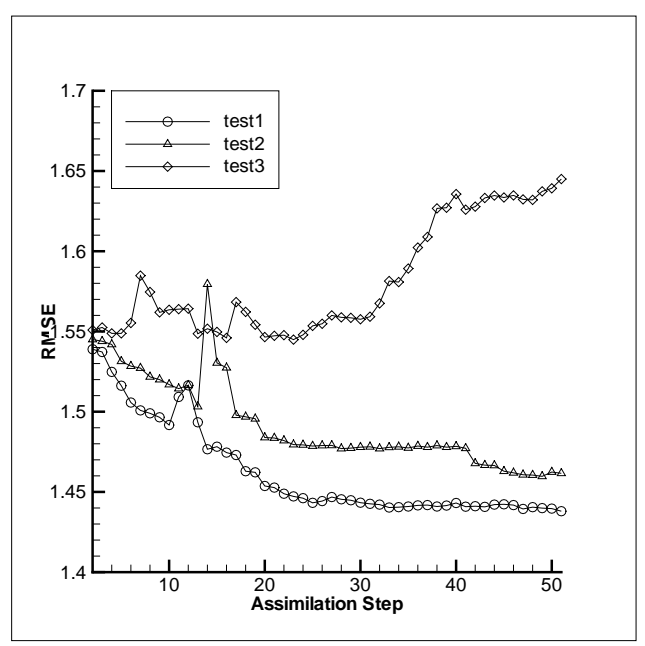

Figure 2.7. The root mean square error for three test.

$$
\begin{gathered}
\text { Speedup: } S_{P}=\frac{T_{s}}{T_{P}} \\
\text { Efficiency: } E_{P}=\frac{S_{P}}{P}
\end{gathered}
$$

$P$ is the number of the processors, $T_{s}$ is the CPU time consumed under a serial implementation of the algorithm, $T_{P}$ is the CPU time consumed under a parallel implementation with $P$ processors.

There is an obvious trade-off between the ensemble size and the CPU time, and also between the size of the numerical model and the CPU time. Table 2.1 and Fig. 2.8 show the performance of the parallel algorithm for test cases 1 (three-dimensional domain and 1200 realizations), 3 (threedimensional domain and 240 realizations) and 4 (two-dimensional domain and 1200 realizations ).

From the table and figure we can see that the speedup is far from the ideal line for which the improvement in CPU should follow the same proportion as the number of processors. This is not a surprising result, there are two causes for this departure from the ideal performance: there is a need for all processors to wait until they have finished certain tasks for them to proceed on to the next task, and there is extra time needed for communication between processors.

We notice some differences between the tests. Comparing tests 1 and 3 , we notice that the speedup is better for the case with the larger number of realizations, this is because for test 3 , each processor receives a smaller number of realizations and thus, proportionally, the time spent in communication is larger for test 3 than for test 1 . Apparently, for both tests, the 

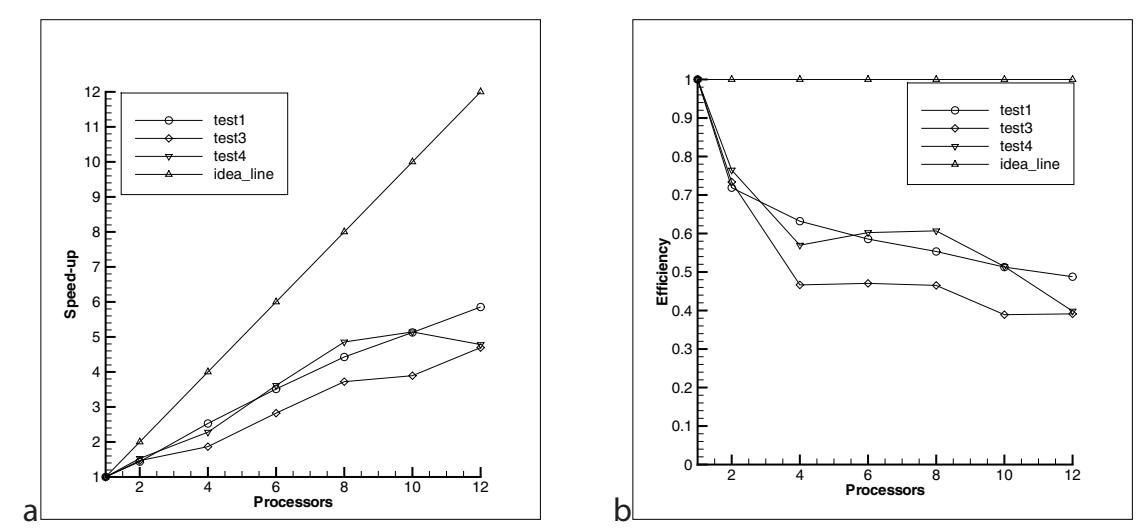

Figure 2.8. Parallel speed-up and efficiency. (a) Speed up (b) efficiency.

Table 2.1. Parallel performance of three of the tests.

\begin{tabular}{|c|c|c|c|c|c|c|}
\hline Processors & Test1CPUs & Speedup & Efficieny & Test3CPUs & Speedup & Efficieny \\
\hline 1 & 20539.92 & 1.00 & 1.00 & 4866.50 & 1.00 & 1.00 \\
\hline 2 & 14284.37 & 1.44 & 0.72 & 3316.35 & 1.47 & 0.73 \\
\hline 4 & 8125.83 & 2.53 & 0.63 & 2607.83 & 1.87 & 0.47 \\
\hline 6 & 5846.27 & 3.51 & 0.59 & 1723.75 & 2.82 & 0.47 \\
\hline 8 & 4640.38 & 4.43 & 0.55 & 1307.34 & 3.72 & 0.47 \\
\hline 10 & 4005.09 & 5.13 & 0.51 & 1250.23 & 3.89 & 0.39 \\
\hline 12 & 3508.46 & 5.85 & 0.49 & 1036.38 & 4.70 & 0.39 \\
\hline Processors & Test4CPUs & Speedup & Efficieny & & & \\
\hline 1 & 10087.23 & 1.00 & 1.00 & & & \\
\hline 2 & 6595.59 & 1.53 & 0.76 & & & \\
\hline 4 & 4426.79 & 2.28 & 0.57 & & & \\
\hline 6 & 2790.85 & 3.61 & 0.60 & & & \\
\hline 8 & 2077.76 & 4.85 & 0.61 & & & \\
\hline 10 & 1961.45 & 5.14 & 0.51 & & & \\
\hline 12 & 2109.46 & 4.78 & 0.40 & & & \\
\hline
\end{tabular}


speedup could keep increasing if more processor were available. For test 4, however, the speedup is similar to that of test 1 up to eight processors, then it appears to worsen, again, the cause is found in the increase in the communication time among processors. It does not seem that increasing the number of processors past 8 will increase the speedup (although the overall CPU time will be still reduced) for test 4 .

The efficiency curves have a similar behavior as the speedup curves. Efficiency worsens past 8 processors for test 4 , and for tests 1 and 3 we can conclude that the parallel algorithm is more efficient the larger the ensemble size.

We can conclude that the parallel implementation of the EnKF runs with higher efficiency for large size models and large ensembles than for small ones. In all cases, the final CPU time is smaller than for the serial implementation, although the speedup is still far from ideal.

In this parallel algorithm there are two inherent barriers to its processing capabilities, one is data asynchrony, and the other is data-dependency. Data asynchrony makes all processors be as slow as the slowest one, since they have to wait for all processors to finish a certain task before they can proceed to the next one. Data dependency refers to the fact that the distribution of realizations to the processors does not necessarily leaves all processors with the same number of realizations. Data asynchrony mainly affects the forecast step, while data dependency affects all steps and it is less noticed for large number of realizations.

\subsection{Summary and suggestion}

A parallel algorithm for the forecast and analysis steps of the EnKF has been presented that reduces significantly the time to run the EnKF for large ensemble sizes. The efficiency remains over 0.40 when using up to 12 processors for the two tests using 1200 realizations.

There are many measures that could be taken to reduce the communication time and increase the efficiency such as decreasing the communication traffic, boosting communication granularity or using a high-speed internet protocol to reduce the information transfer delay. In addition, we must attempt to keep the load as balanced as possible among the processors, that is, all processors should work on the same (or very similar) number or realizations.

Furthermore, the algorithm could be improved using a parallelized version of the groundwater flow simulator, such as one employing domain decomposition, especially for large size models. 


\title{
The Power of Transient Piezometric Head Data in Inverse Modeling: An Application of the Localized Normal-score EnKF with Covariance Inflation in a Heterogenous Bimodal Hydraulic Conductivity Field
}

\begin{abstract}
The localized normal-score ensemble Kalman filter (NS-EnKF) coupled with covariance inflation is used to characterize the spatial variability of a channelized bimodal hydraulic conductivity field, for which the only existing prior information about conductivity is its univariate marginal distribution. We demonstrate that we can retrieve the main patterns of the reference field by assimilating a sufficient number of piezometric observations using the NSEnKF. The possibility of characterizing the conductivity spatial variability
\end{abstract}


using only piezometric head data shows the importance of accounting for these data in inverse modeling.

\subsection{Introduction}

It is well known that proper characterization of subsurface hydrogeologic properties and their uncertainty are critical issues for groundwater forecast, subsurface resource management and environmental risk assessment ( $\mathrm{Li}$ et al., 2011). This can be achieved by stochastic inverse modeling accounting for real-time state data. Some existing methods for stochastic inverse modeling are the gradual deformation method, the sequential self-calibration, the Markov chain Monte Carlo method, the Representer method, and the Pilot Points method (e.g., Capilla and Llopis-Albert, 2009; Hu, 2000; GómezHernánez et al., 1997; Fu and Jaime Gómez-Hernández, 2009; Oliver et al., 1997; Alcolea et al., 2006; Wen et al., 2002; RamaRao et al., 1995; Franssen et al., 2003). Although these methods are flexible with regard to nonlinearities and system complexity, they are very time consuming and not easy to apply to large scale problems (Franssen and Kinzelbach, 2008).

To overcome this problem, the Ensemble Kalman Filter (EnKF) has become more popular in many fields, such as oceanography, meteorology, petroleum engineering, or hydrology (e.g., Aanonsen et al., 2009; Chen and Zhang, 2006; Evensen, 2003; Moradkhani et al., 2005; Dowell et al., 2004; Bertino et al., 2003a; Franssen and Kinzelbach, 2008; Li et al., 2012a), because it is computationally efficient and capable of handling large fields. However, it has been shown that, although the EnKF is good accounting for the non linearities of the state equation, it fails when dealing with nonGaussian parameter fields (e.g., Sun et al., 2009; Simon and Bertino, 2009; Chen et al., 2009).

The particle filter (PF) (e.g., Gordon et al., 1993; Losa et al., 2003; Van Leeuwen, 2009) is able to handle any type of statistical distribution and it is very robust for nonlinear models and non-Gaussian distributed variables (Schöniger et al., 2012); however, it is also very time-consuming and hardly applied to large simulation models.

Recently, new methods have been developed trying to adapt the EnKF to non-Gaussian distributions. They can be grouped in four categories according to their characteristics: those using a Gaussian mixture model (GMM), those using a transformed reparameterization, the iterative EnKF, and those using a Gaussian anamorphosis (GA) also known as normal score (NS) transform.

In the first category, the methods using a Gaussian mixture model method apply a probabilistic model in which a finite number of Gaussian probability density functions (pdf's) is used to approximate the underlying nonGaussian pdf's (e.g., Apte et al., 2007; Sun et al., 2009; Chen and Liu, 2000; 
Dovera and Della Rossa, 2011; Reich, 2011; Stordal et al., 2011). GMM takes advantage that for linear transfer functions the forecasting step preserves the Gaussian mixture. Sun et al. (2009) showed the benefits of the EnKF integrated with GMM techniques for high-dimensional, multimodal parameter distributions. Dovera and Della Rossa (2011) combined the EnKF with GMM for simulating a multimodal distribution in the context of reservoir facies modeling.

In the second category, the transformed reparameterization, the methods work with alternative state variables that may be better approximated by a Gaussian distribution. Chen and Oliver (2010b) discuss that using the EnKF to update saturation may yield non-physical results because of its nonGaussian distribution. They proposed to reparameterize the formulation of the EnKF using the water arrival time as the state variable. This approach has also been followed by Chen et al. (2009); Chang et al. (2010); Li et al. (2007).

In the third category, the iterative EnKF, an iterative scheme is introduced into the forecasting and updating steps of the EnKF. At any given time step, the static parameters are repeatedly updated using the Kalman gain equation until a satisfactory match between predicted state variables and observations is reached. This iteration is needed because of the strong non-linearities of the forecast model. Example applications can be found in the petroleum engineering literatures (e.g., Liu and Oliver, 2005; Gu and Oliver, 2007, 2006; Wen and Chen, 2005, 2006; Li and Reynolds, 2007; Wang et al., 2010; Zhao et al., 2008), and also in hydrogeology (Franssen and Kinzelbach, 2008).

In the fourth category, the EnKF is combined with GA. Gaussian anamorphosis (also known as normal score transform) is used to transform the non-Gaussian variables into Gaussian ones, but only at the univariate level. Then the EnKF is used on the univariate Gaussian variables. Applications of this approach can be found in the fields of ecology, remote sensing, geophysics, petroleum engineering or hydrogeology (e.g., Simon and Bertino, 2009, 2012; Bertino et al., 2003a,b; Bocquet et al., 2010; Béal et al., 2010; Schöniger et al., 2012; Zhou et al., 2011; Li et al., 2012b).

In this paper, we will apply the GA implementation by Zhou et al. (2011) to a bimodal aquifer assuming that the only information we have about the hydraulic conductivity is its univariate distribution. Our conjecture is that the assimilation of enough transient piezometric head data is sufficient to capture the main features of the spatial variability of hydraulic conductivity.

The structure of this paper is as follows. First, an introduction of the GA implementation is given. And then we evaluate the impact of the number of conditioning piezometric heads in the characterization of the conductivity patterns. The paper ends with a summary of the main findings. 


\subsection{The Localized Normal-Score Ensemble Kalman Filter with Covariance Inflation}

The Normal-Score Ensemble Kalman Filter (NS-EnKF) is an evolution of the EnKF to accommodate non-Gaussian random variables. It is based on a univariate transformation of each component of the parameter vector of non-Gaussian conductivities into another vector in which all components follow a standard Gaussian distribution.

We will present the NS-EnKF for the case in which we wish to characterize the heterogeneity of hydraulic conductivity $(X)$ by assimilating transient piezometric heads $(Y)$. The NS-EnKF can be summarized as follows:

1. Initialization step. An ensemble of hydraulic conductivity fields must be generated. There are many techniques that can be used for this purpose, such as sequential simulation, multiple point simulations with training images (e.g., Gómez-Hernández and Journel, 1993; Strebelle, 2002; Mariethoz et al., 2010); however, since we assume that there is no prior information about the spatial heterogeneity, but only information about its marginal univariate distribution, we generate homogeneous realizations, each one with a value drawn from this distribution.

2. Normal-score transformation step. At each location, all conductivity values from all realizations are collected, and a normal score transform function is built. Then, these functions are used to transform all values for all realizations.

The normal score transformed conductivity vector $\widetilde{\mathbf{X}}$ is

$$
\widetilde{\mathbf{X}}=\phi(\mathbf{X})
$$

where $\phi(\cdot)$ is a vectorial normal score transform function, different for each location. Each member of the vectorial function is nonparametrically built.

3. Forecasting step. In this step, the simulated piezometric heads are calculated for the $t^{\text {th }}$ time step based on the piezometric heads from the $(t-1)^{t h}$ time step using a transient flow model, realization by realization.

$$
\mathbf{Y}_{t}=\psi\left(\mathbf{Y}_{t-1}, \mathbf{X}_{t-1}\right)
$$

where $\mathbf{Y}_{t}, \mathbf{Y}_{t-1}$ are the simulated piezometric heads at the $t^{t h}$ time step and the simulated piezometric heads at the $(t-1)^{t h}$ time step, respectively; $\mathbf{X}_{t-1}$ is the conductivity estimate at the $(t-1)^{t h}$ time step; $\psi(\cdot)$ denotes the transient groundwater flow model. 
4. Analysis step or assimilation step. The aim of this step is to update the transformed conductivity $\widetilde{\mathbf{X}}$ and piezometric heads $\mathbf{Y}$ accounting for the discrepancy between forecasted and observed piezometric heads.

(a) First, build the augmented state vector $\mathbf{S}$ with the transformed conductivity $\widetilde{\mathbf{X}}$ and the forecasted piezometric heads $\mathbf{Y}$, which for the $i^{t h}$ realization at the $t^{t h}$ time step is:

$$
\mathbf{S}_{i, t}^{f}=\left[\begin{array}{c}
\widetilde{\mathbf{X}} \\
\mathbf{Y}
\end{array}\right]_{i, t},
$$

(b) Then, the measured piezometric heads at time $t$ are assimilated by updating the state vector into $\mathbf{S}_{i, t}^{a}$ using:

$$
\begin{gathered}
\mathbf{S}_{i, t}^{a}=\mathbf{S}_{i, t}^{f}+\mathbf{G}_{t}\left(\mathbf{Y}_{t}^{o}+e_{i, t}-\mathbf{H} \mathbf{S}_{i, t}^{f}\right) \\
\mathbf{G}_{t}=\mathbf{P}_{t} \mathbf{H}^{T}\left(\mathbf{H} \mathbf{P}_{t} \mathbf{H}^{T}+\mathbf{R}_{t}\right)^{T}
\end{gathered}
$$

where $\mathbf{S}_{i, t}^{a}$ is the updated state vector of the $i^{\text {th }}$ ensemble member at the $t^{\text {th }}$ time step; $\mathbf{S}_{i, t}^{f}$ is the forecast state vector; $\mathbf{P}_{t}$ is the forecast covariance matrix; $\mathbf{Y}_{t}^{o}+e_{i, t}$ is the hydraulic head observation vector, including the true head $\mathbf{Y}_{t}^{o}$ plus the observation error $e_{i, t}$ - the observation error has with mean zero and covariance $\mathbf{R}_{t} ; \mathbf{G}_{t}$ is the Kalman gain; and $\mathbf{H}$ is an observation matrix, which consists only of 0 's and 1's when observations are taken at simulation nodes, in which case, Equation 3.5 can be simplified as

$$
\mathbf{G}_{t}=\mathbf{C}_{\widetilde{X} Y}\left(\mathbf{C}_{Y Y}+\mathbf{R}_{t}\right)^{-1}
$$

where $\mathbf{C}_{\tilde{X} Y}$ corresponds to the cross-covariance between the transformed state vector and the forecasted piezometric heads at the observation locations; and $\mathbf{C}_{Y Y}$ is the covariance between the forecasted piezometric heads at the observation locations.

5. Back transformation step. Back transform the updated normal-score transformed conductivities into conductivities using the inverse of the previously computed transform functions:

$$
\mathbf{X}=\phi^{-1}(\widetilde{\mathbf{X}})
$$

since a non-parametric transformation is used, there is a need to specify how to backtransform the values that are outside the range given by the minimum and maximum values used to build the non-parametric 
transform function. In our case we used the same approach as described in the Gslib library (Deutsch and Journel, 1998b) choosing a power interpolation with absolute bounds set at -4 and $4 \ln (\mathrm{m} / \mathrm{d})$.

6. Return to the step 3 and repeat the processes until all the observed data are assimilated.

Zhou et al. (2011, 2012) have shown that the NS-EnKF is a good alternative in the characterization of non-Gaussian distributed conductivity fields. However, since the NS-EnKF is based in the EnKF, it has the same drawbacks, that is, the appearance of spurious correlations between distant points and the underestimation of the final uncertainty. Spurious correlations appear due to the numerical nature of the covariance calculations, which result in fluctuating covariance estimates about zero at distances for which it should be zero. Underestimation of the final uncertainty is due to the underestimation of the empirical covariance based in a small number of realizations (Xu et al., 2012). These two problems can be tackled through combining covariance localizations and covariance inflation techniques.

Covariance localization aims to eliminate the effect of spurious correlations among the state variables and the parameters by constraining the correlation range of the empirical covariance. This can be achieved by replacing Equation 3.6 with the following equation:

$$
\mathbf{G}_{t}=\rho_{\tilde{X} Y} \circ \mathbf{C}_{\tilde{X} Y}\left(\rho_{Y Y} \circ \mathbf{C}_{Y Y}+\mathbf{R}_{t}\right)^{-1}
$$

where $\circ$ represents the Schur product; and $\rho_{\widetilde{X} Y}$ and $\rho_{Y Y}$ are localization functions used to correct $\mathbf{C}_{\tilde{X} Y}$ and $\mathbf{C}_{Y Y}$, respectively.

There are many alternatives to calculate the localization functions (e.g., Chen and Oliver, 2010a; Greybush et al., 2011; Houtekamer and Mitchell, 2001; Bergemann and Reich, 2010; Nan and Wu, 2011). In this paper, we use the same fifth-order distance dependent localization function (e.g., Gaspari, 1999; Hamill et al., 2001) for both covariances.

$\rho_{\widetilde{X} Y}(d)=\rho_{Y Y}(d)=\left\{\begin{array}{lc}-\frac{1}{4}\left(\frac{d}{a}\right)^{5}+\frac{1}{2}\left(\frac{d}{a}\right)^{4}+\frac{5}{8}\left(\frac{d}{a}\right)^{3}-\frac{5}{3}\left(\frac{d}{a}\right)^{2}+1, & 0 \leq \mathrm{d} \leq \mathrm{a} ; \\ \frac{1}{12}\left(\frac{d}{a}\right)^{5}-\frac{1}{2}\left(\frac{d}{a}\right)^{4}+\frac{5}{8}\left(\frac{d}{a}\right)^{3}+\frac{5}{3}\left(\frac{d}{a}\right)^{2}-5\left(\frac{d}{a}\right)+4-\frac{2}{3}\left(\frac{d}{a}\right)^{-1}, & \mathrm{a} \leq \mathrm{d} \leq 2 \mathrm{a} ; \\ 0 & \mathrm{~d}>2 \mathrm{a} .\end{array}\right.$

where $d$ is the Euclidean distance, and $a$ is a distance parameter controlling the distance at which the localization function will die out to zero. We chose this function based on our past experience (Li et al., 2012a,b).

Covariance inflation is a technique used to avoid filter divergence (inbreeding) by inflating the empirical covariance. This can be achieved by linearly inflating each component of the augmented state vector: 


$$
\mathbf{S}_{i, t}^{i n f, f}=\sqrt{\lambda_{t}}\left(\mathbf{S}_{i, t}^{f}-\left\langle\mathbf{S}_{t}^{f}\right\rangle\right)+\left\langle\mathbf{S}_{t}^{f}\right\rangle
$$

where $\mathbf{S}_{i, t}^{i n f, f}$ is the $i^{t h}$ ensemble member at the $t^{t h}$ time step of the state vector; $\langle\cdot\rangle$ denotes ensemble average; $\lambda_{t}$ is the inflation factor at the $t^{t h}$ time step. There are many methods to get the inflation factor $\lambda$ (e.g., Anderson, 2007a; Zheng, 2009; Liang et al., 2011; Li et al., 2009; Kurtz et al., 2011). In this work, we will use the time-dependent inflation algorithm proposed by Wang and Bishop (2003).

$$
\lambda_{t}=\frac{\left(\mathbf{R}_{t}^{-\frac{1}{2}} d_{t}\right)^{T} \mathbf{R}_{t}^{-\frac{1}{2}} d_{t}-k_{t}}{\operatorname{trace}\left\{\mathbf{R}_{t}^{-\frac{1}{2}} \mathbf{H} \mathbf{P}_{t}\left(\mathbf{R}_{t}^{-\frac{1}{2}} \mathbf{H}\right)^{T}\right\}}
$$

where $k_{t}$ is the number of observations; $d_{t}$ is the residual between observation data and forecast data, which can be described as:

$$
d_{t} \equiv \mathbf{Y}_{t}^{o}+e_{i, t}-\mathbf{H}\left\langle\mathbf{S}_{t}^{f}\right\rangle
$$

Then the transformed analysis state vector $\mathbf{S}_{i, t}^{a}$ is:

$$
\mathbf{S}_{i, t}^{a}=\mathbf{S}_{i, t}^{i n f, f}+\lambda_{t} \mathbf{C}_{\widetilde{X} Y}\left(\lambda_{t} \mathbf{C}_{Y Y}+\mathbf{R}_{t}\right)^{T}\left(\mathbf{Y}_{t}^{o}+e_{i, t}-\mathbf{Y}_{i, t}^{i n f, f}\right)
$$

where $\mathbf{Y}_{i, t}^{\text {inf,f }}$ contains the forecasted piezometric heads after inflation at the observation locations.

\subsection{Synthetic Example}

A synthetic bimodal confined aquifer consisting of $30 \%$ high permeability sand and $70 \%$ low permeability shale is constructed on a grid of 100 by 80 by 1 cells, each cell being $3 \mathrm{~m}$ by $3 \mathrm{~m}$ by $10 \mathrm{~m}$. The SNESIM code, a multiple-point simulation program developed by Strébelle (2000), is used to generate a two-facies field using the training image in Strebelle (2002) (see Figure 3.1). Then, the facies field is populated, independently for each facies, with log-conductivity values using a sequential Gaussian simulation algorithm (Gómez-Hernández and Journel, 1993). The parameters used in the sequential Gaussian simulations are shown in Table 3.1. The resulting reference log-conductivity field and its histogram are shown in Figures 3.2 and 3.3, respectively. We can see in Figure 3.2 that the distribution of logconductivities is clearly non-Gaussian, and that the field has well-connected sand channels. The bimodal distribution in Figure 3.3 has a global mean of $-0.3 \ln (\mathrm{m} / \mathrm{d})$, and a global standard deviation of $1.7 \ln (\mathrm{m} / \mathrm{d})$.

The boundary conditions used in the simulation of transient groundwater flow are: north and south boundaries, no flow; east boundary, prescribed 
Table 3.1. Parameters of the random functions describing the spatial continuity of the sand and shale logconductivities

\begin{tabular}{lccccccc}
\hline Facies & Proportion & $\begin{array}{c}\text { Mean } \\
{[\ln [\mathrm{m} / \mathrm{d}]]}\end{array}$ & $\begin{array}{c}\text { Std.dev } \\
{[\mathrm{ln}[\mathrm{m} / \mathrm{d}]]}\end{array}$ & $\begin{array}{c}\text { Variogram } \\
\text { type }\end{array}$ & $\begin{array}{c}\lambda_{x} \\
{[\mathrm{~m}]}\end{array}$ & $\begin{array}{c}\lambda_{y} \\
{[\mathrm{~m}]}\end{array}$ & sill \\
\hline Sand & 0.3 & 2.1 & 0.7 & exponential & 144 & 72 & 1 \\
Shale & 0.7 & -1.4 & 0.7 & exponential & 72 & 72 & 0.35 \\
\hline
\end{tabular}

flow as indicated in Figure 3.2; and west boundary, general head boundary condition with head at $2 \mathrm{~m}$ and leakage coefficient of $0.14 \mathrm{~d}^{-1}$. The initial head is set to zero throughout the domain. Specific storage is set to 0.003 $\mathrm{m}^{-1}$. The total simulation time is 500 days and it is discretized into 100 time steps. The time steps increase in size as time progresses following a geometric series with ratio 1.05. The transient flow simulator MODFLOW (e.g., McDonald and Harbaugh, 1984; Harbaugh et al., 2000) is used as the forward model.

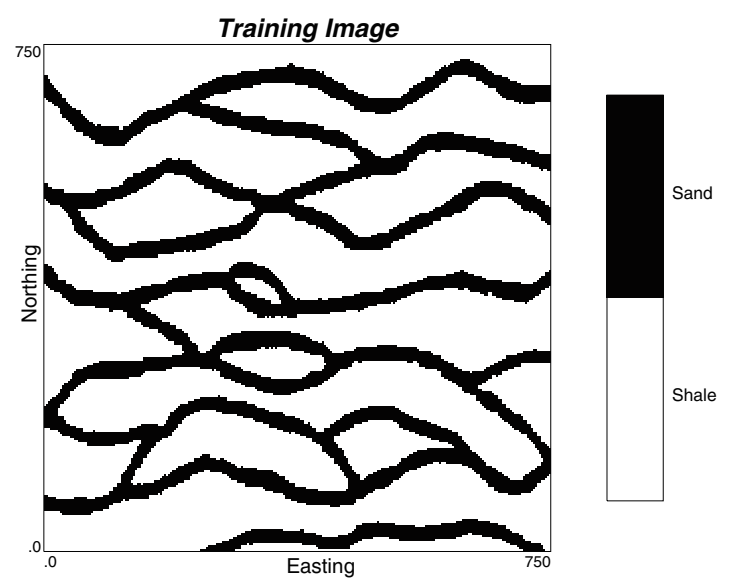

Figure 3.1. Training image used to generate the ensemble of binary facies realizations.

\subsubsection{Scenarios}

In this work, seven scenarios are used to demonstrate the power of transient piezometric head in the characterization of a bimodal hydraulic conductivity field. The impact of the covariance inflation in the characterization of the hydraulic conductivity field (see Table 3.2) is also analyzed. It is important to recall that no prior information about the spatial variability of conductiv- 


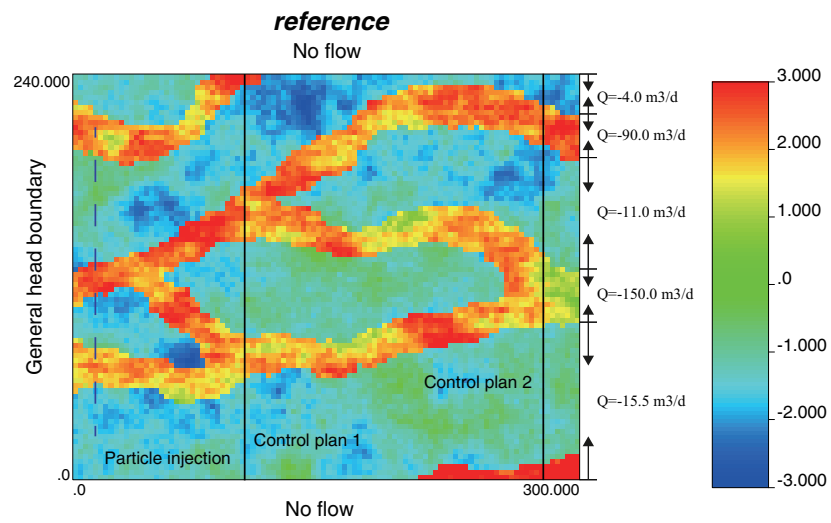

Figure 3.2. Reference field. The line source where particles are injected is shown with the blue dashed line, the two control planes indicated by the black lines are used to compute breakthrough curves.

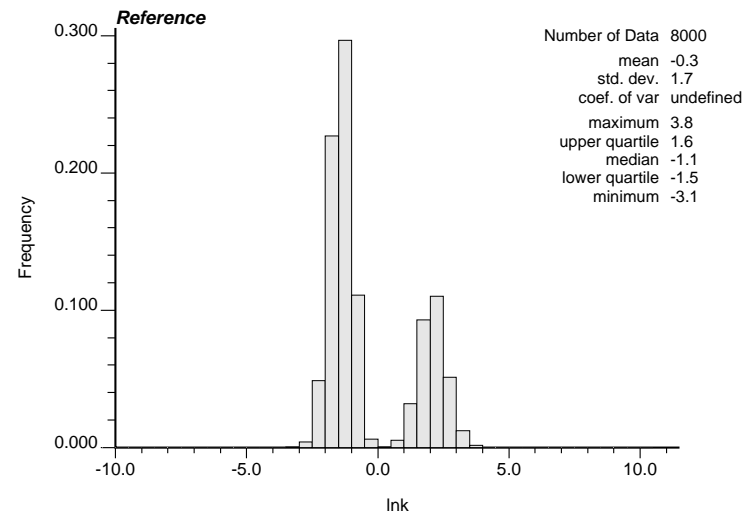

Figure 3.3. The histogram of the reference field 
Table 3.2. Definition of scenarios

\begin{tabular}{|c|c|c|c|c|c|c|c|}
\hline Scenario & S0 & S1 & $\mathrm{S} 2$ & S3 & $\mathrm{S} 4$ & S5 & S6 \\
\hline Initial Homogenous fields & & $\sqrt{ }$ & 1 & $\sqrt{ }$ & $\sqrt{ }$ & $\sqrt{ }$ & $\sqrt{ }$ \\
\hline Initial Heterogenous fields & $\sqrt{ }$ & & & & & & \\
\hline Observation piezometers (111) & & $\sqrt{ }$ & $\sqrt{ }$ & & & & \\
\hline Observation piezometers (56) & $\sqrt{ }$ & & & $\sqrt{ }$ & $\sqrt{ }$ & & \\
\hline Observation piezometers (24) & & & & & & $\sqrt{ }$ & \\
\hline No variance inflation & $\sqrt{ }$ & $\sqrt{ }$ & & $\sqrt{ }$ & & $\sqrt{ }$ & \\
\hline Variance inflation & & & $\sqrt{ }$ & & $\sqrt{ }$ & & $\sqrt{ }$ \\
\hline
\end{tabular}

ity is used, and that no conditioning hydraulic conductivity data are used, either.

For reference purposes, we include a Scenario S0 in the analysis. This scenario replicates the analysis performed by Zhou et al. (2011), where they had information about the spatial variability of hydraulic conductivity in the form of the training images from which the reference case had been generated; therefore, the training image of Figure 3.1 is used to generate 1000 unconditional realizations of the two-facies distribution, which are later populated with conductivity values by Gaussian sequential simulation, in the same manner as the reference realization was built. Scenarios S1, S2, S3, S4, S5, S6 use, as initial realizations, the same 1000 homogenous fields generated based on the bimodal distribution shown in the Figure 3.3.

All scenarios use localization in the application of the NS-EnKF. The distance $a$ in the localization function (Equation 3.9) is set to $40 \mathrm{~m}$ implying that correlation will be zero at a distance of $80 \mathrm{~m}$. This value is chosen after analyzing the experimental cross-covariances of the first batch of realizations. Figure 3.4 shows the localization function. Scenarios S0, S1, S3, S5 do not use covariance inflation, whereas scenarios S2, S4, S6 do use it.

The number of observation piezometers goes from 111 down to 24 for the different scenarios as indicated next. Scenarios S1 and S2 have 111 observation piezometers (see Figure 3.5a), scenarios S0, S3 and S4 have 56 observation piezometers (see Figure 3.5b), and scenarios S5 and S6 have 24 observation piezometers (see Figure 3.5c). In addition, two control piezometers, not used for conditioning, are employed to verify the performance of the NS-EnKF in all the scenarios (see Figure 3.5). The control piezometer number 1 is located in the north-western part of the aquifer, and the control piezometer number 2 is towards the center. 


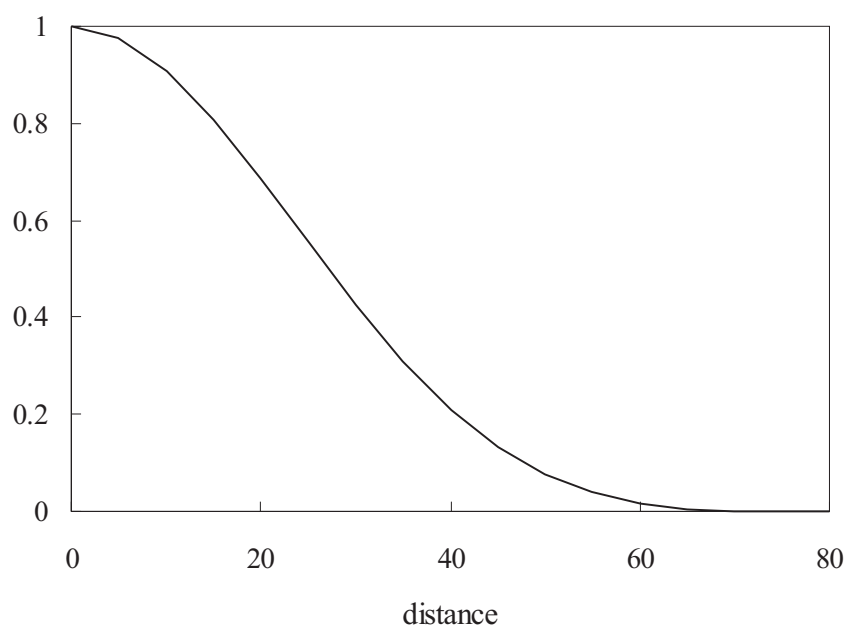

Figure 3.4. The localization function

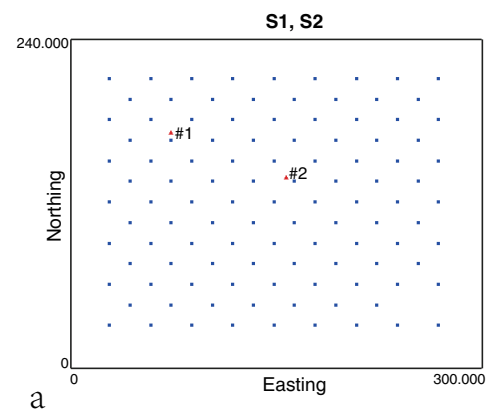

so, S3, s4
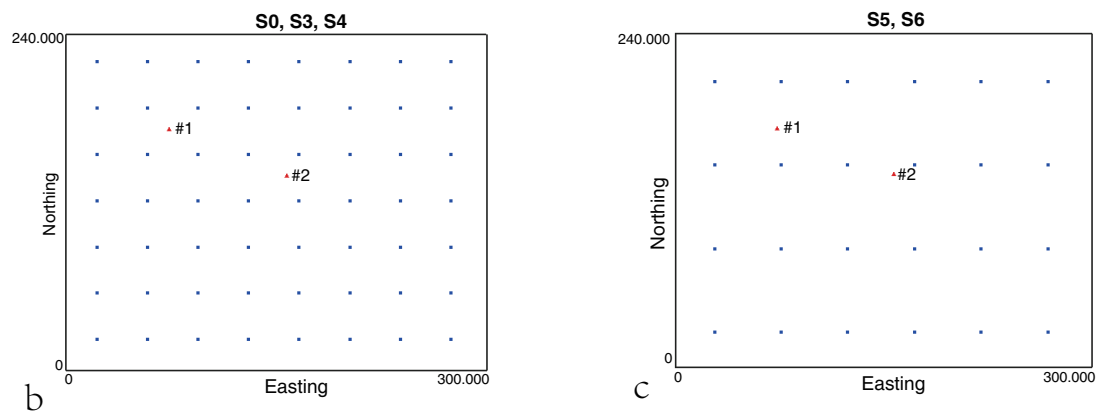

Figure 3.5. Graphs a,b,c show the locations of the 111, 56, 24 observation piezometers, respectively. The blue squares denote observation piezometers, and the red triangles, control piezometers. 


\subsection{Analysis}

We have applied the localized NS-EnKF for the different scenarios described previously assimilating the piezometric observations for the first 60 time steps (67.7 days). We will show the updated log-conductivity fields after the 10th time step (2.4 days) and after the 60th time step. We will also show the piezometric evolution at the control points from time zero until the 100th time step (500 days).

Figure 3.6a displays the log-conductivity histogram for the initial ensemble of heterogeneous realizations used in scenario S0. Figure 3.6b displays the log-conductivity histogram of the updated ensemble of realizations in scenario S0 after the 60th assimilation step. Figure 3.7 displays at the top the log-conductivity histogram for the initial ensemble of homogeneous realizations used in scenarios S1-S6. The corresponding histograms for each scenario after the 60th assimilation step are shown in Figure 3.7a-3.7f. Comparing the updated histograms with the reference one, we can observe that the bimodality is preserved in all scenarios, although only scenarios S0, S2, $\mathrm{S} 4$, and $\mathrm{S} 6$ are able to approximately keep the original proportions between sand and shale.
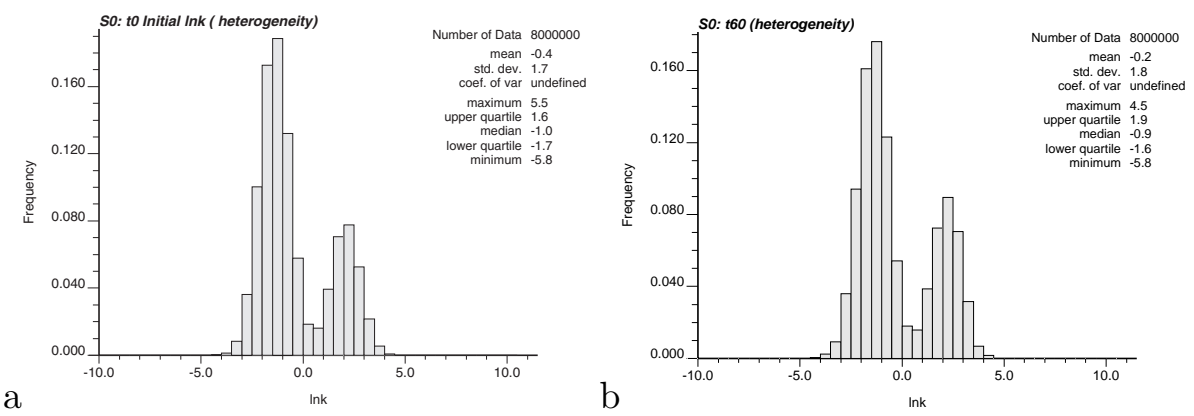

Figure 3.6. Scenario S0. Log-conductivity histograms for the initial ensemble of realizations and for the updated ensemble of realizations after the 60th assimilation step.

Figure 3.8 shows the ensemble mean of the initial log-conductivity fields, together with the ensemble mean of the updated log-conductivity fields after the 10th and 60th assimilation time step for scenario S0. Similarly Figure 3.9 shows the ensemble variance for the same sets of log-conductivity in Figure 3.8.

The ensemble mean and the ensemble variance of the initial log-conductivities for scenarios S1-S6 are not shown, since they are the same as Figure 3.8a and Figure 3.9a, respectively. Figures 3.11 and 3.12 show the ensemble means of the updated fields after the 10th and 60th time step, respectively. Similarly, Figures 3.13 and 3.14 show the corresponding ensemble variances. 


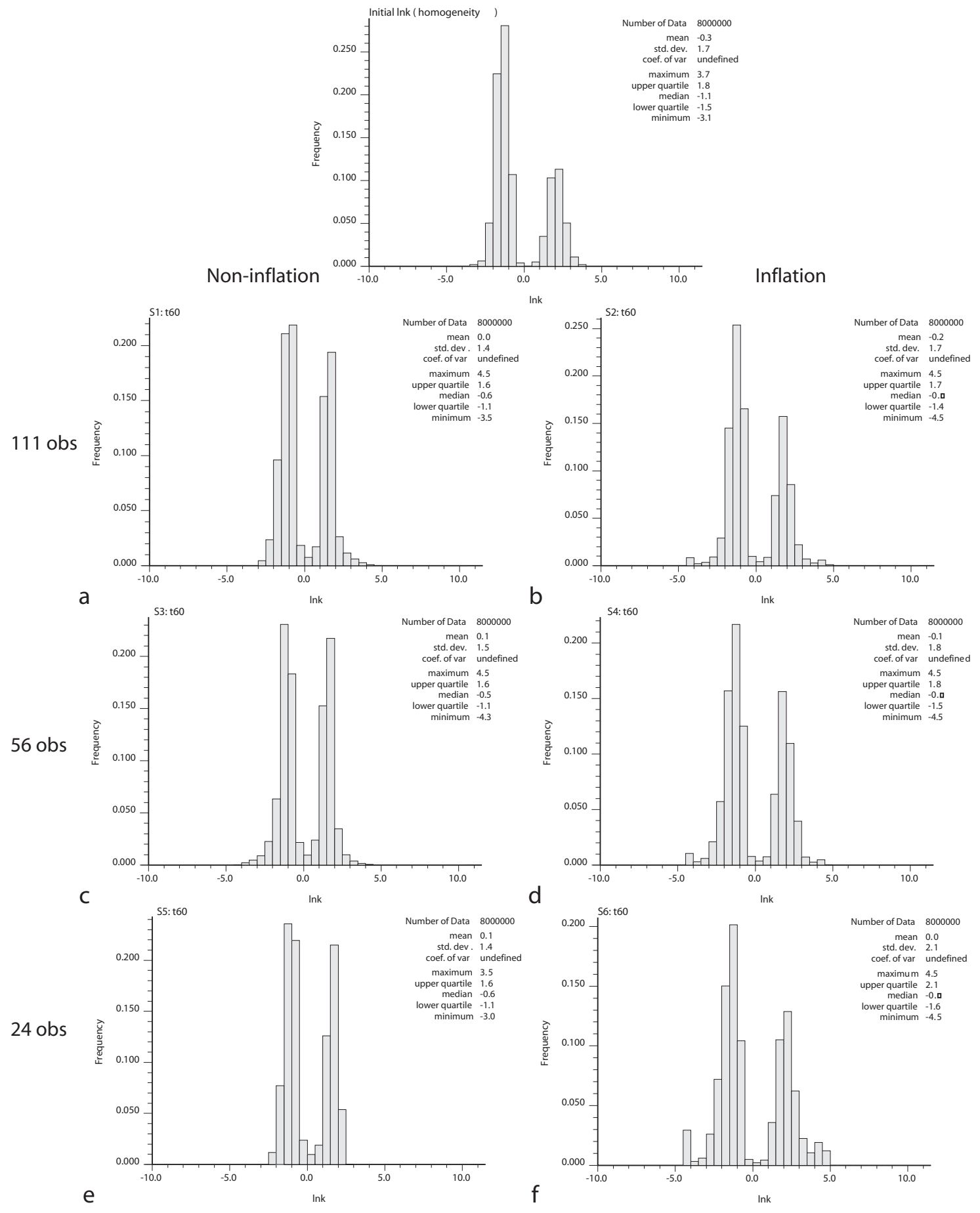

Figure 3.7. Scenarios S1-S6. Log-conductivity histograms. The top one shows the histogram for the initial ensemble of homogeneous realizations used in scenarios S1-S6; graphs a to f show the histograms for the corresponding scenarios after the 60th data assimilation step. The scenarios without covariance inflation are shown in the left column; the scenarios with covariance inflation are shown in the right column. The number of observation piezometers used is indicated in the left. 


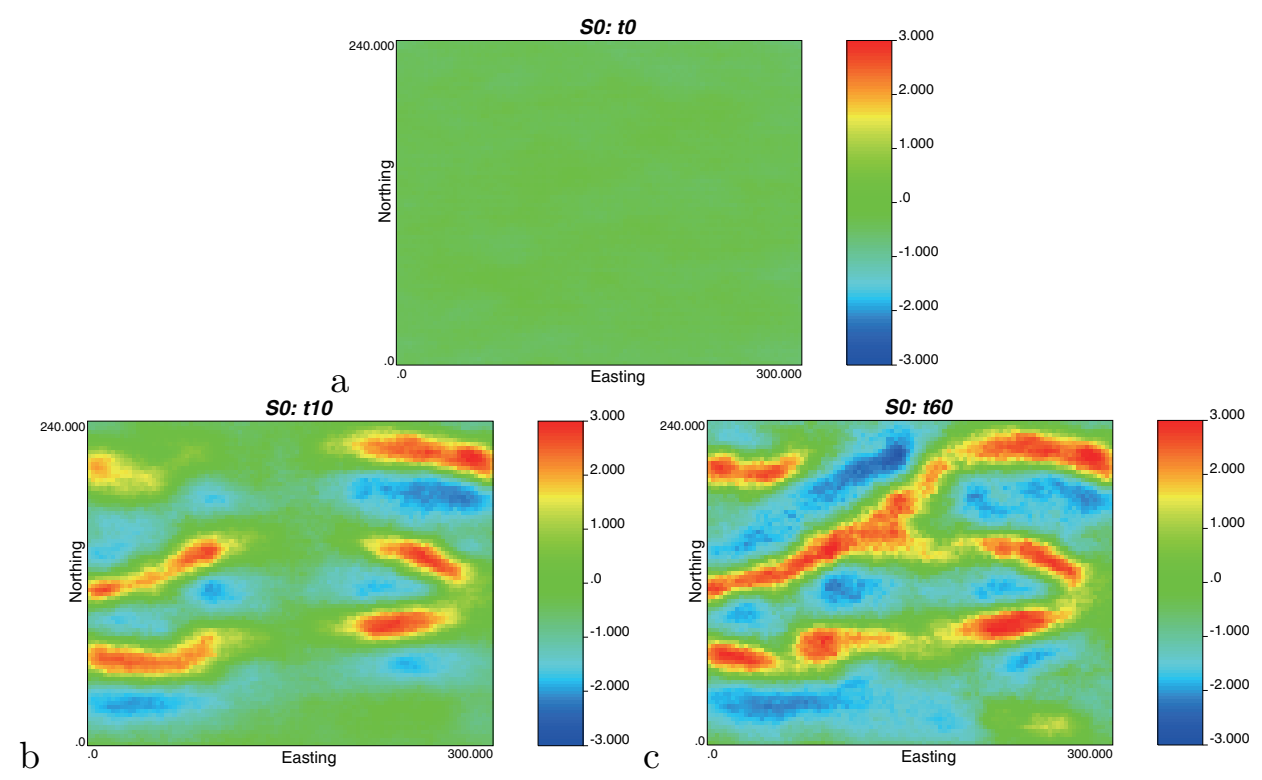

Figure 3.8. Scenario S0. Ensemble mean of $\ln K$ for the initial realizations and after assimilating heads at the 10th and 60th time steps.

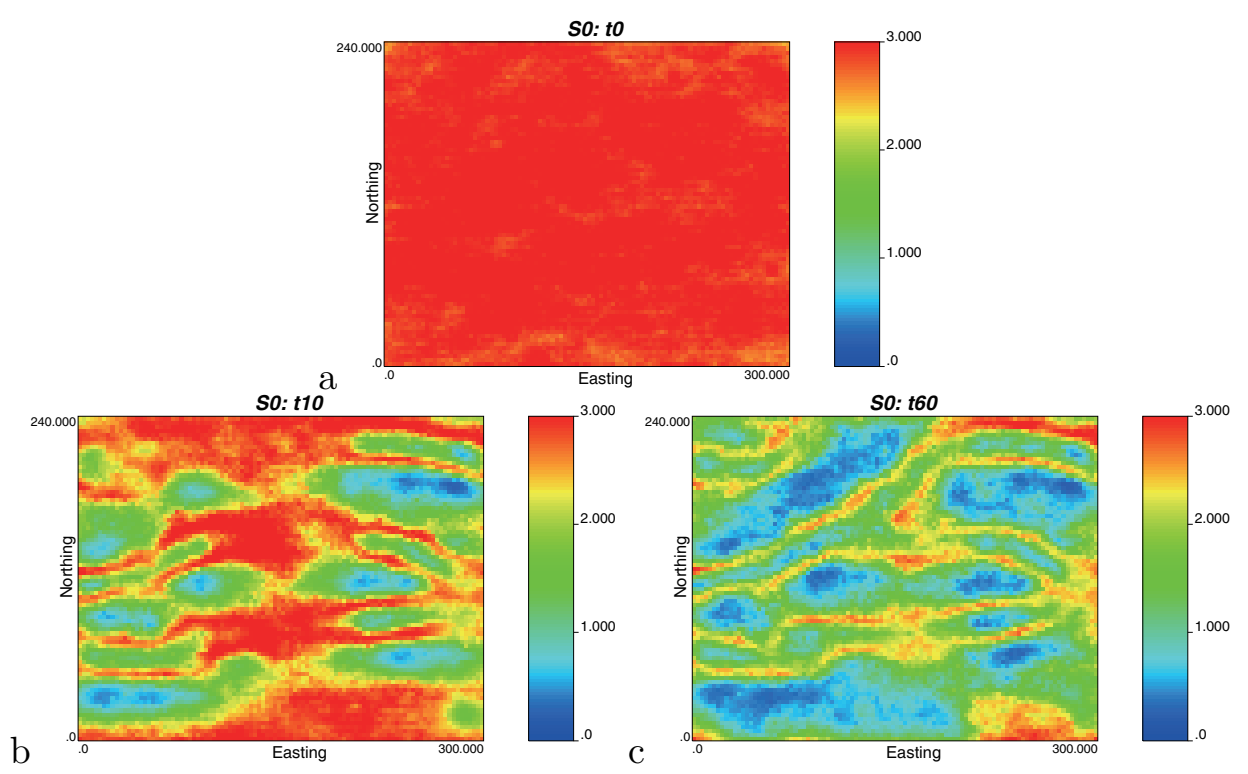

Figure 3.9. Scenario S0. Ensemble $\ln K$ variance for the initial realizations and after assimilating heads at the 10th and 60th time steps. 
The initial ensemble means in Figure 3.8a and Figure 3.10a are homogeneous with a value equal to the prior mean (even for scenario S0), since the initial realizations are unconditional. For the same reason, the initial ensemble variances in Figure 3.9a and Figure 3.10b are also homogeneous with a value equal to the prior variance.
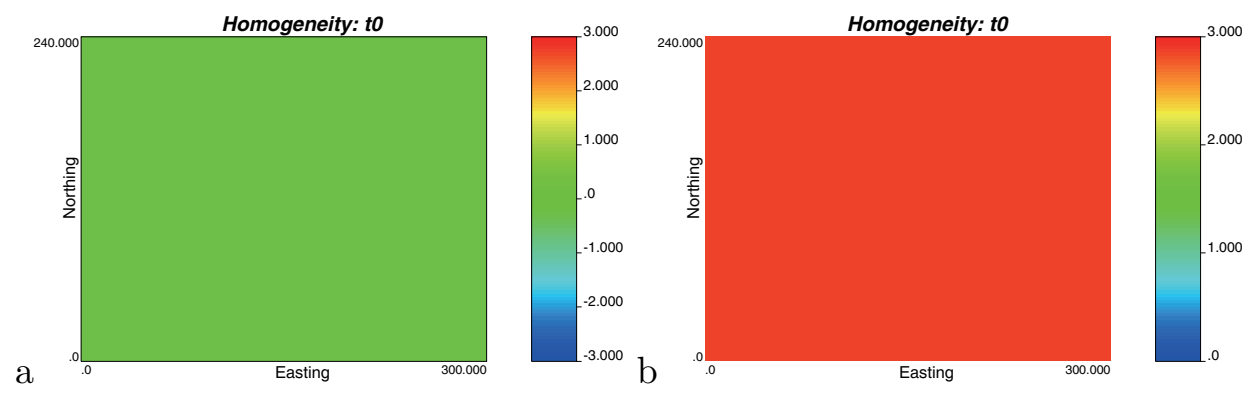

Figure 3.10. Maps a,b show the ensemble mean and ensemble variance of the initial realizations for the scenarios with initial homogenous fields (S1-S6).

Figure 3.8 and Figure 3.9 replicate the results by Zhou et al. (2011) who introduced the NS-EnKF algorithm. We can see how, as time progresses, the main channel features in the reference field are better delineated in the ensemble mean maps, and the ensemble variance decreases. Since the fastest piezometric head changes are close to the east and west boundaries, the channel features close to these boundaries can be already identified at the 10th time step. It was precisely the evolution of the ensemble mean map as a function of time seen in the these figures, what disclosed to us the importance of the transient piezometric head for hydraulic conductivity characterization. For this reason, this paper focuses in the power of assimilating transient piezometric heads using the NS-EnKF algorithm for the case in which we do not have any information about the spatial variability of hydraulic conductivities.

Figures 3.11a,c,e, and 3.12a,c,e show the ensemble means for the scenarios in which no covariance inflation has been implemented. Correspondingly, Figures 3.13a,c,e, and 3.14a,c,e show the ensemble variances for these scenarios. We notice that the implementation of the localized NS-EnKF with homogeneous initial fields results in filter inbreeding very quickly. This can be identified in the variance maps in Figures 3.14a,c,e, which are almost zero everywhere. Even though, after the 60th time step, some of the channel features can be identified when using 111 piezometers, we discarded these results as acceptable due to filter inbreeding. And, for this reason, we implemented covariance inflation into the localized NS-EnKF.

Figures $3.11 \mathrm{~b}, \mathrm{~d}, \mathrm{f}$, and $3.12 \mathrm{~b}$,d,f show the ensemble means for the scenarios in which covariance inflation has been implemented. Correspondingly, Figures $3.13 \mathrm{~b}, \mathrm{~d}$,f, and $3.14 \mathrm{~b}, \mathrm{~d}$,f show the ensemble variances in these sce- 


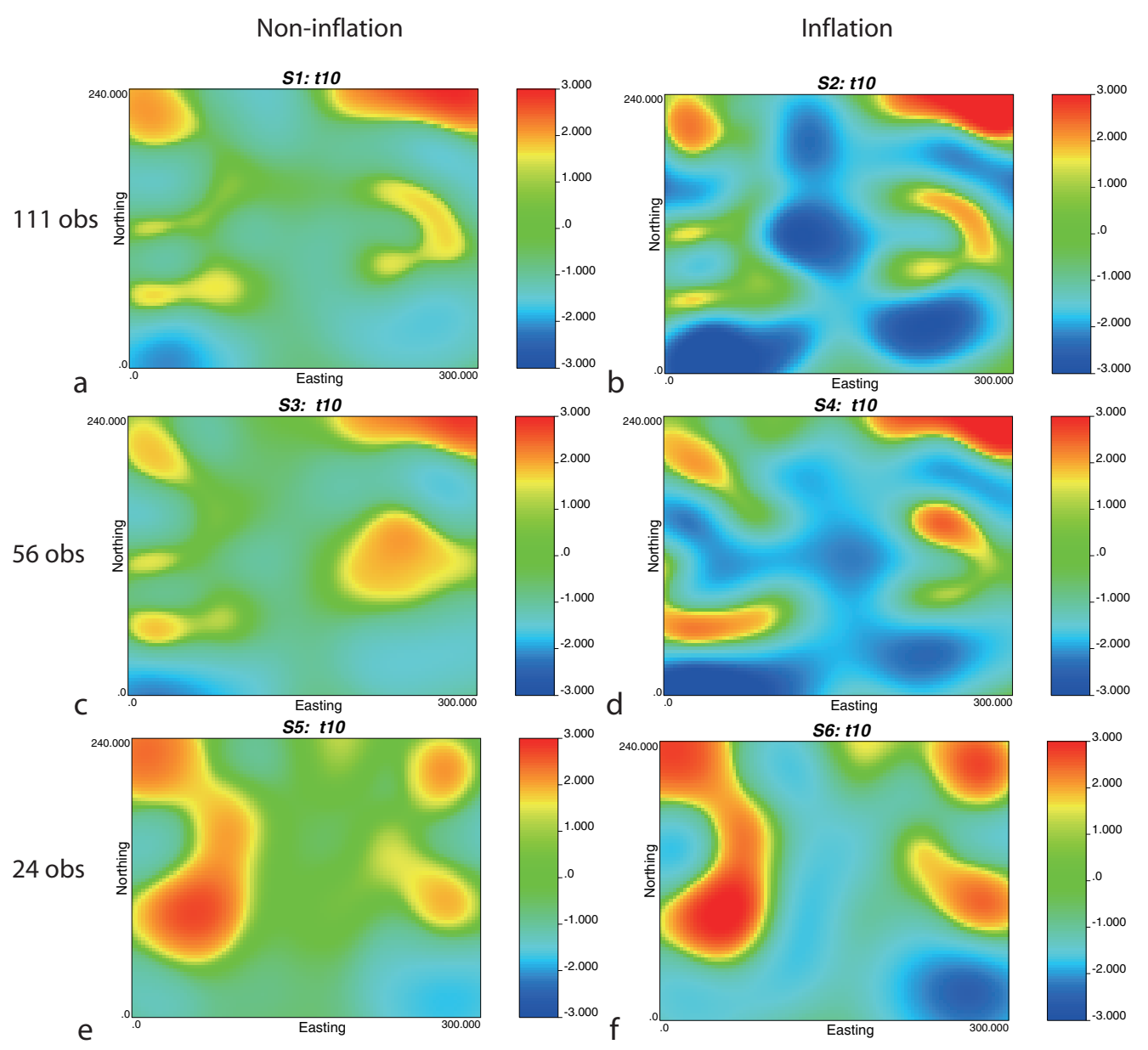

Figure 3.11. Scenarios S1-S6. Log-conductivity ensemble mean computed after the 10th time step. 


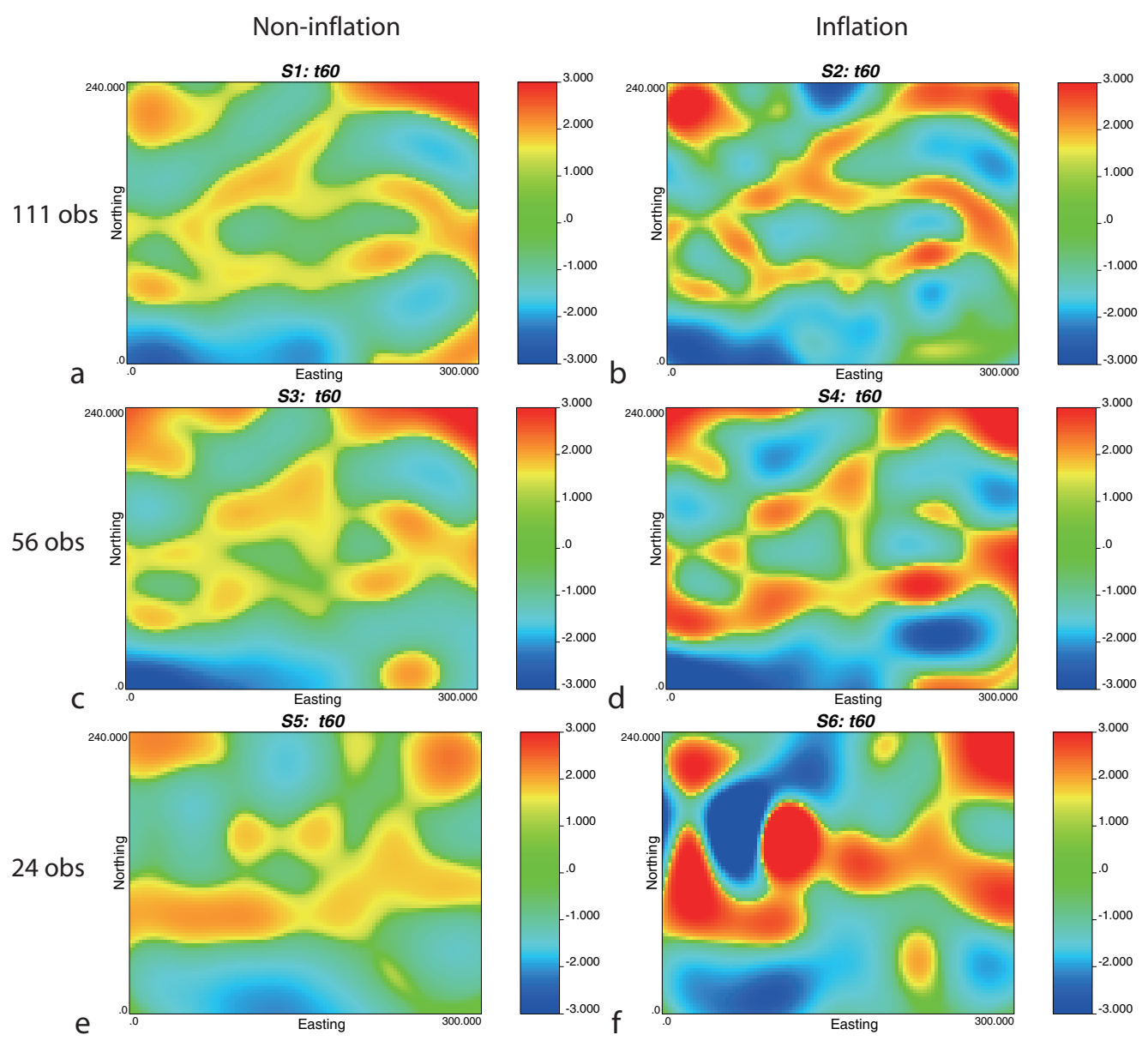

Figure 3.12. Scenarios S1-S6. Log-conductivity ensemble mean computed after the 60 th time step. 

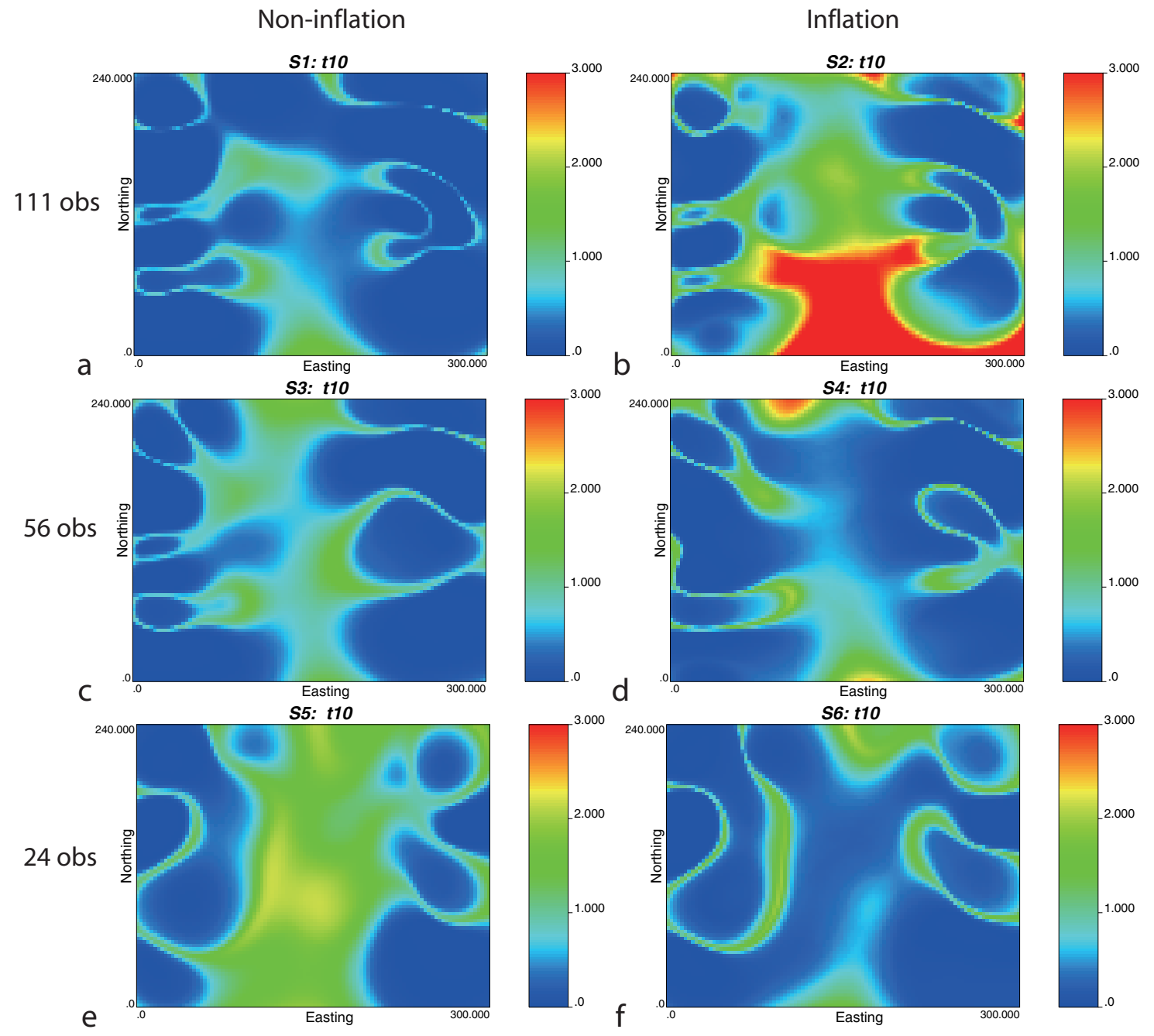

Figure 3.13. Scenarios S1-S6. Log-conductivity ensemble variance computed after the 10th time step. 


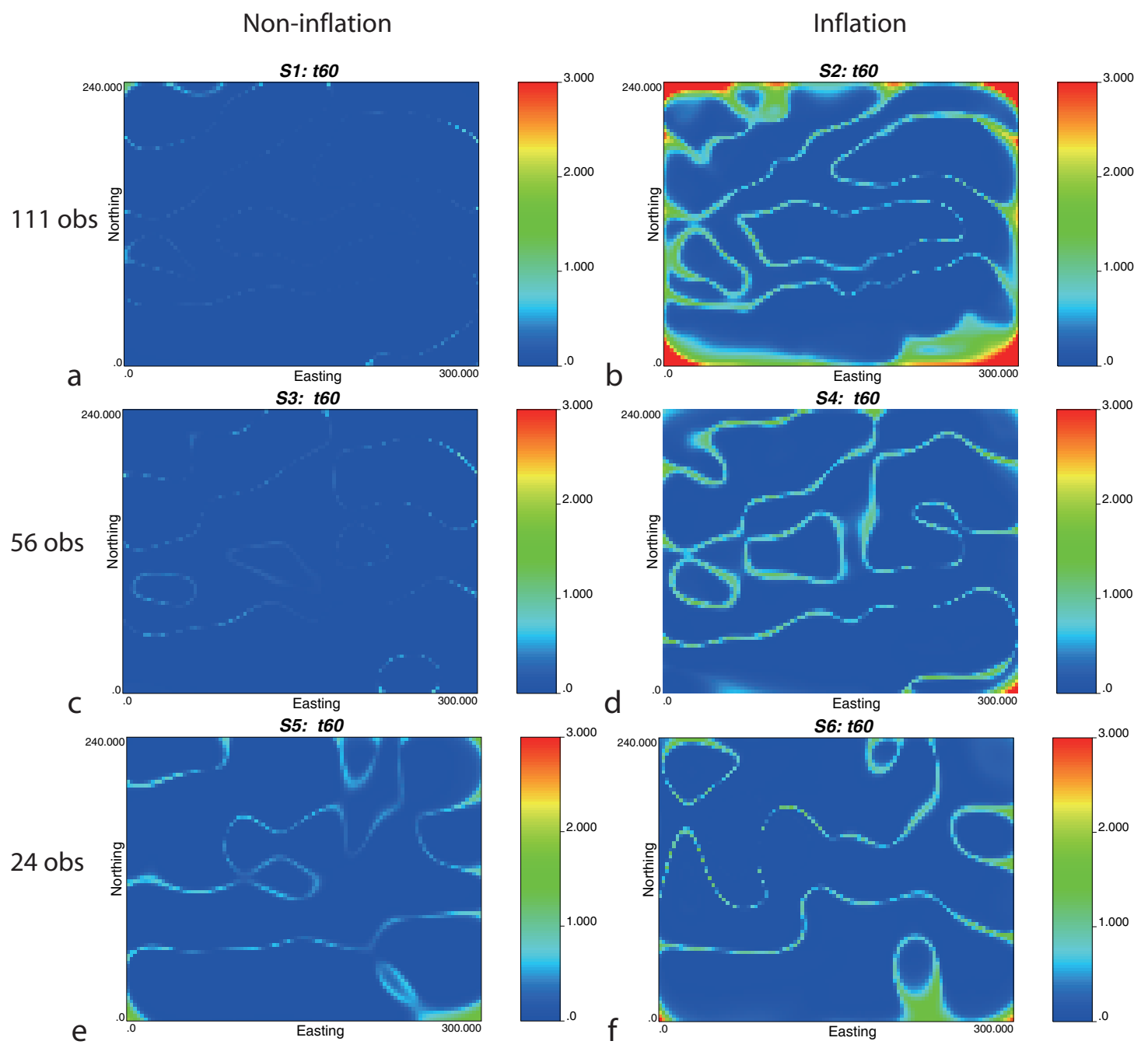

Figure 3.14. Scenarios S1-S6. Log-conductivity ensemble variance computed after the 60 th time step. 
narios. When using 111 piezometers and covariance inflation after 60 time steps, the ensemble mean captures very well the main features of the reference field (see Figure 3.12b). If the number of piezometers is reduced to 56 , the method can still capture the general position of the channels, but with less accuracy than in the previous case (see Figure 3.12d). However, if we reduce the number of piezometers down to 24, then the characterization of the channels is very poor. As in scenario S0 after 10 time steps, in scenarios S1-S6, we can start to see the appearance of the channels in the ensemble means of the updated fields. For these scenarios, in which covariance inflation was implemented, the ensemble variance after 60 time steps is too small indicating some filter inbreeding.

The issue of filter inbreeding is better analyzed by looking at the ratio of the root mean square error $(R M S E)$ to the ensemble spread $(E S)$, where $R M S E$ and $E S$ are defined as follows:

$$
R M S E=\sqrt{\frac{1}{n} \sum_{i=1}^{n}\left(s_{i}^{r e f}-\left\langle s^{a}\right\rangle_{i}\right)^{2}},
$$

where $n$ is the number of model elements; $s_{i}^{r e f}$ is the value of the reference field at node $i ;\left\langle s^{a}\right\rangle_{i}$ is the ensemble mean of the updated fields, and

$$
E S=\sqrt{\frac{1}{n} \sum_{i=1}^{n} \sigma_{i}^{2}},
$$

where $\sigma_{i}$ is the ensemble variance of the updated fields at node $i$.

The $R M S E$ measures how well the ensemble average map reproduces the reference one, the smaller the $R M S E$, the better the reproduction. Yet, we know that the ensemble average map can only be a smooth representation of the spatial heterogeneity, and consequently it can never be zero. The ES measures the degree of variability across the different realizations. When $E S$ is too close to zero, it indicates that the realizations have collapsed and filter inbreeding occurs. Liang et al. (2011) show that a good way to check the degree of filter inbreeding is by analyzing the ratio of $R M S E$ to $E S$, which, in ideal conditions, should be 1 .

Figure 3.15 shows the evolution of the RMSE for all scenarios computed on the updated log-conductivity fields after each assimilation step. We can see how, except for S6, the $R M S E$ decreases with time. The smallest values are found for scenario S0, followed by scenario S2. Figure 3.16 shows the evolution with time of the ratio of $R M S E$ to $E S$. In this figure, we can clearly see how for scenario S0 this ratio converges quickly to 1, indicating that there is no filter inbreeding. On the other hand, filter inbreeding is very high for scenarios S1, S3, S5 (the ones without covariance inflation), and it is less pronounced for scenarios $\mathrm{S} 2, \mathrm{~S} 4, \mathrm{~S} 6$ (the ones with covariance 
inflation). As already noticed in Figure $3.12 \mathrm{~b}$, scenario S2 provides the best results.

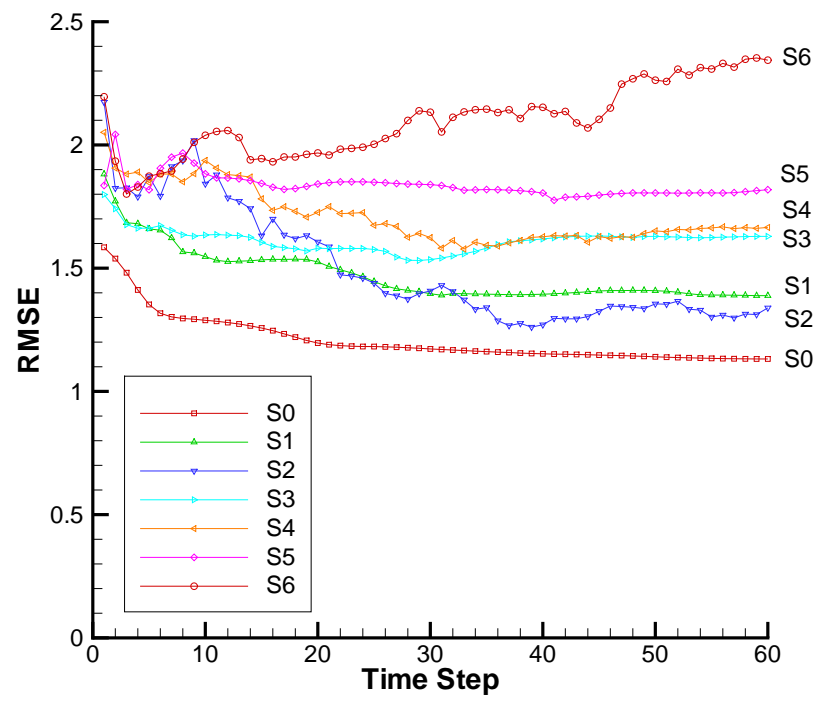

Figure 3.15. $R M S E$

Next, we analyze the reproduction of the piezometric heads at the control piezometers. Figure 3.17 shows the evolution of the piezometric heads at control piezometers 1 and 2 for the initial log-conductivity fields; in the top row, the evolution in the heterogeneous fields used in scenario S0, and in the bottom row, the evolution in the homogeneous fields used in the other scenarios. The figure also shows the evolution of heads in the reference field and the average of the individual realizations. Figure 3.18 shows the evolution at the two control points in the updated fields after 60 time steps for scenario S0. Figure 3.19 and Figure 3.20 shows the evolution of heads at control piezometers 1 and 2, respectively, in the updated fields after 60 time steps for scenarios S1-S6. Notice that for Figures 3.18, 3.19 and 3.20 the assimilation period lasts only until day 67.7 , beyond that the logconductivity fields are not updated anymore.

We can see in Figure 3.17 that with no conditioning to conductivity and without assimilating any piezometric head the spread of the responses of piezometric head is extremely large. The localized NS-EnKF with initial heterogeneous fields (scenario S0) does a good job in reducing the spread of the piezometric head curves with the conductivity fields updated up to the 60th assimilation time step. However, there is still a small bias between the reference values and the ensemble mean results. 


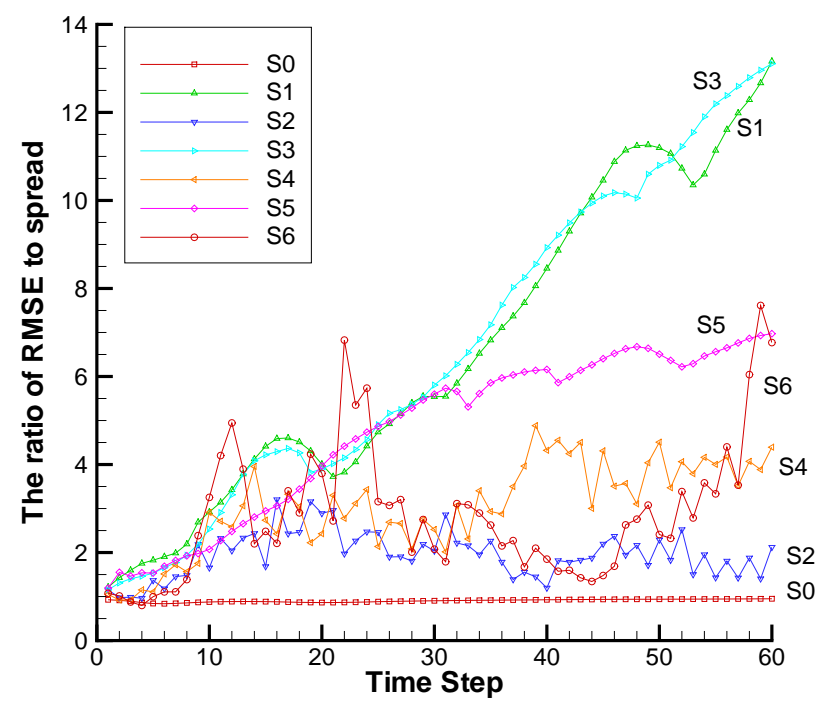

Figure 3.16. The ratio of $R M S E$ to $S E$

The evolution of the piezometric heads computed on the updated fields after the 60th assimilation time step in both control piezometers is very similar for all scenarios. The spread is reduced very much with respect to the spread in the initial fields, although for some scenarios like S1, or S3, the reduction is too large due to the filter inbreeding. Scenario S2, which performed best for log-conductivity reproduction, is the one displaying the largest spread among the different realizations but also the largest bias between the reference values and the ensemble average.

In order to analyze the characterization of the log-conductivity fields in the different scenarios, we are going to perform two additional checks, one involving the advective transport of an inert solute, and the other one based on the analysis of some connectivity functions. For these checks, we will use the updated log-conductivity fields after the 60th assimilation time step.

For the transport exercise, we release 10,000 particles along an injection line at $x=10 \mathrm{~m}$ and we track them to the two control planes at $x=100$ $\mathrm{m}$ and $x=280 \mathrm{~m}$ using the random walk particle tracking program RW3D (Fernàndez-Garcia et al., 2005) (see Figure 3.2). Porosity is assumed constant and equal to 0.3. Figure 3.21 shows the breakthrough curves (BTCs) corresponding to scenario S0. Figure 3.22 shows the BTCs at the first control plane for scenarios S1-S6, and Figure 3.23 shows the BTCs at the second control plane. 


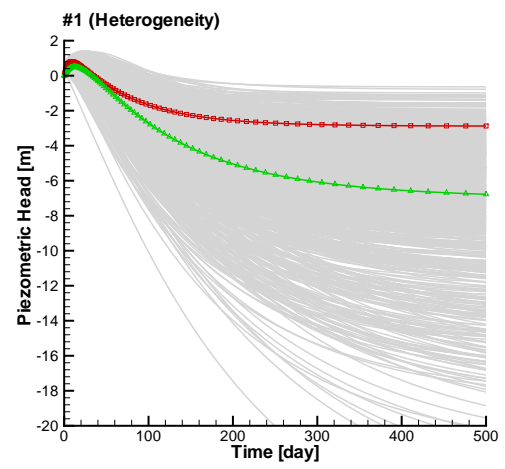

a

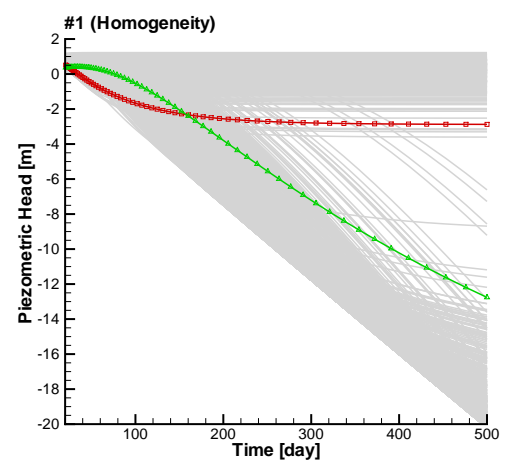

$\mathrm{b}$
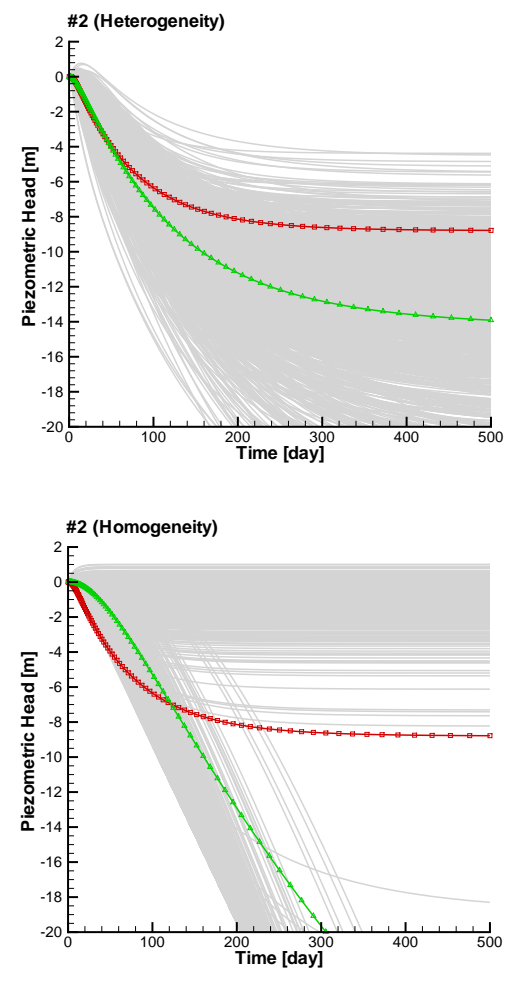

Figure 3.17. Graphs a,b show the piezometric head time evolution of the initial ensemble of heterogenous log-conductivity realizations at the control piezometers 1 and 2, respectively; graphs c,d show the piezometric head time evolution on the initial homogenous realizations at the two control piezometers. The red square line corresponds to the piezometric head time evolution in the reference, the green triangle line corresponds to the mean of the ensemble, and the gray lines correspond to the realizations. 
a

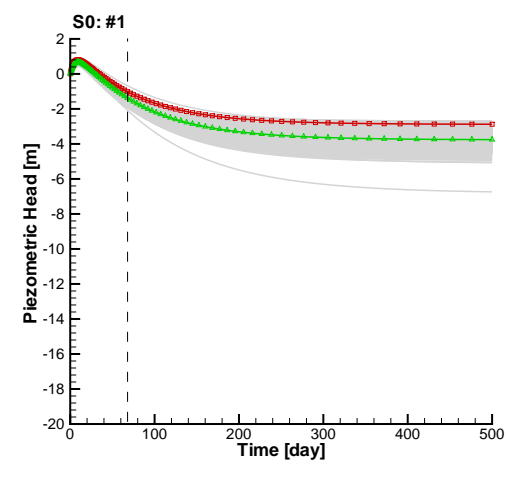

$\mathrm{b}$

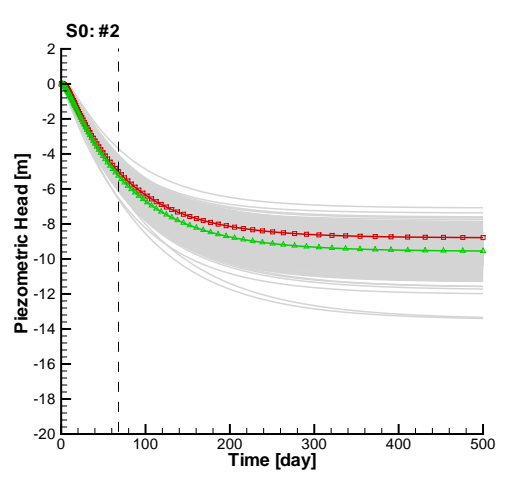

Figure 3.18. Scenario S0. The piezometric head time evolution after the 60th time step for the two control piezometers. The red square line corresponds to the piezometric head time evolution in the reference, the green delta line corresponds to the mean of the ensemble, the gray lines correspond to the realizations and the vertical dashed lines indicate the conditioning period.

Again, scenario S0 is the one that performs best since the reference BTCs are within the $90 \%$ confidence interval for both control planes, and the median BTCs do an acceptable job in reproducing the reference BTCs. The non inflation scenarios display an extremely narrow $90 \%$ confidence interval, although they are able to reproduce the reference BTCs for control plane 2. Of the inflation scenarios both $\mathrm{S} 2$ and $\mathrm{S} 4$ give good results both in terms of the confidence intervals and the approximation of the reference BTC by the median. The behavior of scenario S6 is odd, particularly when compared with S5, since S5 is able to reproduce moderately well the BTCs for both control planes (with a very narrow band of uncertainty) and S6 fails completely, displaying a transport behavior much slower than in all other scenarios. This behavior must be due to the covariance inflation and the low number of conditioning points, such an inflation may result in an overall higher variability that masks the presence of the conductivity channels.

All in all, transport results could be very much dependent on the reference field used for the analysis, and should be interpreted in this view.

For the connectivity exercise, we are going to analyze the connectivity of high conductivity values in the horizontal direction. Of the different methods proposed to evaluate connectivity (e.g., Western et al., 2001; Knudby and Carrera, 2005; Neuweiler and Cirpka, 2005), we choose the one proposed by Stauffer and Aharony (1994). Before computing the connectivity of each field we need to convert the continuous log-conductivity fields into binary fields using the indicator transform function

$$
I(x)= \begin{cases}1, & \text { if } \ln K \geq 0 \\ 0, & \text { otherwise }\end{cases}
$$




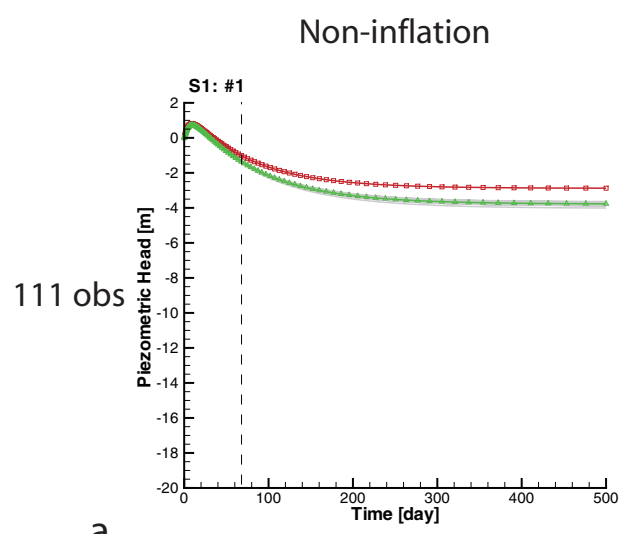

Inflation

a

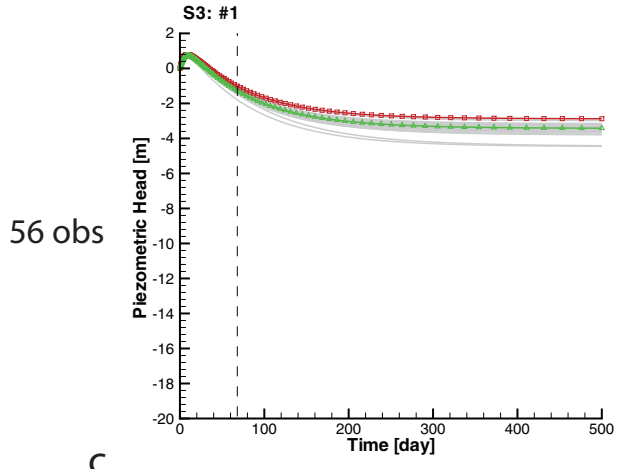

C

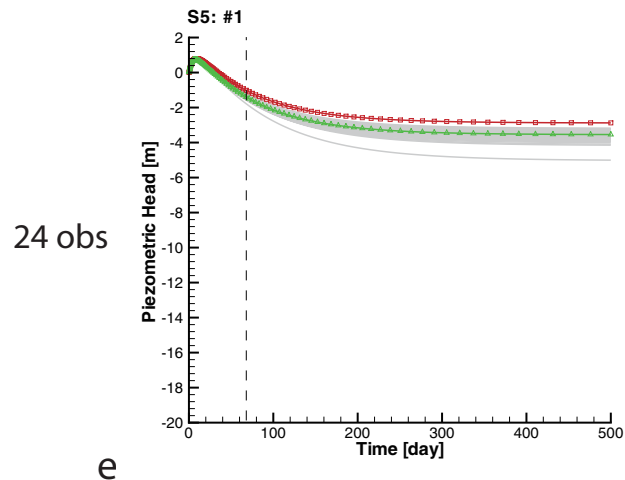

$\mathrm{b}$
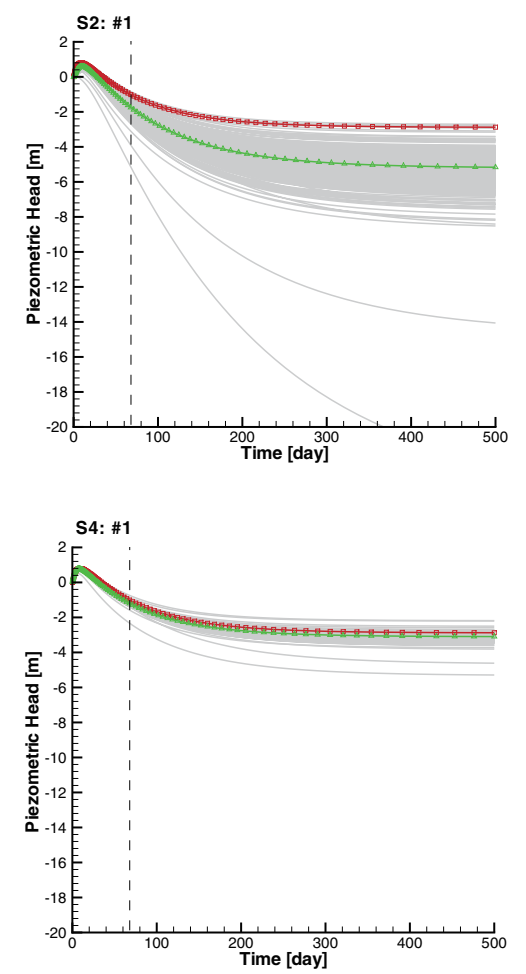

d

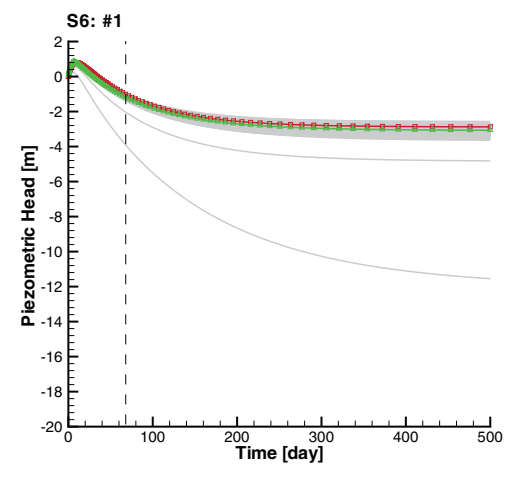

Figure 3.19. Scenarios S1-S6. The piezometric head time evolution at the control piezometer 1 after the 60th time step. The red square line corresponds to the piezometric head time evolution in the reference, the green delta line corresponds to the mean of the ensemble, the gray lines correspond to the realizations and the vertical dashed lines indicate the conditioning period. 

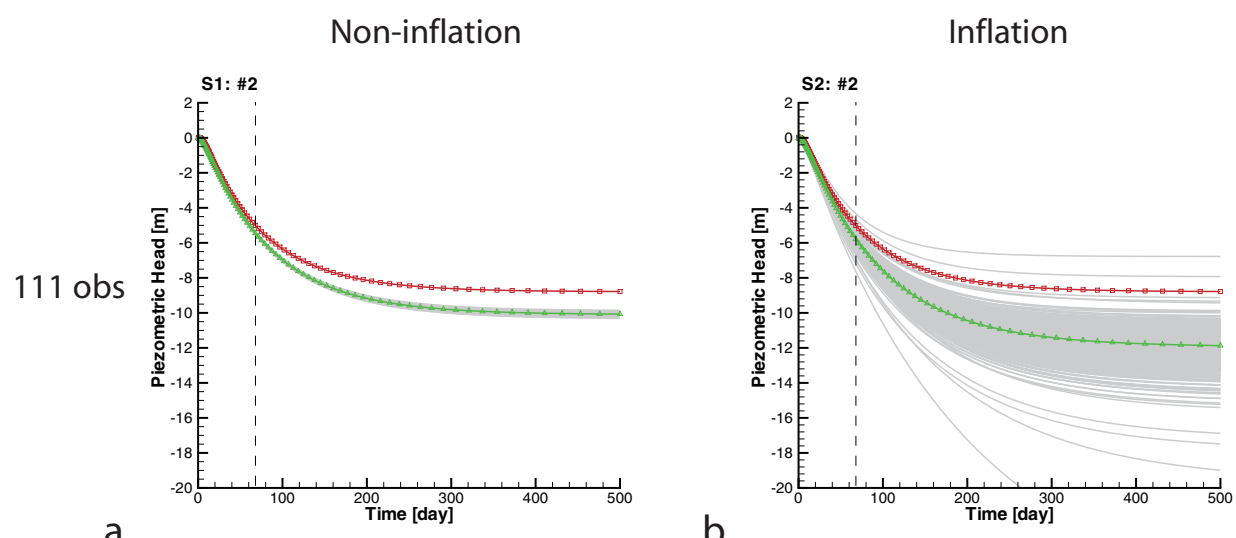

a

b
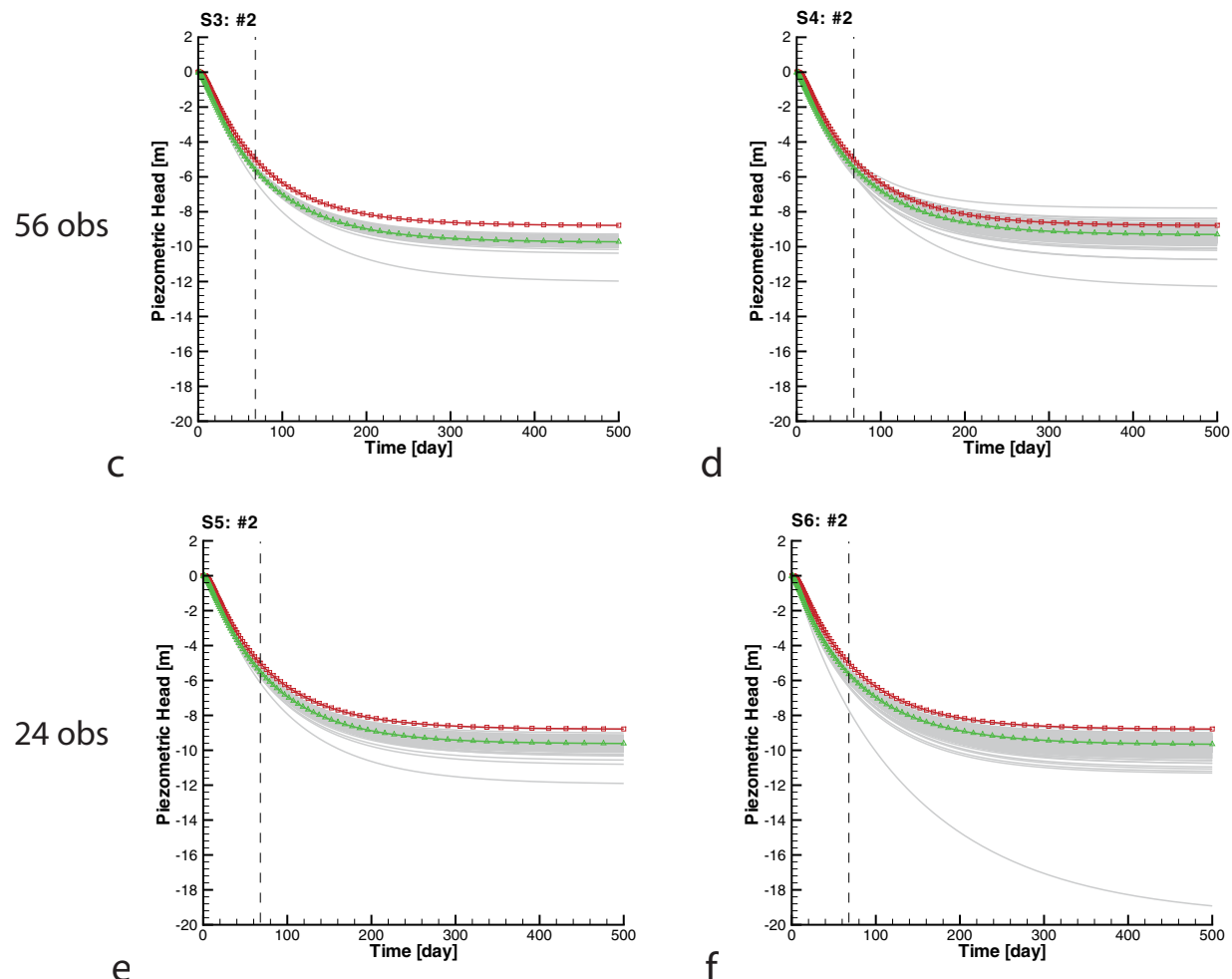

d

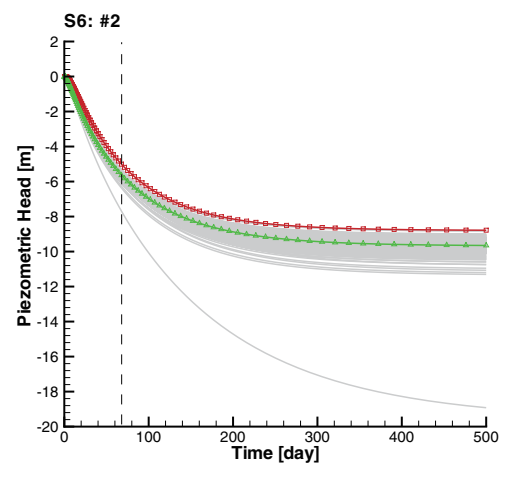

Figure 3.20. Scenarios S1-S6. The piezometric head time evolution at the control piezometer 2 after the 60 th time step. The red square line corresponds to the piezometric head time evolution in the reference, the green delta line corresponds to the mean of the ensemble, the gray lines correspond to the realizations and the vertical dashed lines indicate the conditioning period. 

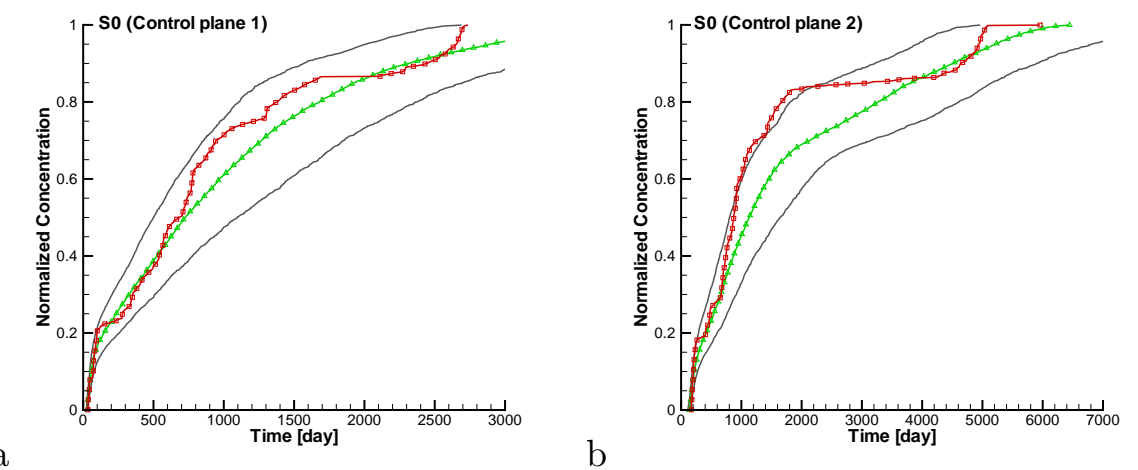

Figure 3.21. Graphs a,b show the BTCs at the two control planes for scenario S0. The red square line corresponds to BTCs in the reference. The black lines correspond to the 5 and 95 percentiles of all realization BTCs, and the green delta line corresponds to the median.

where we chose the threshold value $\ln K=0$ because it separates sand from shale in the reference histogram (Figure 3.3). The program CONNEC3D (Pardo-Igúzquiza and Dowd, 2003) computes the connectivity following the method by Stauffer and Aharony (1994) as the probability that two points with log-conductivities larger than zero horizontally separated by a certain distance are connected by a continuous path of log-conductivities larger than zero. Figures 3.24 and 3.25 show the connectivity curves for the high conductivities as a function of their horizontal separation distance. Both figures show the connectivity curves computed in all realizations together with the connectivity curve computed in the reference field, and the mean of the curves. Figure 3.24 shows the connectivity curves for the initial heterogeneous conductivity realizations and for the updated conductivity realizations after the 60th assimilation time step for scenario S0. Figure 3.25 shows the connectivity functions for the updated conductivity realizations after the 60th assimilation time step for scenarios S1-S6. The connectivity functions for the initial homogeneous fields are not displayed since the connectivity in a homogeneous field is always perfect.

Analyzing Figures 3.24 and 3.25 we can arrive at the same conclusions as before. The spread of the curves for the non inflation scenarios is too small. The fact that the connectivity functions for scenarios S1 and S3 are so close to the reference connectivity function may be the explanation why the BTCs are also so well reproduced for these scenarios. Scenarios S2 and S4 show a larger spread than the non inflation scenarios, yet, the envelope of individual functions encloses the reference function, and its mean is an acceptable approximation of the reference. 
Non-inflation

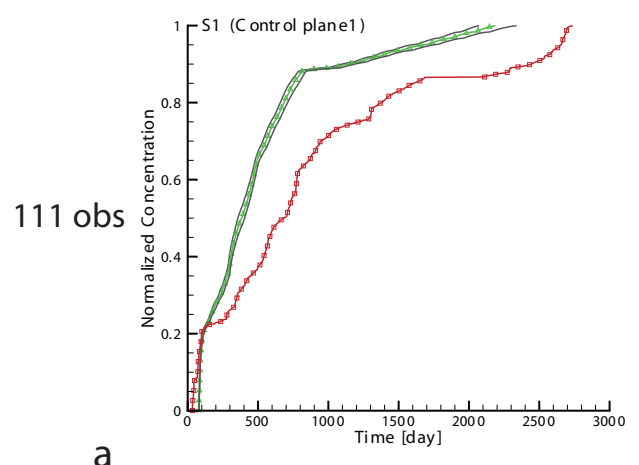

a

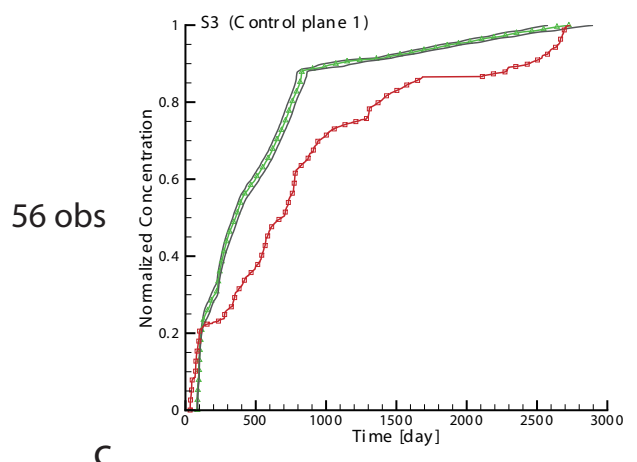

C

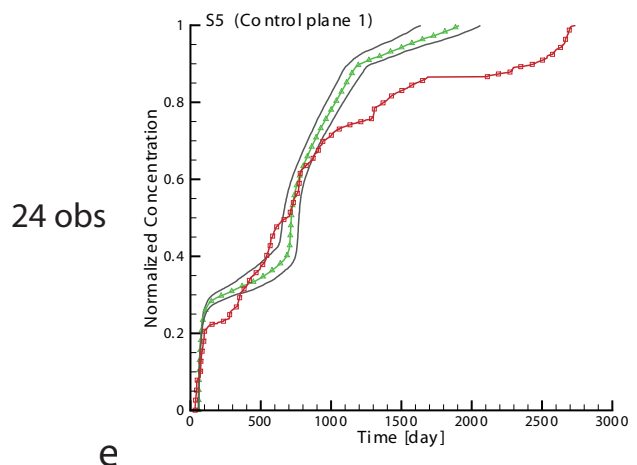

b
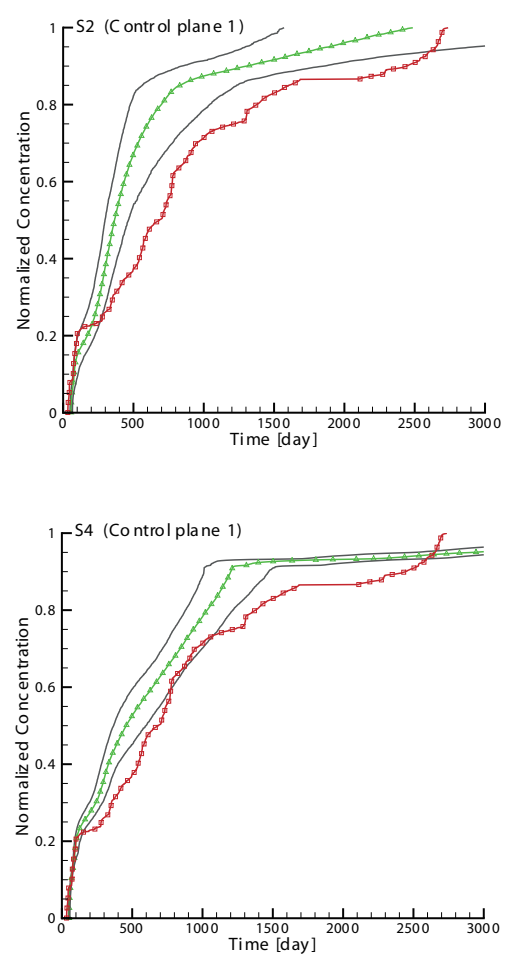

d

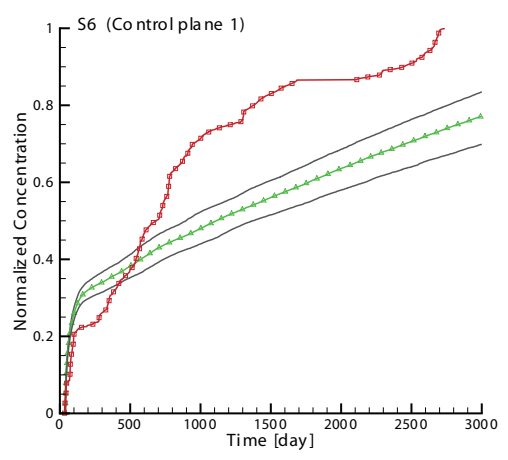

Figure 3.22. Scenarios S1-S6. The BTCs at the first control plane. The red square line corresponds to BTCs in the reference. The black lines correspond to the 5 and 95 percentiles of all realization BTCs, and the green delta line corresponds to the median. 

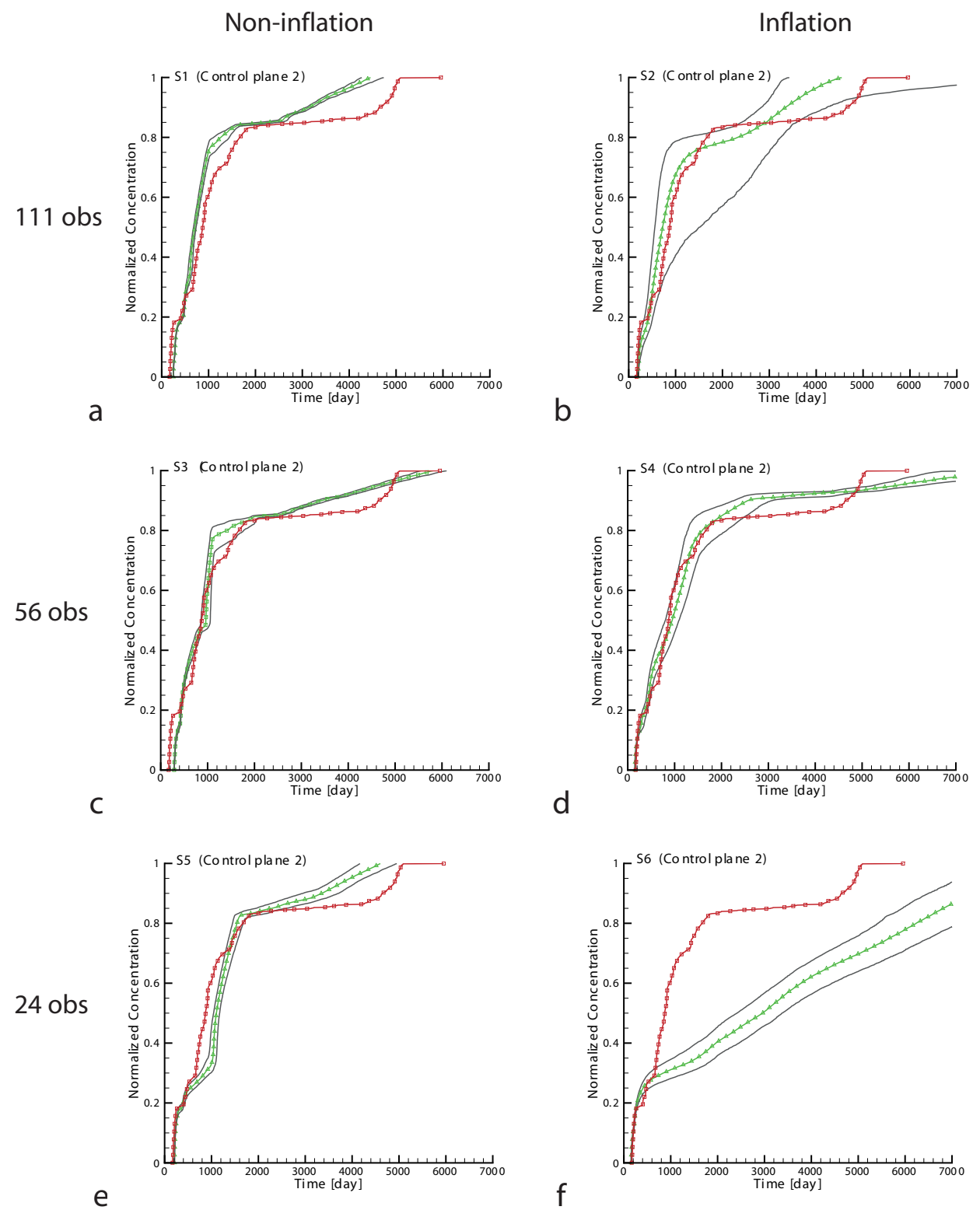

Figure 3.23. Scenarios S1-S6. The BTCs at the second control plane. The red square line corresponds to BTCs in the reference. The black lines correspond to the 5 and 95 percentiles of all realization BTCs, and the green delta line corresponds to the median. 


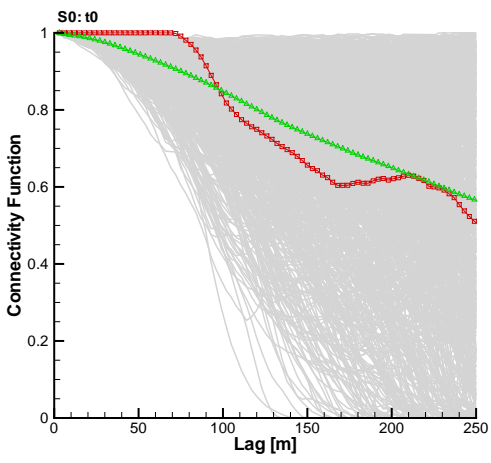

\section{a}

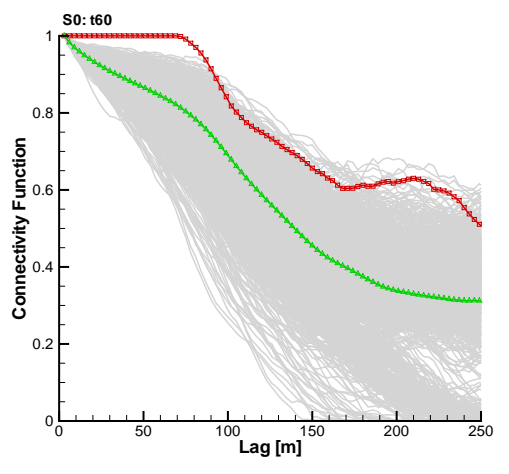

Figure 3.24. Scenarios S0. Connectivity curves for the initial ensemble of realizations and for the updated realizations after the 60th assimilation time step. The red square line corresponds to the connectivity curve in the reference, the green delta line corresponds to the mean of the ensemble, and the gray lines correspond to the individual realizations. 

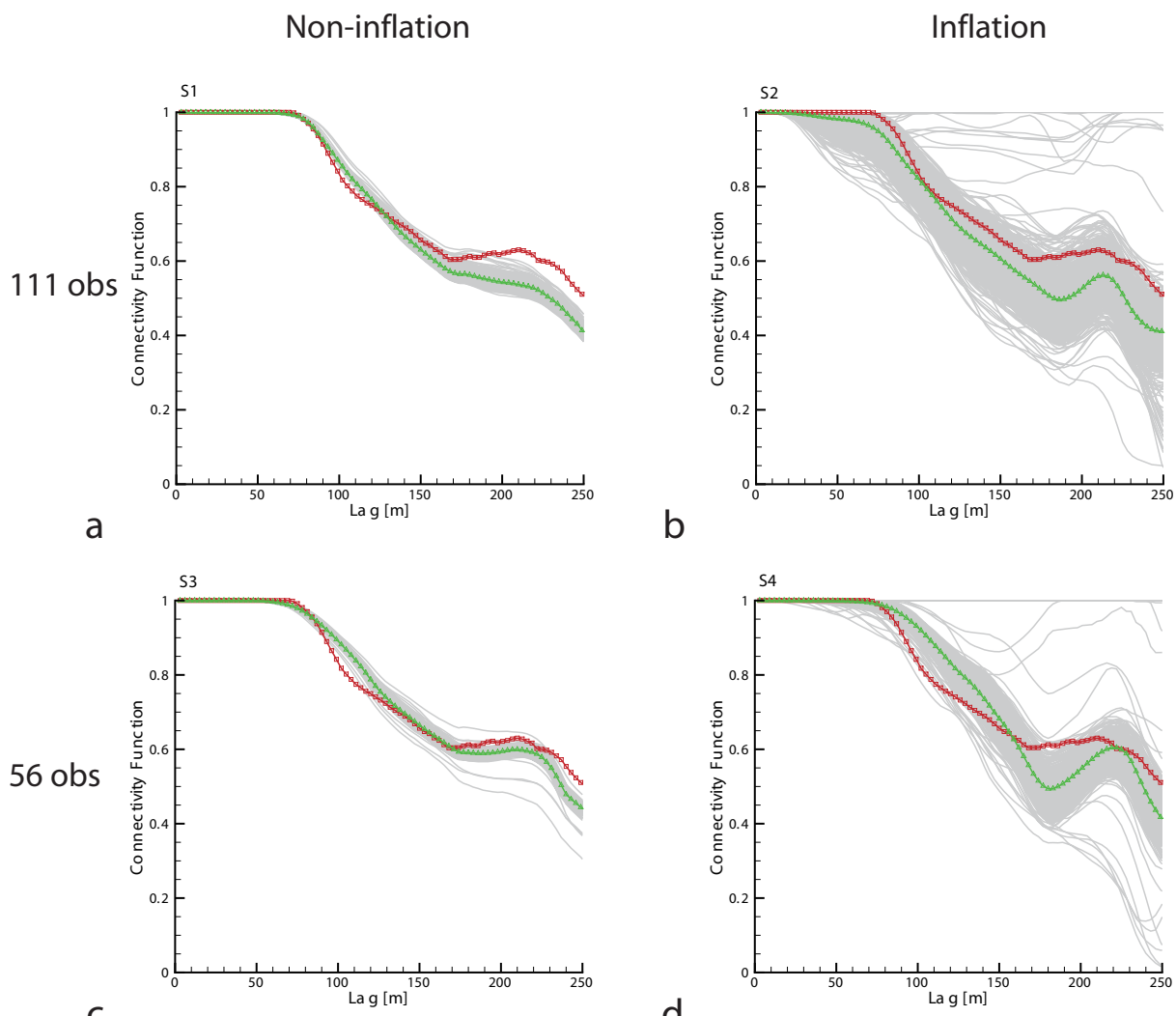

b
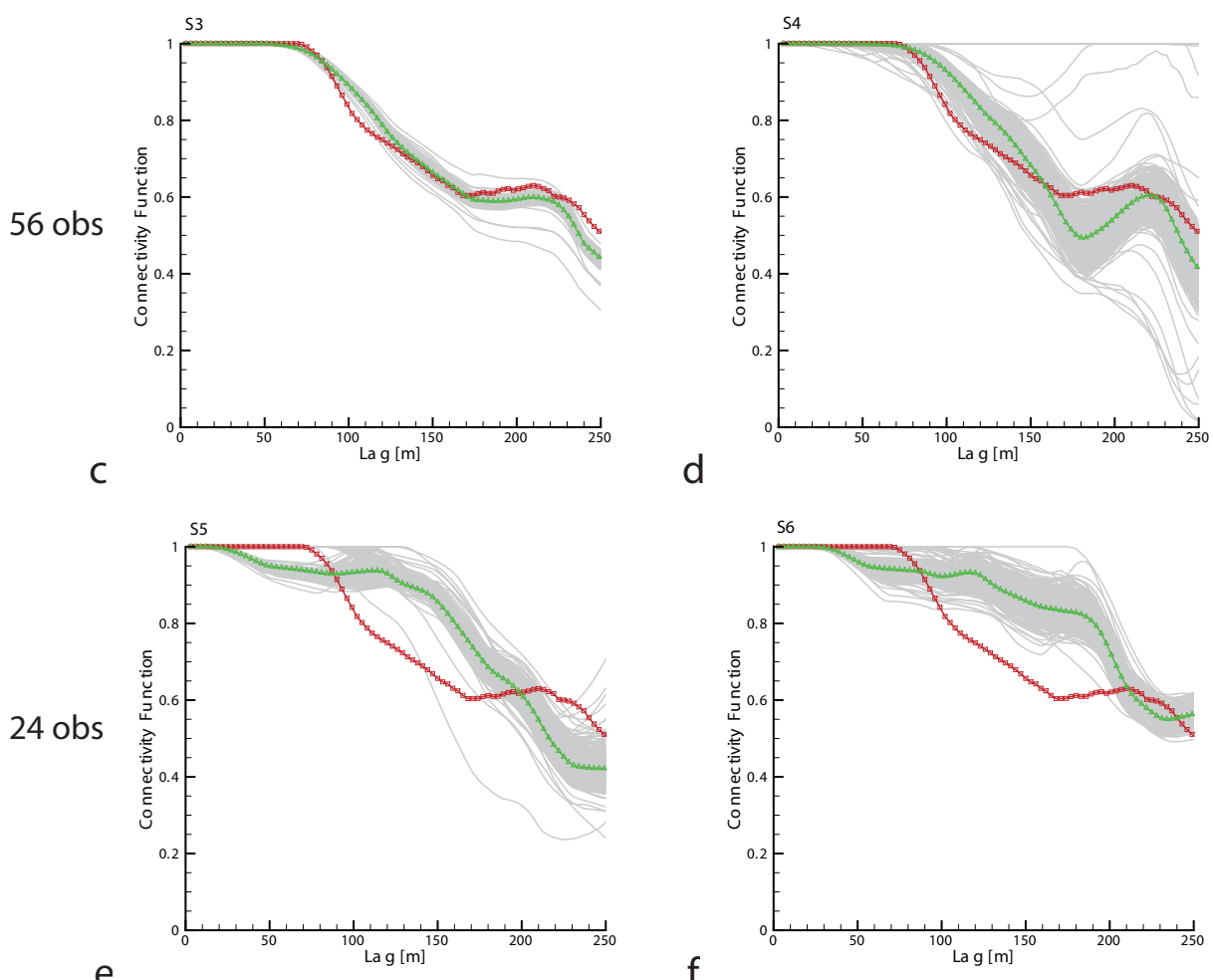

d

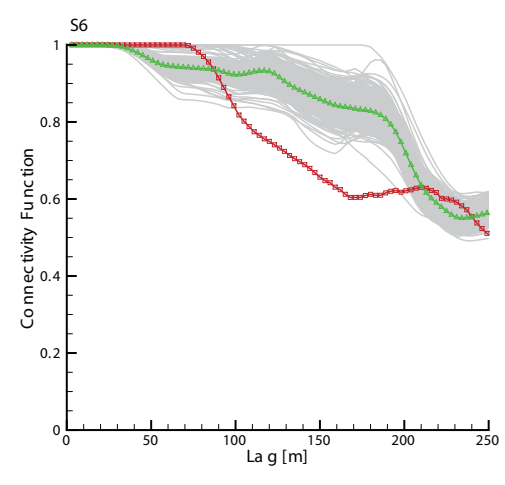

Figure 3.25. Scenarios S1-S6. Connectivity curves. The red square line corresponds to connectivity curves in the reference, the green delta line corresponds to the mean of the ensemble, and the gray lines correspond to the individual realizations 


\subsection{Summary and conclusion}

In this paper we wanted to show the power of transient piezometric head information for the characterization of the spatial variability of hydraulic conductivity, for hydraulic conductivity fields displaying spatial patterns that can not be characterized with multi-Gaussian approaches. We have taken an extreme position in that we assume that we do not have any information about hydraulic conductivity, neither locally nor globally, except for its bimodal marginal distribution. Zhou et al. (2011) already showed that transient piezometric head was enough for hydraulic conductivity characterization if a training image for the hydraulic conductivity was available. Our main finding is that without such a training image but with enough transient piezometric head information, we can generate an ensemble of realizations that captures the main patterns of the non-Gaussian reference field. The number of piezometers below which the characterization will deteriorate is very much problem dependent; both the type of underlying conductivity field and the boundary conditions of the flow problem will have an impact on how many piezometers are necessary and for how long they have to be measured. In this paper we do not seek to give an answer to this latter question, but rather emphasize that even for a clearly channelized bimodal conductivity field, the transient piezometric heads carry very valuable information about the conductivity spatial heterogeneity, and therefore, we should always do every attempt to try to assimilate these data into our flow models. On occasions, piezometric head is disregarded for the purpose of inverse modeling on the account that it is a low pass filter of the conductivities, it is true that in the examples shown, the assimilation of piezometric head cannot get the short scale variability of the reference field, but the main patterns are clearly identified. We took a rather radical approach, i.e., no spatial information was used. However, additional information about the patterns in conductivity, without the need of resorting to a training image, such as the main orientation of the channels and their width, will help improving the characterization. If in addition, a training image is available, the characterization would improve as demonstrated in the reference scenario S0.

We have also shown that filter inbreeding can be reduced with covariance inflation techniques. Although, when no inbreeding appears, as in scenario S0, there is no need for such an inflation.

We conclude that the NS-EnKF approach developed by Zhou et al. (2011) proves again capable of preserving the bimodality of the reference field, even for the case in which there is very limited information about the log-conductivities. Covariance localization and inflation are necessary to reduce filter inbreeding. For the specific case analyzed in this paper, 56 piezometers were enough to capture the main channels in the reference field; however, our purpose is not to give a rule about how many piezometers are 
needed, but rather to emphasize the importance of accounting for transient piezometric heads in our inverse modeling. 
to be submitted

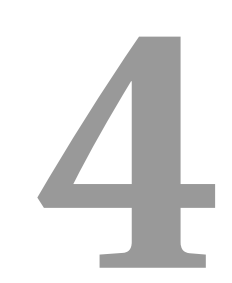

\title{
Probability Fields Revisited in the Context of Ensemble Kalman Filtering
}

\begin{abstract}
Hu et al. (2013) proposed an approach to update complex geological facies models generated by multipoint simulation while keeping geological and statistical consistency. Their approach is based on mapping the facies realization onto the spatially uncorrelated uniform random numbers used by the sequential multipoint simulation to generate the facies realization itself. The ensemble Kalman filter was then used to update the probability realizations, which were used to generate a new facies realization by multipoint sequential simulation. This approach has a poor performance that we attribute to the fact that, being the probabilities random and spatially uncorrelated, their correlation with the state variable (piezometric heads) is very weak, and the Kalman gain is always small. The approach is reminiscent of the probability field simulation, which also maps the conductivity realizations onto a field of uniform random numbers; although the mapping now is done using the local conditional distribution functions built based on a prior statistical model and the conditioning data. Contrary to $\mathrm{Hu}$ et al. (2013) approach, this field of uniform random numbers, termed a probability field, displays patterns of spatial correlation related to the correlation of the conductivities, and, therefore, the correlation between probabilities and state variable is as strong as the correlation between conductivities and state variable could be.
\end{abstract}


Similarly to $\mathrm{Hu}$ et al. (2013), we propose to use the ensemble Kalman filter to update the probability fields, and show that the correlation between probability values and state variables improves the performance.

\subsection{Introduction}

In the pursue of an application of the ensemble Kalman filter (EnKF) Evensen (1994, 2003) and multipoint simulation (MPS) Guardiano and Srivastava (1993b); Strébelle (2000) to assimilate dynamic production data into reservoir facies models, $\mathrm{Hu}$ et al. (2013) proposed a method based on the mapping of a given reservoir model onto the uniform random numbers used to generate it. Recall that MPS proceeds by visiting, using a predetermined path sequence, all nodes of a grid; computing the local probability of the parameter being simulated conditioned to the already simulated points (in MPS simulation this probability is deduced from a training image); and chosing a parameter value from this probability distribution by drawing a uniform random number between 0 and 1 . At each node, there is a uniform random number drawn, with each random number being independent of the rest. Therefore, given the parameters which will drive the MPS simulation, i.e., training image, size of search neighborhood to look for conditioning data, maximum number of conditioning data to retain, etc., there is a one-to-one mapping between a facies model and a spatially independent realization of uniform numbers. If the realization of uniform numbers is modified, a new geological model is obtained, which will be as geologically consistent with the training image as the first realization. The initial objective of the work by $\mathrm{Hu}$ et al. (2013) was to assimilate production data onto binary facies models; the mapping of the facies realization (a realization consisting only of only two numbers) onto uniform numbers has the additional interest of mapping a discrete field into a continuous one, which will be amenable of treating by the EnKF. The method proposed by Hu et al. (2013) simply applies the standard EnKF to the uniform numbers, instead of onto the facies values.

We have tested the method by Hu et al. (2013) in the context of assimilating piezometric heads in an aquifer and we have found that the method does not perform very well. We think that this underperformance is due to the very weak cross-correlation that there is between the uniform numbers and the state variables. Recall that the EnKF proceeds in two steps: forecast and analysis. The forecast step presents no problem, it is based in the solution of the numerical model appropriate to the process being studied. The analysis step is the one in which the approach by Hu et al. (2013) fails. In the analysis step, discrepancy between predicted and observed states at observation locations is used to update the parameters driving the stateequation. This update is proportional to the said discrepancy, but also to 
what is called the Kalman gain, which is a function of the auto- and crosscovariances of parameters and state variables. When the parameters being updated are uniform random numbers that are uncorrelated in space, the auto-covariance of the parameters and the cross-covariance are very weak, resulting in a very small Kalman gain. The net effect is that during the analysis step the update of the uniform random field is small and limited to a very narrow area around observation locations.

Mapping parameters onto probabilities reminded us of the probability field approach Froidevaux (1993) to generate conditional realizations of a given parameter using unconditional (but correlated) realizations of a uniform random field. In this case, the mapping uses the local conditional distribution functions of the parameter. Given a set of conditional data, and a set of structural parameters, one can obtain the local conditional distribution functions by simple kriging, indicator kriging, numerically from a training image, or from an ensemble of realizations generated otherwise. Once the local conditional distribution functions are defined at each point within the domain, there is a one-to-one mapping between any parameter realization and a probability field. The probability field does not have to be conditional to the parameter values since the conditioning will happen when reading back the (Heaviside) cumulative distribution function at conditioning locations; yet, it needs to be correlated, to preserve the correlation structure of the parameters. The interest of the probability field approach was the generation of conditional realizations (of the parameter) from unconditional realizations (of probabilties), which were, at the time, much cheaper to generate than conditional ones. The method never had a wide acceptance for the difficulty of establishing, a priori, which the correlation structure of the probabilities should be. Yet, there are some interesting applications of probability fields for inverse modeling (Capilla et al., 1999; Capilla and Llopis-Albert, 2009).

We have decided to revisit the concept of probability fields in the context of data assimilation by the EnKF. The spatial correlation of the probability fields will be determined a posteriori, from the ensemble of parameter realizations, thus avoiding the main problem of the original idea. We have applied the EnKF on the probability fields and found that the method gives good results.

The paper continues by presenting an extension to the algorithm by $\mathrm{Hu}$ et al. (2013) for the simulation of continuous variables, together with the implementation of EnKF using probability fields. Next, both algorithms are tested in a synthetic channelized aquifer with a bimodal histogram of conductivities. 


\subsection{Methodology}

In this section, we will describe the two algorithms. The algorithm based on $\mathrm{Hu}$ et al. (2013) work will be referred to as the Uncorrelated Probability Field (UPF) method, and the one based on Froidevaux (1993)'s approach will be referred as the Correlated Probability Field (CPF) method.

In both methods, the parameter which is updated by the EnKF is probability (which should follow a uniform distribution between 0 and 1 ); however, given that the EnKF is optimal when the parameters follow a Gaussian distribution, we will convert the uniform probabilities into Gaussian deviates and apply the EnKF to the latter. The conversion simply amounts to reading the standard $\mathcal{N}(0,1)$ Gaussian cumulative distribution function.

\subsubsection{Uncorrelated probability field method}

$\mathrm{Hu}$ et al. (2013) approach only updated the facies distribution and then assumed homogeneous parameters within each facies. We would like to have heterogeneous conductivities on top of the facies heterogeneity. For this reason, the generation of each conductivity realization is done in two steps, in the first step a facies realization is generated using MPS (we limit this analysis to two facies), and, in the second step, each facies is independently populated with conductivity values using sequential Gaussian simulation (SGS). This implies that the mapping between conductivities and probabilities requires not just one random field of uncorrelated uniform numbers, but three, one to generate de facies and two to generate the conductivities that will be associated with each facies. Conceptually the approach is the same as the original one: conductivities are mapped onto uncorrelated probabilities, and these probabilities are the parameters updated by the EnKF.

Figure 4.1 shows the flowchart for this method, which can be described as follows:

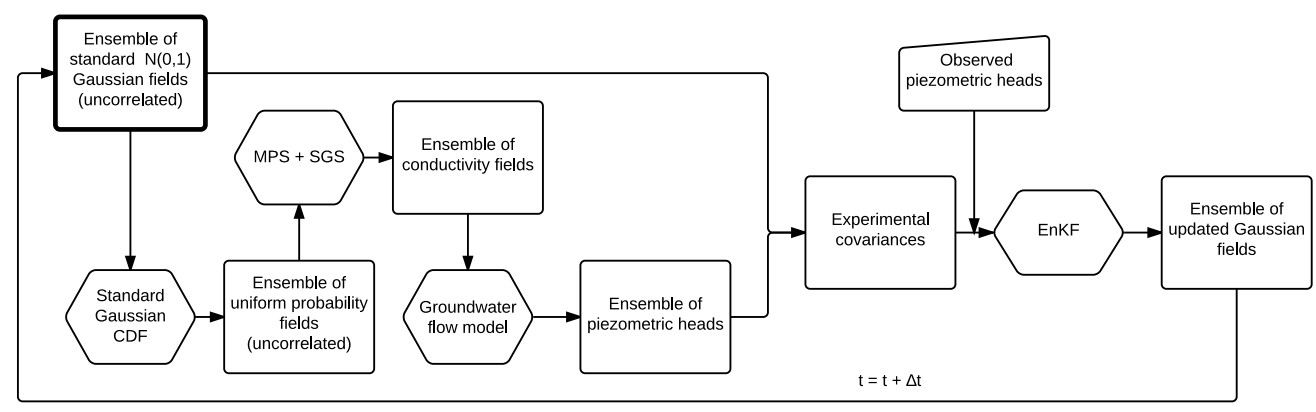

Figure 4.1. Work flow for the Unconditional Probability Field method. The starting step is highlighted in bold. 
1. Generate an ensemble of uncorrelated standard Gaussian fields $\mathcal{N}(0,1)$.

2. Transform the uncorrelated Gaussian deviates into uniform probability fields.

3. Use these uniform fields with MPS and SGS to generate an ensemble of conductivity fields.

4. Feed the conductivity fields into the groundwater flow model, with appropriate initial and boundary conditions, and given sinks and sources; as a result, we obtain an ensemble of piezometric heads.

5. Compute the auto- and cross-covariances of the Gaussian deviates obtained in step 1 and the piezometric heads of the previous step.

6. Sample the piezometric heads at the observation points.

7. Update the Gaussian deviates by the EnKF and return to step 1.

\subsubsection{Correlated probability field method}

For the CPF method we need to establish first which are the local conditional probability distributions given the conditioning data. When the probability field method was developed, these probability distributions were obtained by kriging: simple kriging when the random function model was multiGaussian, indicator kriging when the model was non-parametric and based on the indicator covariances. With the advent of multipoint statistics, model statistics are not specified analytically but inferred from a training image; the training image can also be used to define the local conditional probability distributions. An alternative, used in this work, is to infer the conditional distributions from an ensemble of realizations that has been generated by whichever stochastic simulation approach.

Before the CPF method starts, we have to generate an initial ensemble of conductivity realizations from which to compute the local conditional distribution function at each grid node. (When there are no conditioning data, the local conditional distribution coincides with the marginal one everywhere.)

Considering that the map of local distribution functions has been already determined determined one way or another, the flow chart of the CPF is shown in Figure 4.2, which can be described as follows:

1. Transform the ensemble of conductivity fields into an ensemble of (correlated) probability fields by replacing each conductivity value with the probability associated to its local conditional distribution.

2. Transform the probabilities into Gaussian deviates using an inverse standard Gaussian cumulative distribution function $\mathcal{N}(0,1)$. 


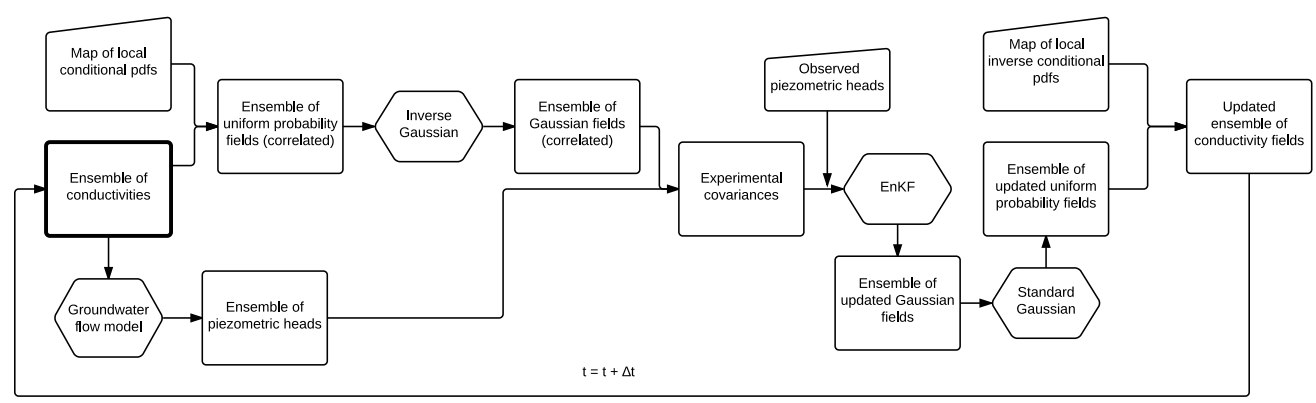

Figure 4.2. Work flow for the Conditional Probability Field method. The starting step is highlighted in bold.

3. Feed the conductivity fields into the groundwater flow model, with appropriate initial and boundary conditions, and given sinks and sources; as a result, we obtain an ensemble of piezometric heads.

4. Compute the auto- and cross-covariances of the Gaussian deviates obtained in step 2 and the piezometric heads of the previous step.

5. Sample the piezometric heads at the observation points.

6. Update the Gaussian deviates by the EnKF.

7. Transform the Gaussian deviates into updated probabilities using the standard Gaussian cumulative distribution function.

8. Transform back the updated probability fields into updated conductivities through the inverse local conditional distribution and return to step 1.

\subsection{Synthetic Example}

The performance of the two methods will be evaluated on a synthetic confined aquifer of $50 \mathrm{~m}$ by $50 \mathrm{~m}$ by $5 \mathrm{~m}$, discretized into 50 by 50 by 1 cells. The aquifer is composed of $35 \%$ high conductivity sand and $65 \%$ low conductivity shale. The spatial heterogeneity of the sand/shale distribution is characterized by the training image shown in Figure 4.3 (replicated after (Strebelle, 2002)). Hydraulic log-conductivity within the sand follows a Gaussian distribution with mean of $2.3 \ln (\mathrm{m} / \mathrm{d})$ and a standard deviation of $1 \ln (\mathrm{m} / \mathrm{d})$, while in shale has a mean of $-3.5 \ln (\mathrm{m} / \mathrm{d})$ and a standard deviation of $0.6 \ln (\mathrm{m} / \mathrm{d})$. The conductivity in sand has a spatial correlation anisotropy characterized by an exponential variogram with ranges of $48 \mathrm{~m}$ in the horizontal direction and 24 in the vertical direction; while conductivity in shale displays an isotropic correlation characterized by an exponential 
Table 4.1. Parameters of the random functions describing the spatial continuity of the sand and shale log-conductivities

\begin{tabular}{lccccccc}
\hline Facies & Proportion & $\begin{array}{c}\text { Mean } \\
{[\ln [\mathrm{m} / \mathrm{d}]]}\end{array}$ & $\begin{array}{c}\text { Std.dev } \\
{[\ln [\mathrm{m} / \mathrm{d}]]}\end{array}$ & $\begin{array}{c}\text { Variogram } \\
\text { type }\end{array}$ & $\begin{array}{c}\lambda_{x} \\
{[\mathrm{~m}]}\end{array}$ & $\begin{array}{c}\lambda_{y} \\
{[\mathrm{~m}]}\end{array}$ & sill \\
\hline Sand & 0.35 & 2.3 & 1.0 & exponential & 48 & 24 & 1 \\
Shale & 0.65 & -3.5 & 0.6 & exponential & 24 & 24 & 0.35 \\
\hline
\end{tabular}

variogram with range of $24 \mathrm{~m}$. These parameters are summarized in Table 4.1 .

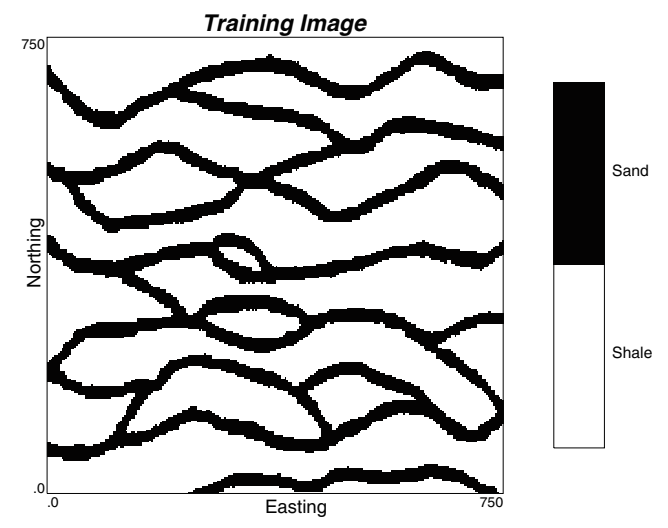

Figure 4.3. Training image

The log-conductivities in the synthetic aquifer are built in two steps. In the first step, using the code SNESIM Strebelle (2002), a binary sand/shale realization is generated coherent with the training image in Figure 4.3. (Note that the training image extends over a much larger area than the aquifer.) In the second step, using the code GCOSIM3D Gómez-Hernández and Journel (1993), each facies is populated independently with log-conductivity values generated by sequential Gaussian simulation. The resulting field and its histogram are shown in Figure 4.4. The synthetic aquifer displays the channelized structure of the training image and has a bimodal distribution with global mean of $-1.1 \ln (\mathrm{m} / \mathrm{d})$ and global standard deviation of $2.8 \ln (\mathrm{m} / \mathrm{d})$.

A transient groundwater flow problem is solved in the synthetic aquifer using MODFLOW (e.g., McDonald and Harbaugh, 1984; Harbaugh et al., 2000). The top and bottom boundaries are impermeable, the left boundary has a prescribed head equal to $8 \mathrm{~m}$, and the right boundary has prescribed pumping at the segments coinciding with the sand channels with a total pumping of $190 \mathrm{~m}^{3} / \mathrm{d}$ distributed as shown in Figure 4.4. The initial head is uniform and equal to $8 \mathrm{~m}$ throughout. The specific storage is homogeneous 

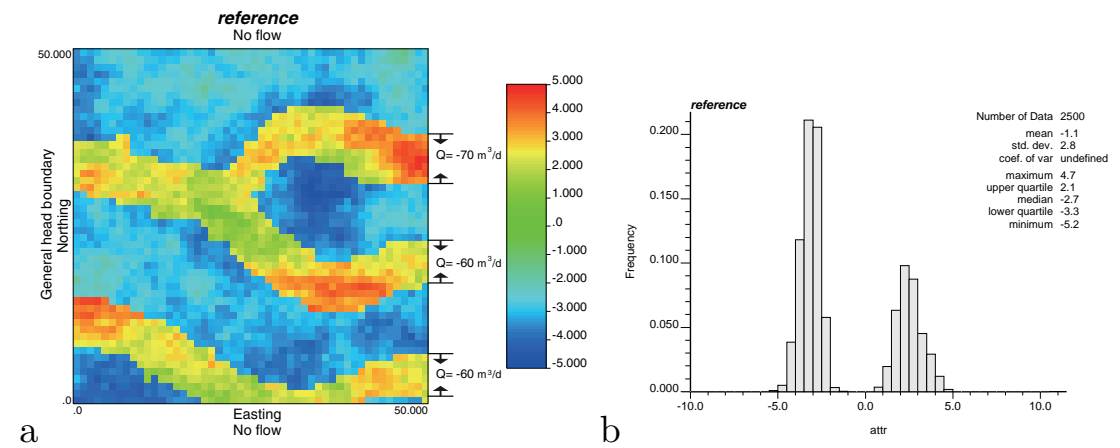

Figure 4.4. Synthetic aquifer and log-conductivity histogram.

en equal to $0.1 \mathrm{~m}^{-1}$. The total simulation time is $500 \mathrm{~d}$, discretized into 100 time steps following a geometric sequence with ratio 1.05. (The first time step is 0.19 days.)

After solving groundwater flow, the piezometric heads at the 25 points shown in Figure 4.5 are recorded and saved for all time steps. The data from the first 60 time steps ( 67 days) will be used for assimilation by the EnKF. No facies data, or log-conductivity data are used.

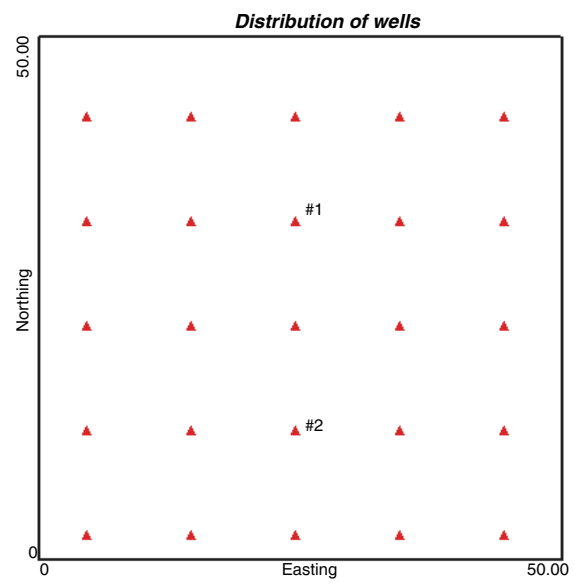

Figure 4.5. Distribution of observation piezometers

Both the UPF and the CPF begin with an ensemble of realizations that will be progressively updated by ensemble Kalman filtering with each time step. In this work, we generate 800 realizations, using the same two-step approach as for creating the synthetic aquifer, that is, we use the same training image for the facies realizations by MPS and the same parameters of Table 4.1 to fill in the facies with log-conductivities. These realizations are unconditional since no data on facies or log-conductivity are available. 
For the purpose of applying the UPF method, we have recorded the uniform random numbers used for the generation of the fields; then, these uniform random numbers are transformed into Gaussian deviates using the inverse of the standard Gaussian distribution. The UPF starts from this ensemble of realizations of Gaussian deviates (see Figure 4.2).

For the purpose of applying the CPF method, we have computed, at each node, the local conditional distribution function, which, for this case, since the realizations are unconditional, coincides with the one derived from the global histogram of the reference (see Figure 4.4b).

Next, we evaluate the ability of both methods to reproduce the patterns observed in the synthetic aquifer after assimilating the piezometric heads for the first 60 time steps. We will also evaluate the ability of both methods to reproduce the observed piezometric heads.

\subsection{Reproducing log-conductivity}

Figure 4.6 shows the ensemble mean and the ensemble variance of the initial ensemble of log-conductivity realizations used in both approaches, together with the global histogram. The 800 realizations were generated unconditional and, consequently, their ensemble mean and ensemble variance are flat and equal to their marginal values.

Figures 4.7 shows the ensemble mean, ensemble variance and global histograms computed for both methods after assimilating piezometric head data for 10 time steps. Figure 4.8 shows similar results after 60 time steps. From these two figures it is evident that the CPF outperforms the UPF. The ensemble mean of the log-conductivities obtained by the CPF already delineates the channels observed in the synthetic aquifer, and the ensemble variance highlights that there is some residual uncertainty at the channel edges. Whereas, the ensemble mean and variance of the log-conductivities obtained by the UPF are a little bit more informative than the mean and variance for the initial ensemble, yet, they are quite far from the results achieved by the CPF.

Notice that the bimodal histogram of the log-conductivities is respected by the ensemble of updated realizations in both methods. In the case of the UPF this happens by construction, since, independently of the updated probabilities resulting from the application of the EnKF, the associated log-conductivities are fully consistent with the prior statistical model since they are obtained by MPS followed by SGS with fixed training image and statistical parameters. In the case of the CPF it depends on the histogram of the probabilities, when this histogram remains uniform; then, sampling back the local conditional distributions will result in a global histogram respecting the prior one. 
a

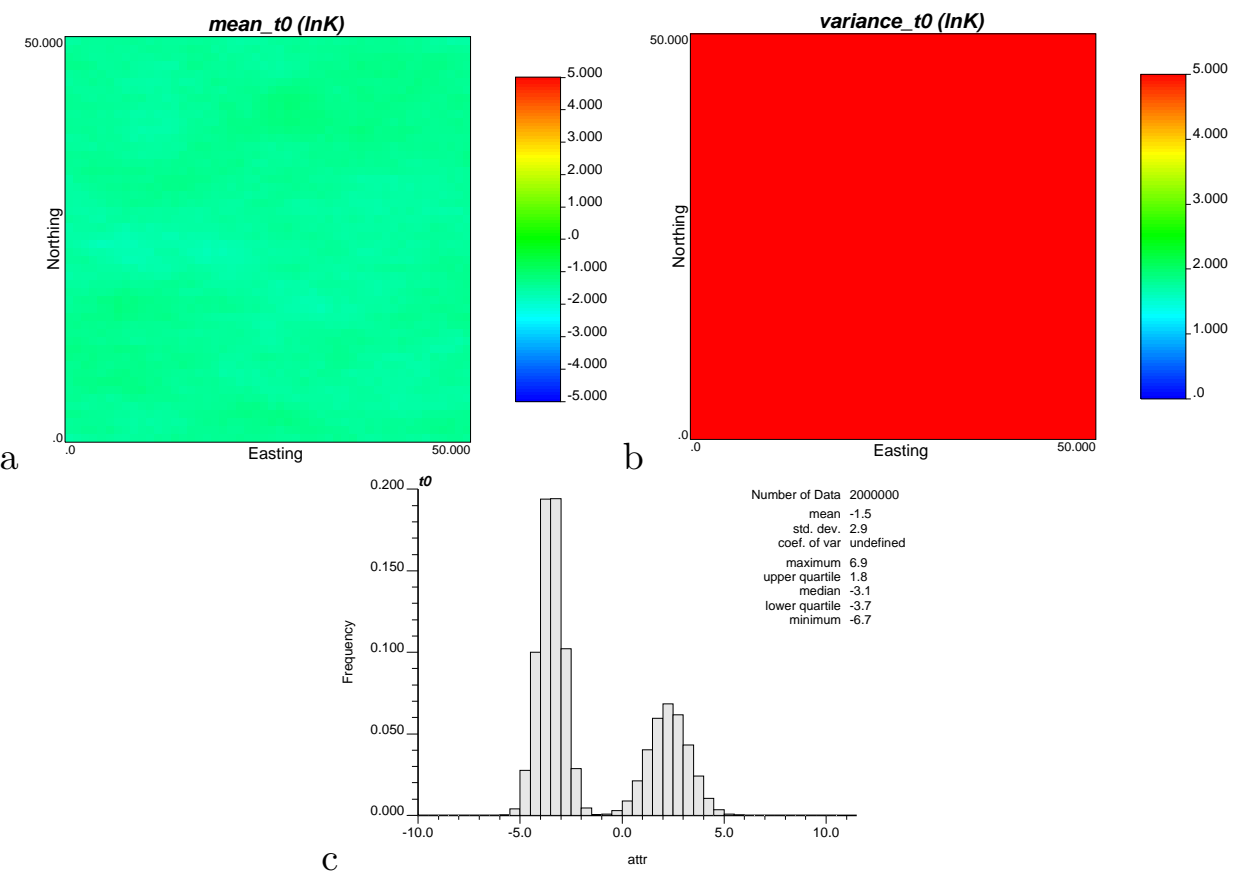

Figure 4.6. Ensemble mean, ensemble variance and ensemble histogram of the initial ensemble of log-conductivity realizations.

To try to explain why the dramatic difference in performance we will show the evolution of realizations number 400 and 800 at different times during the assimilation process. Figures 4.9 and 4.10 show these two realizations at the beginning (same realization for both approaches) and after time steps $9,10,59$ and 60 . The objective of this display is to show the changes that can occur in a single update step (between time steps 9 and 10 or between time steps 59 and 60) and the overall evolution. In the UPF, the changes between consecutive time steps can be quite drastic, given the nature of the sequential simulation algorithms, in which each node is simulated based on the previously simulated nodes; however, since each realization is obtained by MPS and SGS, the delineation of the facies (as in the training image) is quite clear at all steps. In the $\mathrm{CPF}$, the changes between consecutive time steps are quite smooth, the EnKF updates smoothly the probabilities, and when reading back the updated probabilities through the local distribution functions the changes are smooth, too; however, the controls that generated the facies in the initial realizations disappear, and the delineation of the facies becomes fuzzier than in the UPF, yet the look of the final updated realizations obtained with the CPF is closer to the reference than with the UPF. 


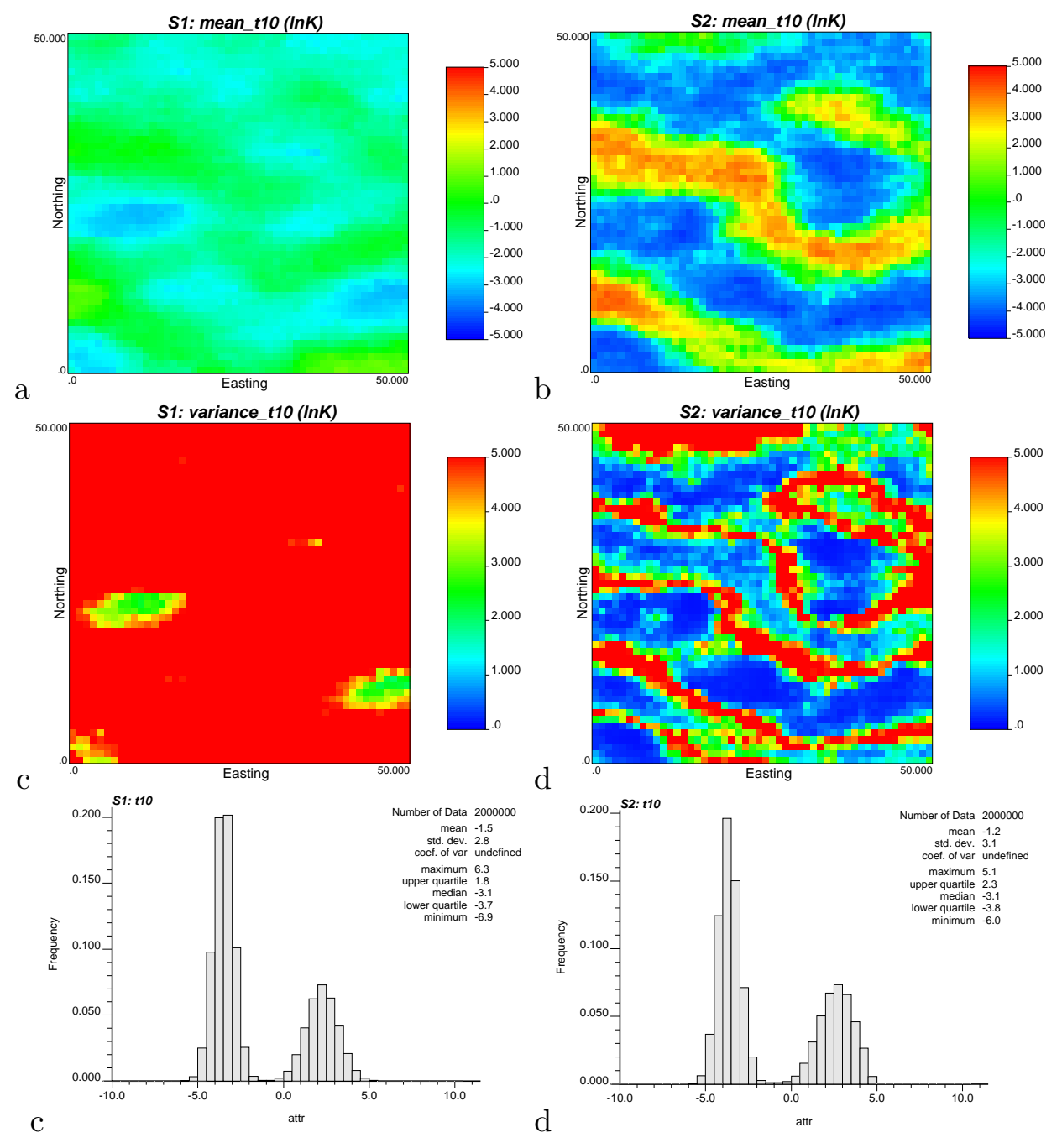

Figure 4.7. Ensemble mean, ensemble variance and ensemble histogram of the log-conductivity realizations obtained after 10 assimilation time steps. Left column, uncorrelated probability fields (S1). Right column, correlated ones (S2). 
a

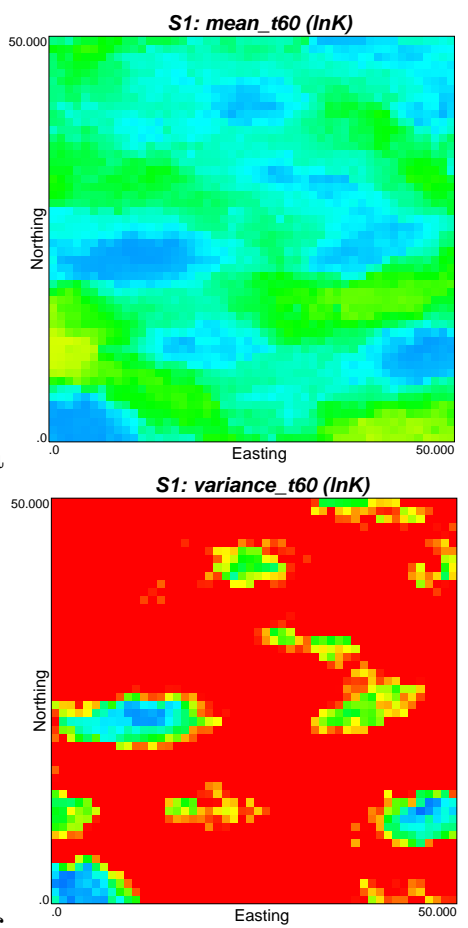

$\mathrm{c}$

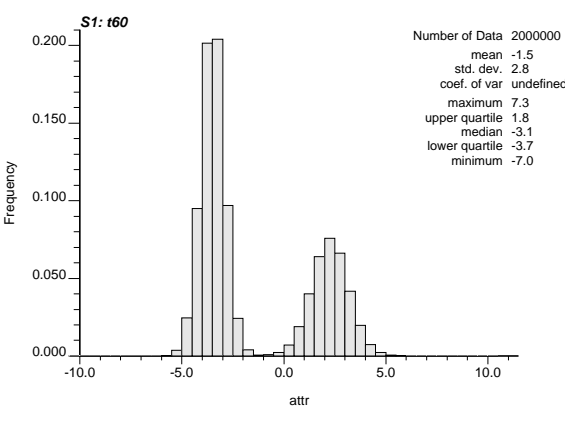

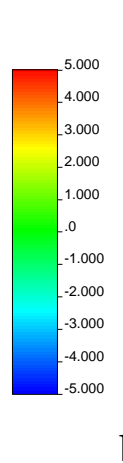

$\mathrm{b}$

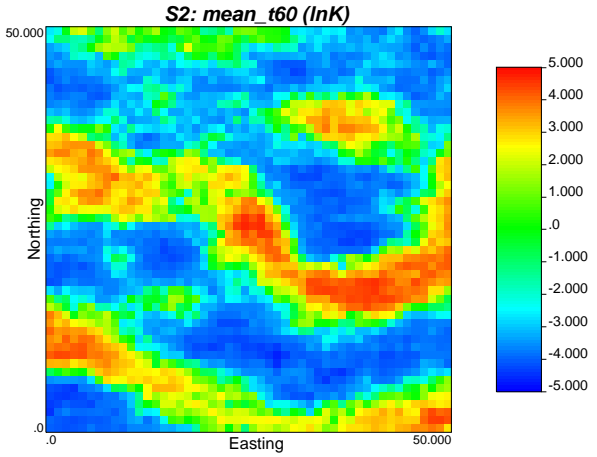

S2: variance_t60 (InK)

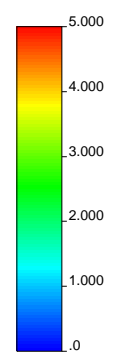

d
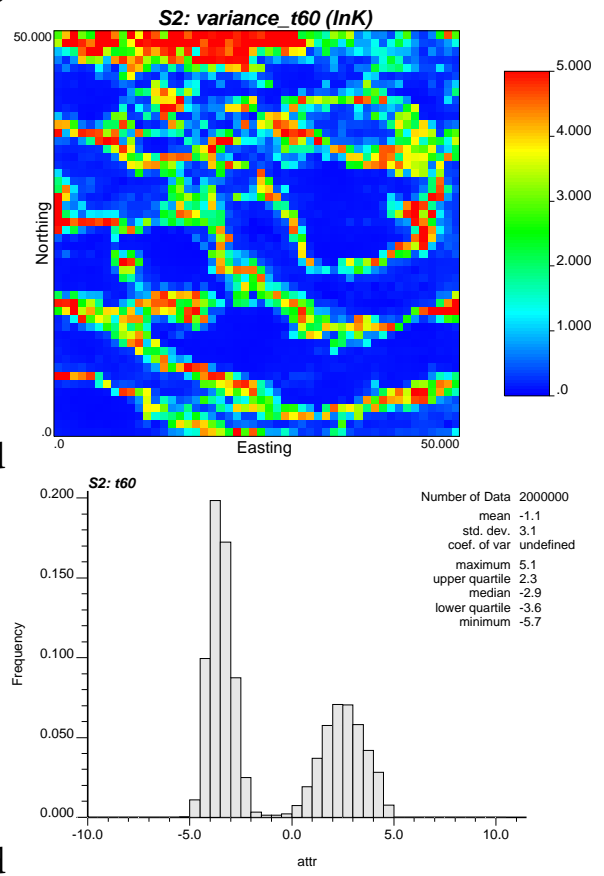

Figure 4.8. Ensemble mean, ensemble variance and ensemble histogram of the log-conductivity realizations obtained after 60 assimilation time steps. Left column, uncorrelated probability fields (S1). Right column, correlated ones (S2). 


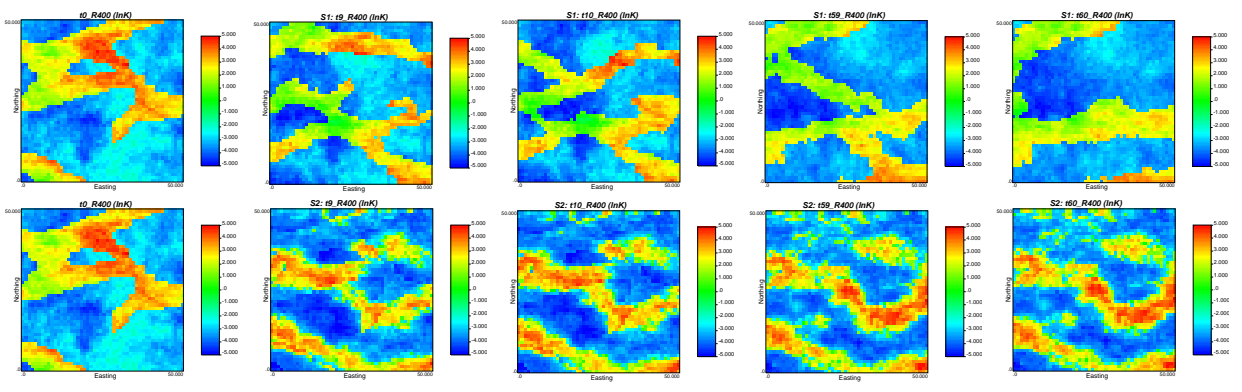

Figure 4.9. Evolution of the 400th realization in both methods. Top row, uncorrelated probabilities (S1); bottom row, correlated probabilities (S2). Columnwise from left to right, initial realization (same for both approaches), after time step 9, 10,59 and 60 .

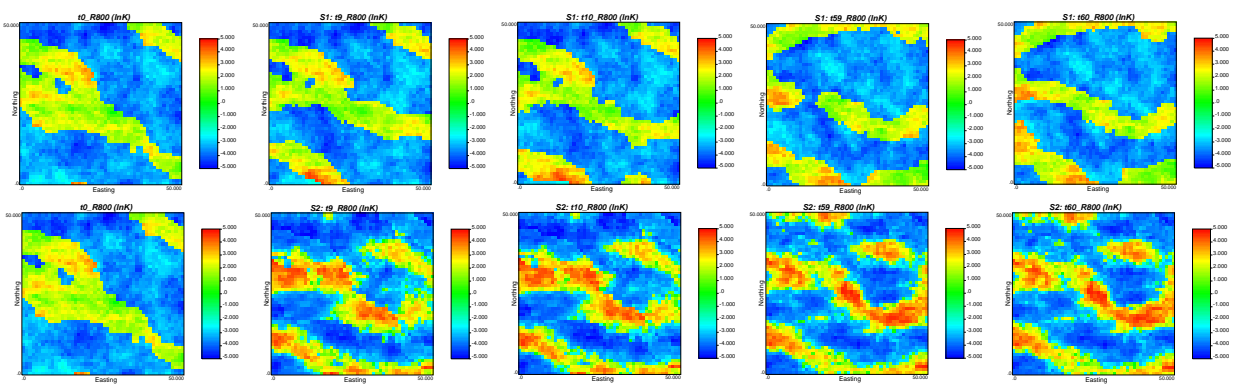

Figure 4.10. Evolution of the 800th realization in both methods. Top row, uncorrelated probabilities (S1); bottom row, correlated probabilities (S2). Columnwise from left to right, initial realization (same for both approaches), after time step 9, 10,59 and 60 . 
It is interesting to analyze how the update in the log-conductivity field relates to the update of the underlying probability fields. Figure 4.11 shows the update of the underlying Gaussian deviates at time steps 10 and 60 as computed by the EnKF in realization 400 for both methods. In the case of the UPF, there are three Gaussian deviate fields, we are showing only the field that is used to update the facies distribution. Similar results are shown for realization 800 in Figure 4.12. The update is the result of the product of the Kalman gain by the discrepancy between predicted and observed piezometric heads. In the UPF, the updates of the Gaussian deviates are very local and short correlated, as a result of the very weak correlation between the uncorrelated probability fields and the piezometric heads; however, this local, random looking update of the probabilities produces changes in the facies quite important. Figure 4.13 shows the facies change in realizations 400 and 800 at time steps 10 and 60. Apparently, similar random updates of the Gaussian deviates induce quite different updates in the facies realization for realizations 400 and 800. This "discrepancy" between the Gaussian update and the facies update is due to the nature of the MPS and SGS sequential simulation algorithms, which can be regarded as chaotic. On the contrary, in the CPF, the updates in the Gaussian deviates are smoother that in the previous method and its magnitude reduces significantly as time proceeds. This reduction in the magnitude of the updates is the result of the piezometric head assimilation which in turn reduces the ensemble variance. 

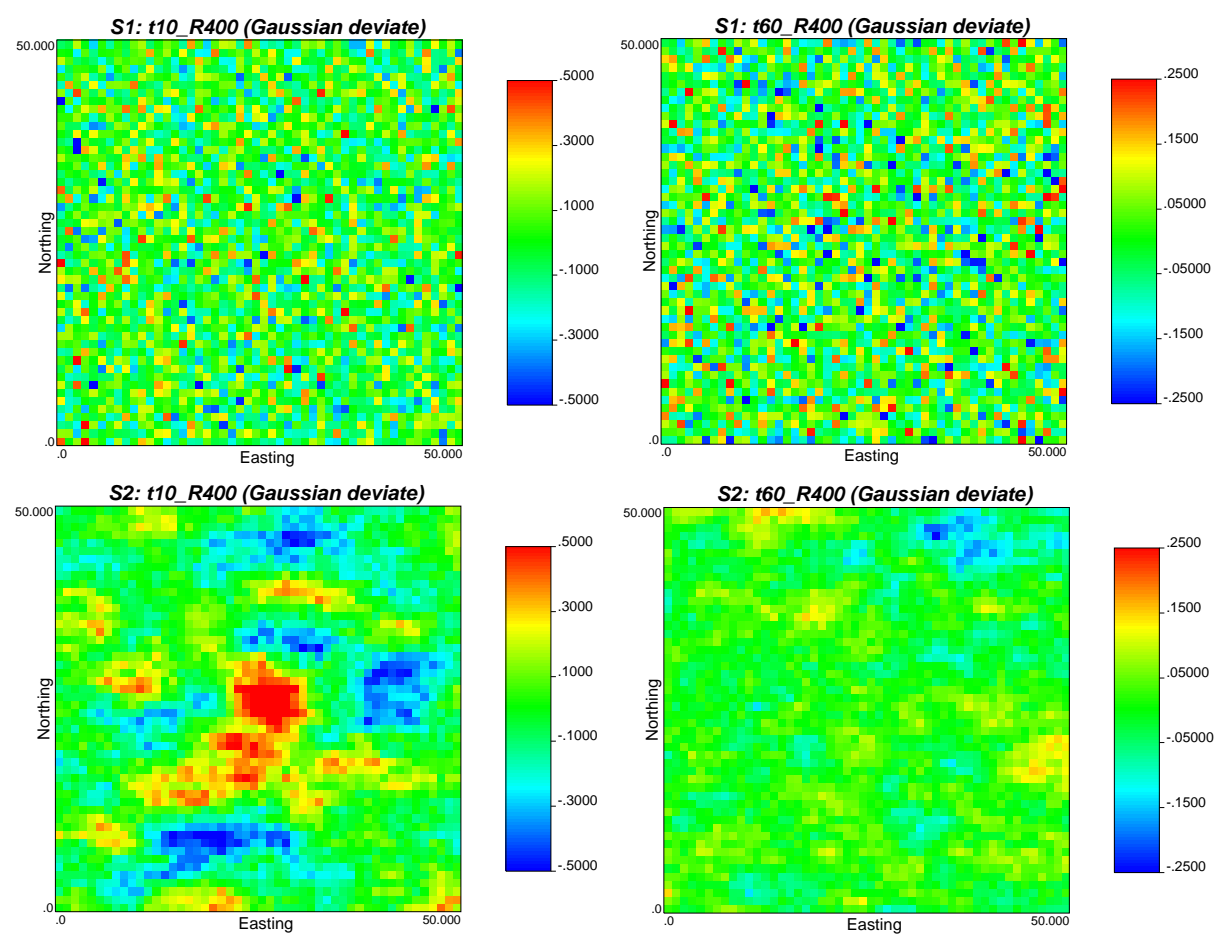

Figure 4.11. Increment of Gaussian deviates in realization 400. Top row, uncorrelated probabilities (S1); bottom row, correlated probabilities (S2). Left column, update at the 10th step; right column, update at the 60th step. 

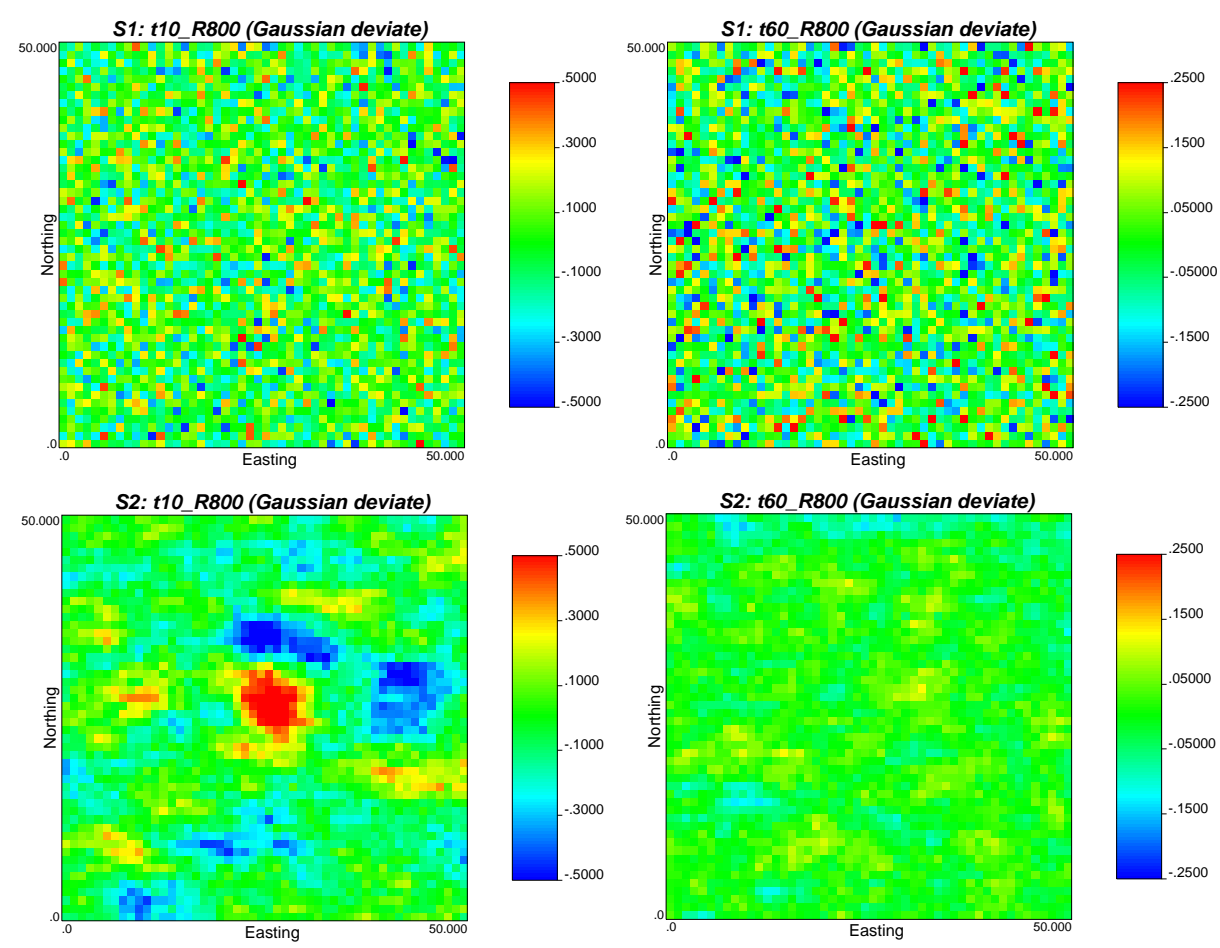

Figure 4.12. Increment of Gaussian deviates in realization 800. Top row, uncorrelated probabilities (S1); bottom row, correlated probabilities (S2). Left column, update at the 10th step; right column, update at the 60th step. 

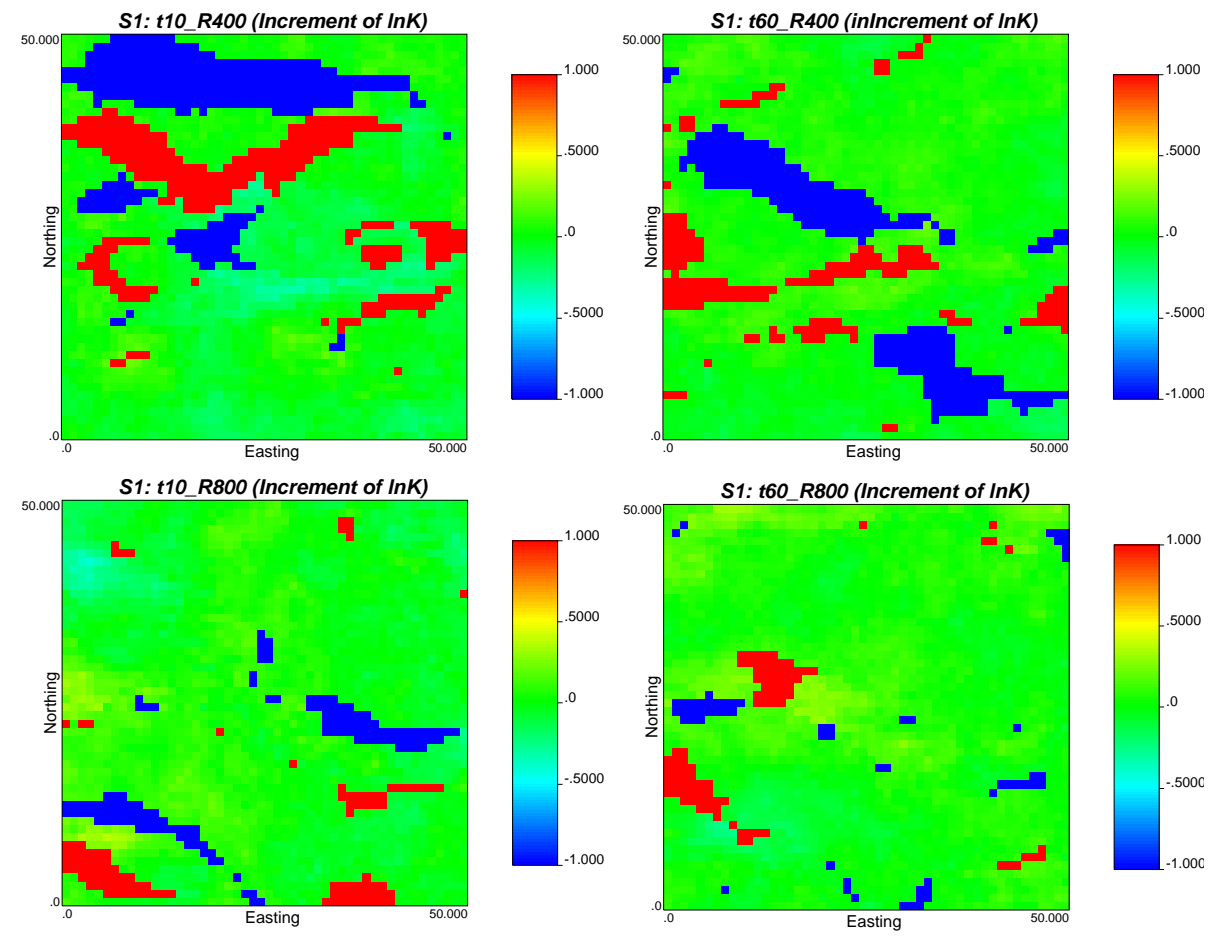

Figure 4.13. Faces changes induced by updating the probabilities in the uncorrelated probability field method. Blue means change from sand to shale, red means change from shale to sand, and green means no change. Right column for the updates at time step 10, left column for the updates at time step 60, top row for realization 400 , bottom row for realization 800 . 


\subsection{Reproducing piezometric head}

Figure 4.14 shows the piezometric head evolution at piezometers \#1 and \#2 of Figure 4.5 computed on the initial set of realizations. As expected, and given that the initial realizations are unconditional, their response to the groundwater flow conditions in the synthetic aquifer is quite variable among the realizations.
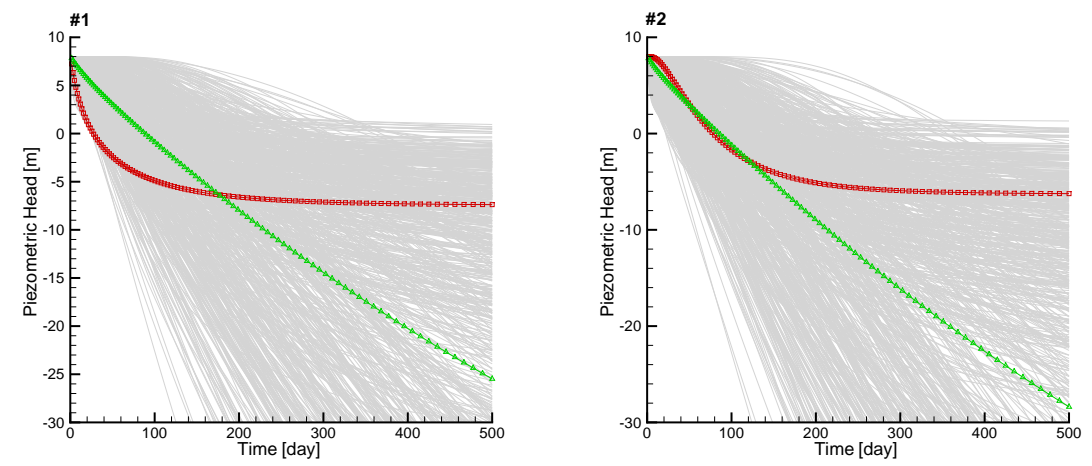

Figure 4.14. Reproduction of the observed piezometric head at the piezometers \#1 and \#2 of Figure 4.5 by the log-conductivity fields obtained after 60 time steps. Left, piezometer \#1; right, piezometer \#1. The red squares correspond to the head evolution in the synthetic aquifer, the gray lines are the head evolution in the individual realizations, and the green triangles correspond to the ensemble mean.

Figure 4.15 shows the piezometric head evolution at the same piezometers for the two methods after 60 assimilation time steps. The UPF shows a minor improvement with respect to the initial realizations, whereas the $\mathrm{CPF}$ is able to generated log-conductivity realizations capable of matching almost perfectly the observed piezometric heads.

Again, the poor behavior of the UPF must be attributed to the weak correlation between the Gaussian deviates and the piezometric heads in the UPF, plus the chaotic behavior of the sequential simulation algorithms: a small change in a single probability value could induce a very large change in the final log-conductivity map, particularly if this change happens in a node that is generated early in the path that visits all nodes being simulated. 

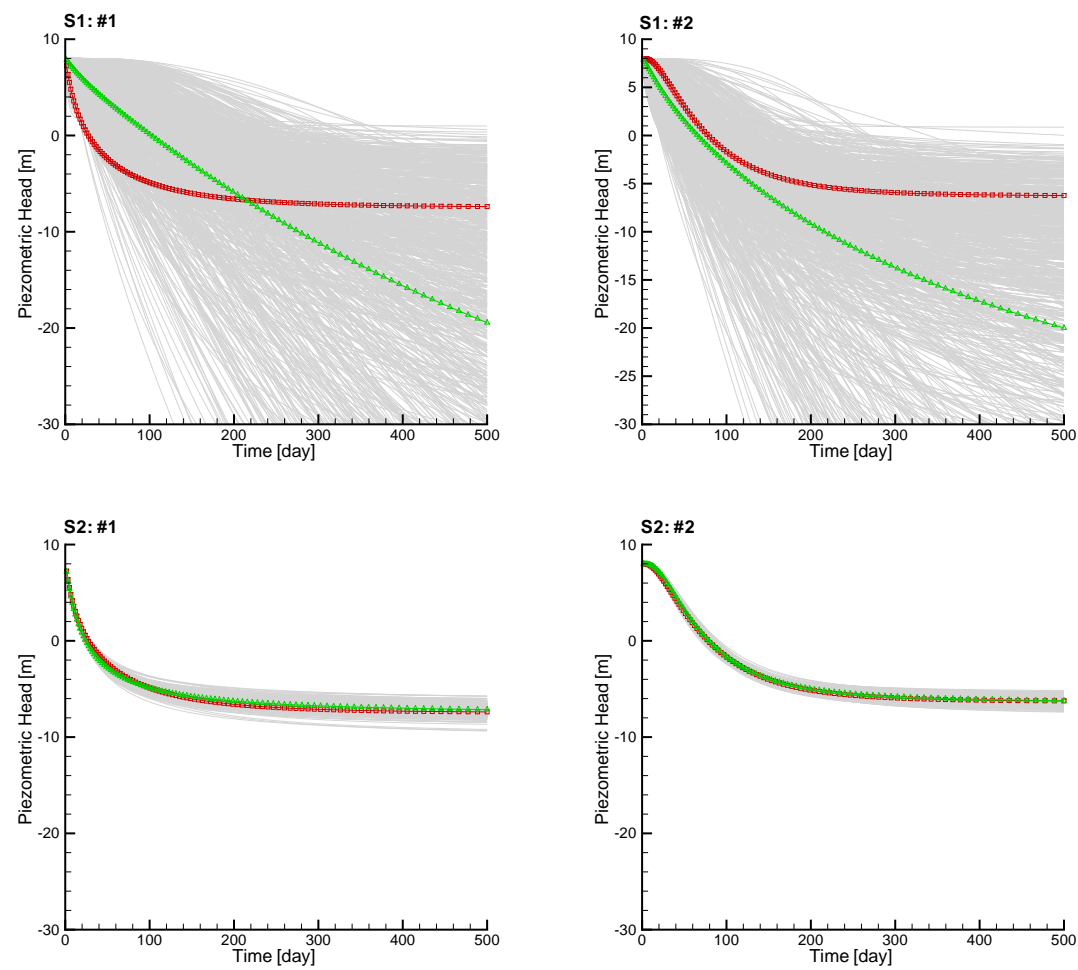

Figure 4.15. Reproduction of the observed piezometric head at the piezometers \#1 and \#2 of Figure 4.5 by the log-conductivity fields obtained after 60 time steps for both methods. Top row, uncorrelated probability field method (S1); bottom row, correlated probability field method (S2). Left, piezometer \#1; right, piezometer \#1. Meaning of lines same as previous figure. 


\subsection{Discussion}

The idea of mapping the conductivities onto probabilities was not new, but choosing as probabilities the ones used in the sequential simulation algorithm to draw from the local distribution functions conditioned to all previously simulated values was certainly a new idea. By modifying these probability fields and using them in a multipoint simulation, we can assure that the final realizations will always be coherent with the training image chosen. Therefore, it seemed a good idea to try to update the probability fields, instead of the conductivities directly in the context of the EnKF, in order to apply the EnKF to the generation of clearly non-Gaussian realizations. However, as it has been shown, the method has definite flaws linked precisely to the mapping procedure: on one hand, the probabilities are spatially uncorrelated and display a weak correlation with the state variables, on the other hand, the transformation of the perturbation of the probabilities onto perturbations of conductivities is chaotic, with small probability perturbations possibly inducing very large and widespread perturbations in conductivity.

Revisiting the probability field approach, which is based also in the mapping of conductivities onto probabilities, and formulating the EnKF method in terms of these probabilities, proves to be a powerful approach to generate conductivity realizations which display features difficult to model with multiGaussian-based approaches. This is an approach that should be reconsidered for data assimilation in hydrogeology and petroleum engineering. 
Water Resources Research, under review.

\title{
Inverse sequential simulation: A new approach for the characterization of non-Gaussian hydraulic conductivity fields
}

\begin{abstract}
Inverse sequential simulation (iSS) is a new inverse modeling approach based on sequential simulation for the characterization non-Gaussian hydraulic conductivity fields. The approach is described and demonstrated in a synthetic aquifer, and compared against the normal score ensemble Kalman filter (NS-EnKF). The new approach uses the sequential simulation paradigm to generate realizations borrowing from the ensemble Kalman filter approach the idea of using the experimental non-stationary cross-covariance between conductivities and piezometric heads computed on an ensemble of realizations. The resulting approach is fully capable of retrieving the main patterns of the reference field after conditioning on the piezometric heads.
\end{abstract}




\subsection{Introduction}

The quality of a groundwater model, particularly when studying the fate and transport of contaminants, relies very much on the quality of the characterization of hydraulic conductivities. Many studies have shown that, unless the heterogeneity of hydraulic conductivity is well captured in the groundwater model, the resulting transport predictions could be totally wrong; for instance, Gómez-Hernández and Wen (1994) show the high impact that properly accounting for heterogeneity has in transport predictions. But not only it is important to account for heterogeneity, as important is using the most adequate heterogeneity model. For many years, the only model consider for the spatial variability of hydraulic conductivity was the mutiGaussian model of log-conductivity, until it was recognized that the spatial patterns often observed in the subsurface (i.e., channels, permeability barriers, high conductivity streaks) were better modeled using alternatives to the multiGaussian model (Gómez-Hernández and Wen, 1998). Since then, there have been many attempts to define non multiGaussian random functions capable of capturing the spatial features difficult to capture by the multiGaussian ones, and then, to build algorithms for the spatial representation of hydraulic conductivity according to these new random function models.

Probably the most successful approach for the generation of realistic hydraulic conductivities is the one based on training images and multiple point statistics (Guardiano and Srivastava, 1993a; Strébelle, 2000; Strebelle, 2002). The next challenge was how to use these random functions in inverse modeling, that is, how to generate realizations of hydraulic conductivity that not only are consistent with the training image and conditional to the local measurements, but also that are inverse conditioned onto observed measurements of the state variables, such as piezometric head or solute concentration. Inverse modeling in hydrogeology and petroleum engineering has a long tradition (see Zhou et al. (2014) for a review) but, again, most inverse models rely on the assumption that hydraulic conductivity follows a multiGaussian model. Recent attempts to couple inverse approaches and non multiGaussian random functions have been attempted by Sun et al. (2009); Sarma and Chen (2009); Jafarpour and Khodabakhshi (2011); Hu et al. (2012b); Zhou et al. (2011, 2012); Attia and Sandu (2014), among others, with different degrees of success. The common denominator of all these proposals is that they are variants of algorithms that work for multiGaussian fields. In this paper we propose a completely new algorithm that is the result of blending some of the ideas underlying multivariate Gaussian sequential simulation (Gómez-Hernández and Journel, 1993) and ensemble Kalman filtering (Evensen, 2003). We have called this new algorithm inverse sequential simulation (iSS).

The algorithm proposed aims at the characterization of hydraulic conductivity using sparse observations of hydraulic conductivity, and sparse 
observations of piezometric heads. As a benchmark, the algorithm will be compared with the normal-score ensemble Kalman filter (NS-EnKF) (Zhou et al., 2011), which is one of the algorithms that best performs for nonGaussian inverse modeling. The paper continues with a description of the algorithm, followed by the comparison between NS-EnKF and iSS, and ends with some discussion and conclusions.

\subsection{Methodology}

The new algorithm is a breed of sequential simulation and the normalscore ensemble Kalman filter. On one hand, we borrow, from the ensemble Kalman filter (EnKF) (van Loon et al., 2000; Evensen, 2003; Blöschl et al., 2008; Karri et al., 2014), the idea of using an ensemble of realizations to compute an experimental, non-stationary conditional cross-covariance between conductivities and piezometric heads, and also the non-stationary conditional auto-covariances of both conductivity and piezometric head. On the other hand, we borrow, from the normal-score ensemble Kalman filter (Zhou et al., 2011), the idea of performing a normal-score transformation and thus work with a marginally Gaussian multivariate random function. We are aware that a normal-score transformation only produces marginallydistributed Gaussian variables, never multiGaussian ones; however, it has been shown in the normal-score EnKF that this transformation is quite effective in capturing non-Gaussian patterns. Finally, we use standard multivariate sequential Gaussian conditional simulation (Gómez-Hernández and Journel, 1993; Friedel and Iwashita, 2013) to generate realizations of the normal scores of conductivity conditioned to the normal scores of conductivity and to the piezometric head measurements. The state equation relating conductivity and piezometric heads, with its initial conditions, boundary conditions and forcing terms, is indirectly included in the sequential simulation through the conditional auto- and cross-covariances that are computed on the ensemble of realizations. When and if new head measurements are taken, the generated ensemble of conductivity realizations are used to forecast an ensemble of head realizations, new experimental covariances are computed, and a new ensemble of conductivity realizations is generated conditioned to the new head measurements.

The iSS method has been developed for its application under transient conditions, with a regeneration of the ensemble of conductivity fields each time new piezometric heads are measured. Consider that piezometric heads are collected sequentially in time. The method starts with an ensemble of conductivity fields generated according to a given random function model for the generation of this initial set no information about piezometric heads is used, this initial set should be conditional to conductivity measurements and other soft information such as geophysical data, when available. Then, 
for each time step for which piezometric heads are observed, the algorithm carries out the following: (i) an ensemble of piezometric head realizations are predicted on the basis of the last ensemble of conductivity fields by means of a numerical flow model, (ii) the conductivity and heads autoand cross-covariances are computed from the ensemble of realizations these covariances will be non-stationary, (iii) using a sequential multivariate simulation algorithm, a new ensemble of conductivity fields conditioned to the conductivity data, if any, and to the measured piezometric heads are generated. The details of the iSS implementation are explained next:

Consider that there are $N_{e}$ realizations in the ensemble, and that each realization is discretized into $N$ nodes.

1. Initialization step. We need to start from an ensemble of conductivity fields. This ensemble should be generated with the algorithm that is most adequate for the type of heterogeneity that describes the conductivity spatial variability. This ensemble can be made conditional to hard measurements of conductivity, and also to soft measurements such as those derived from geophysics. For the purpose of illustration, we choose a formation with channel-like features that introduce a high connectivity of the facies in the direction of flow. We choose this type of heterogeneity because it is well known that it is difficult to capture by multiGaussian-based approaches. In our synthetic case, the initial ensemble of realizations is generated in two steps. In the first step, an ensemble of binary facies realizations is constructed using direct simulation Mariethoz et al. (2010) - a very efficient implementation of sequential normal-equation simulation, first developed by Guardiano and Srivastava (1993a) and improved by Strébelle (2000). Then, each facies (channel/sand and non-channel/shale) is independently populated with log-conductivity values using sequential Gaussian simulation; the conductivities of each facies have very distinct mean values, ensuring that each realization has a clearly bimodal distribution, with the highest-value mode in the channel elements and the lowest-value mode in the non-channel elements. The specific parameters used for the generation of the initial ensemble of log-conductivities are described in the next section. At the end of this step there is an ensemble of hydraulic conductivity fields that will be denoted by $\mathbf{K}^{0}$, with $K_{i, j}^{0}$ being the conductivity for realization $i$ at node $j$. The superindex is used for the time coordinate and zero indicates that these are the initial conductivity estimates. For notation purposes, we will use $K_{i}$, to denote realization $i$ of the ensemble, and $K_{\cdot, j}$ to denote the set of $N_{e}$ conductivity values collected from all realizations at node $j$. During the initialization step it is also necessary to specify the initial piezometric heads $\mathbf{H}^{0}$, as well as boundary conditions and forcing terms necessary to solve the transient groundwater flow equation. 
2. Forecasting step. In this step, the simulated piezometric heads $\left(\mathbf{H}^{t+1}\right)$ are calculated for the $(t+1)^{\text {th }}$ time step based on the piezometric heads and the hydraulic conductivity estimates from the $t^{\text {th }}$ time step using a transient flow model:

$$
H_{i, \cdot}^{t+1}=\psi\left(H_{i, \cdot}^{t}, K_{i, \cdot}^{t}\right), \quad i=1, \ldots, N_{e} .
$$

The groundwater flow equation, represented by $\psi(\cdot)$ has to be solved independently for each realization of the ensemble.

3. Normal-score transformation step. A normal-score transformation will be applied to all the conductivity values of all the realizations:

$$
\widetilde{K_{i, j}^{t}}=G^{-1}\left(F_{j}\left(K_{i, j}^{t}\right)\right), \quad i=1, \ldots, N_{e} ; \quad j=1, \ldots, N .
$$

where $\widetilde{\mathbf{K}}^{\mathbf{t}}$ and $\mathbf{K}^{t}$ are the normal-score transformed hydraulic conductivity vector and the hydraulic conductivity vector estimates at time step $t$, respectively; $F(\cdot)$ is a vectorial normal-score transform function, with $N$ components, one for each location. The normal-score transform function is, generally, a non-parametric function that is built as described in Appendix A. After the normal-score transform of all the elements in all realizations, the transformed ensemble of normal-score conductivity realizations will follow a marginal Gaussian distribution with zero mean and unit variance.

4. Covariance calculation. As it will be explained later, for the updating step the normal-scored conductivity auto-covariance and the crosscovariances between normal-scored conductivity and piezometric heads will be needed. These covariances are non-stationary and need to be computed accounting for the locations of each variable. The procedure is described next. First consider the augmented variable vector

$$
\mathbf{S}=\left[\begin{array}{l}
\widetilde{\mathbf{K}} \\
\mathbf{H}
\end{array}\right]
$$

with $N_{e}$ realizations of $2 N$ variables, the covariance between any two variables $S_{., k}$ and $S_{., l}$ is given by

$C_{k, l}=\frac{1}{N_{e}} \sum_{m=1}^{N_{e}}\left(S_{m, k}-\left\langle S_{., k}\right\rangle\right)\left(S_{m, l}-\left\langle S_{., l}\right\rangle\right) \quad k=1, \ldots, 2 N ; l=1, \ldots, 2 N$

with

$$
\left\langle S_{., *}\right\rangle=\frac{1}{N_{e}} \sum_{m=1}^{N_{e}} S_{m, *}
$$


Since piezometric heads change in time, and hydraulic conductivities are also updated in time, the covariance is recalculated at each time step and, therefore, it is time dependent. The covariance matrix $\mathbf{C}$ contains $2 N \times 2 N$ elements; however, not all elements have to be computed, and the effective number of elements that must be computed is in the order of $N \log N$.

5. Sampling step. Piezometric heads are sampled at a few locations $N_{h}$ at time step $t+1$

6. Update step. In this step, a new ensemble of $\widetilde{\mathbf{K}}$ is generated conditioned to the hard conductivity measurements and to the just sampled piezometric heads. Sequential multivariate Gaussian simulation is used as outlined next; the reader interested in knowing all the implementation details of the algorithm is referred to (e.g., Gómez-Hernández and Journel, 1993; Deutsch and Journel, 1992; Delbari et al., 2009). The steps of the sequential simulation algorithm to generate realization $\widetilde{K_{i}}$. (the superindex $t+1$ is omitted por clarity):

(a) Assign the normal-score transformed values of the conditioning conductivity measurements to the closest nodes in the grid.

(b) Assign the observed piezometric heads to the closest nodes in the grid.

(c) Generate a random path through all $N$ grid nodes to be simulated.

(d) Visit a node along the random path. At the node location, search, within a predefined search neighborhood, all $\widetilde{K}$ values already in the grid, and all observed piezometric heads. Then, compute the conditional distribution function given the $\widetilde{K}$ and $H$ data found. Under the assumption of multivariate Gaussianity, this conditional distribution function is Gaussian and its mean and variance are given by the solution of a set of simple kriging equations (e.g., Deutsch and Journel, 1992; Goovaerts, 1997). Denoting the node for which the conditional distribution is to be computed by $j$, the row vector of $n$ conditioning data (normal-scored conductivities and piezometric heads) by $\mathbf{S}_{(n)}$, the covariance matrix between any two variables at the conditioning locations by $\mathbf{C}_{\alpha}$, and the covariance column vector between the conditioning locations and the location being estimated as $\mathbf{C}_{\beta}$, the conditional mean at $j$ is given by

$$
m_{\widetilde{K_{i, j}}}=\left\langle\widetilde{K_{i, j}}\right\rangle+\mathbf{C}_{\alpha}^{-1} \mathbf{C}_{\beta}\left(\mathbf{S}_{(n)}-\left\langle\mathbf{S}_{(n)}\right\rangle\right),
$$


where $\langle\cdot\rangle$ refers to the average value computed through the ensemble of realizations at a given location as in Eq. (5.5), and the conditional variance is given by

$$
\sigma_{\widetilde{K_{i, j}}}^{2}=C_{j, j}-\mathbf{C}_{\beta}^{T} \mathbf{C}_{\alpha}^{-1} \mathbf{C}_{\beta} .
$$

where $T$ denotes transpose. Note that the use of a search neighborhood limits the pairs of variables for which their covariance is needed while building $C_{\alpha}$ and $C_{\beta}$, it is for this reason that the number of covariance values that have to be precomputed at step 4 is in the order of $N \log N$.

(e) Draw a random number $\lambda$ from a standard Gaussian distribution with zero mean and unit variance, and generate $\widetilde{K_{i, j}}$ as

$$
\widetilde{K_{i, j}}=m_{\widetilde{K_{i, j}}}+\lambda \sqrt{\sigma_{\widetilde{K_{i, j}}}^{2}}
$$

(f) Assign $\widetilde{K_{i, j}}$ to node $j$ and return to step (d) to visit another node until all nodes in realization $i$ have been visited.

The update step is repeated for all realizations in the ensemble.

7. Back transformation step. Back transform the just generated normalscore transformed conductivities into conductivities using the inverse of the previously computed normal-score transform functions:

$$
K_{i, j}=F^{-1}\left(G\left(\widetilde{K_{i, j}}\right)\right), \quad i=1, \ldots, N_{e} ; \quad j=1, \ldots, N .
$$

8. Go back to the step 2 and repeat the process for as many time steps as there are observed piezometric heads.

The main difference between the iSS algorithm and the NS-EnKF algorithm is in the updating step. The updating step in the NS-EnKF (as in any other variant of the ensemble Kalman filter) is based on the premise that if there is a departure between forecasted piezometric heads and observed ones it is because there must be a departure between the conductivity estimates and their real values, and this departure can be computed by simple cokriging of the head departures. In the NS-EnKF, at each time step, there is a refinement of the conductivity fields according to the expression

$$
\widetilde{K_{i, j}^{t+1}}-\widetilde{K_{i, j}^{t}}=\mathbf{C}_{\alpha}^{-1} \mathbf{C}_{j, \beta}\left(\mathbf{S}_{(n)}-\mathbf{S}_{i,(n)}^{f}\right), \quad, i=1, \ldots, N_{e} ; j=1, \ldots, N
$$

where $\mathbf{S}_{(n)}$ is a vector with all the observed piezometric heads $\mathbf{S}_{i,(n)}^{f}$ is a column vector containing the forecasted piezometric heads at observation 
locations for realization $i, C_{\alpha}$ is a matrix with the covariances of forecasted heads at observation locations and $C_{j, \beta}$ is a vector with the cross-covariances between normal-scored conductivity at location $j$ and piezometric heads at observation locations.

\subsection{Synthetic Example}

A synthetic bimodal confined aquifer composed of $35 \%$ high permeability sand and $65 \%$ low permeability shale is constructed on a $50 \mathrm{~m}$ by $50 \mathrm{~m}$ square discretized into a grid of 50 by 50 by 1 cells. The thickness of the confined aquifer is assumed to be $5 \mathrm{~m}$. (The actual units are irrelevant for the purpose of the study, as long as consistency among them is kept.) The construction of the reference field is done in two steps. First, a two-facies field with 9 conditional data (Figure 5.1) is generated via the SNESIM code by Strebelle (2002) using the training image in (Strebelle, 2002) (see Figure 5.2). Second, this binary field is populated independently for each facies with log-conductivity values using a sequential Gaussian simulation code (GCOSIM3D) Gómez-Hernández and Journel (1993) with the parameters shown in Table 5.1. The resulting reference log-conductivity field and its histogram are shown in Figures 5.3 and 5.4. The two figures show that the distribution of log-conductivity is clearly non-Gaussian, the histogram has two modes (one for each facies) and the global mean and standard deviation are $-0.9 \ln (\mathrm{m} / \mathrm{d})$, and $2.9 \ln (\mathrm{m} / \mathrm{d})$, respectively.

A transient groundwater flow simulator MODFLOW (e.g., McDonald and Harbaugh, 1984; Harbaugh et al., 2000) is used to solve the transient groundwater equation. The model boundary is impermeable (see Figure 5.3). Figure 5.5 shows the locations of wells, including 25 observation wells, 2 injection wells and 3 pumping wells. Observation wells \# 6 and \# 7 will be used as calibration wells (post audit) and will not be used for conditioning. The injection rates of the two injection wells \# 1 and \# 2 are $16 \mathrm{~m}^{3} / \mathrm{d}$, and $15 \mathrm{~m}^{3} / \mathrm{d}$, respectively. The pumping rates of the three pumping wells \# 3 , \# 4, and \# 5 are $7.5 \mathrm{~m}^{3} / \mathrm{d}, 7.5 \mathrm{~m}^{3} / \mathrm{d}$ and $14.5 \mathrm{~m}^{3} / \mathrm{d}$, respectively. The initial head is set to $8 \mathrm{~m}$ throughout the study domain. Specific storage is

Table 5.1. Parameters of the random functions describing the spatial continuity of the sand and shale log-conductivities

\begin{tabular}{lccccccc}
\hline Facies & Proportion & $\begin{array}{c}\text { Mean } \\
{[\mathrm{ln}[\mathrm{m} / \mathrm{d}]]}\end{array}$ & $\begin{array}{c}\text { Std.dev } \\
{[\mathrm{ln}[\mathrm{m} / \mathrm{d}]]}\end{array}$ & $\begin{array}{c}\text { Variogram } \\
\text { type }\end{array}$ & $\begin{array}{c}\lambda_{x} \\
{[\mathrm{~m}]}\end{array}$ & $\begin{array}{c}\lambda_{y} \\
{[\mathrm{~m}]}\end{array}$ & sill \\
\hline Sand & 0.35 & 3.5 & 1.0 & exponential & 20 & 20 & 1 \\
Shale & 0.65 & -2.5 & 0.6 & exponential & 20 & 20 & 0.35 \\
\hline
\end{tabular}




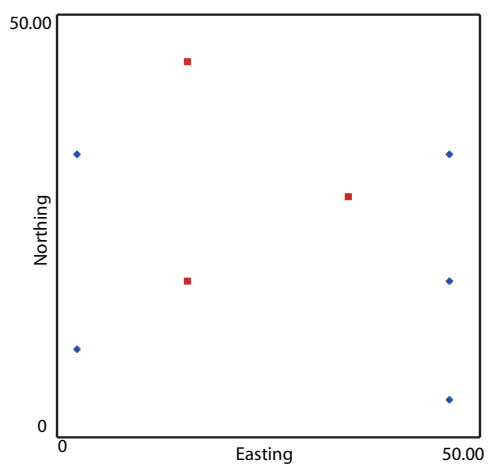

Figure 5.1. Location of the conditional data. The red nodes denote shale; the green nodes denote sand.

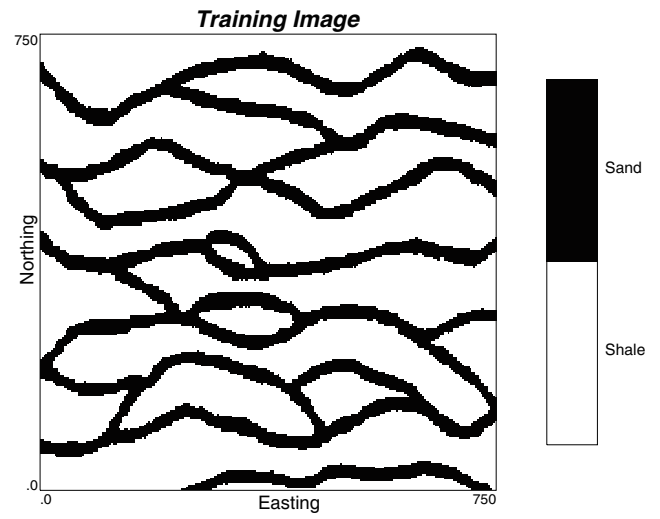

Figure 5.2. Training image used to generate the ensemble of binary facies realizations. 


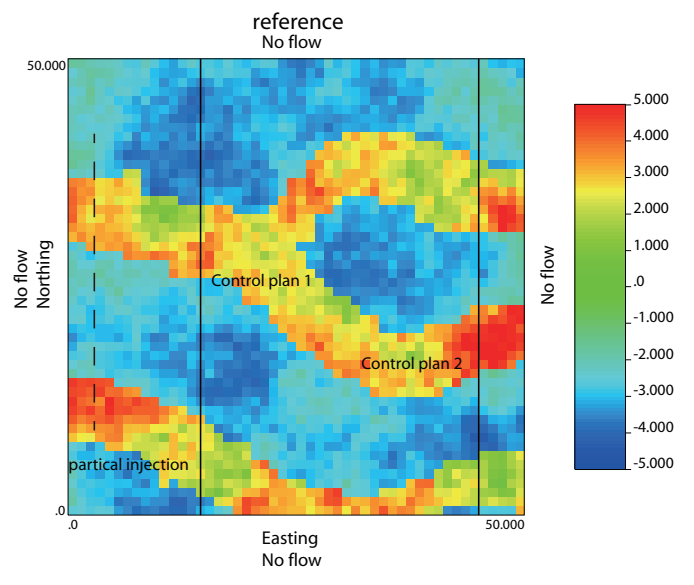

Figure 5.3. Reference field. It shows the boundary conditions; it also shows the source line (dashed) and the control planes at which breakthrough curves are computed (solid lines) in the transport experiment.

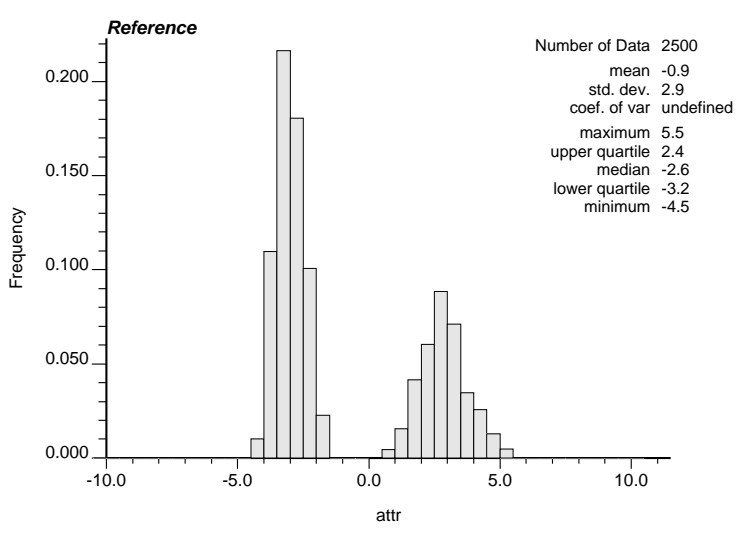

Figure 5.4. The histogram of the reference log-conductivity field 
set to $0.03 \mathrm{~m}^{-1}$. The total simulation time is 500 days, discretized into 100 time steps of increasing size following a geometric series with ratio 1.02; the length of the first time step is 1.60 days. The piezometric heads simulated in the reference field are sampled at the observation wells for each time step and used as input data for both algorithms.

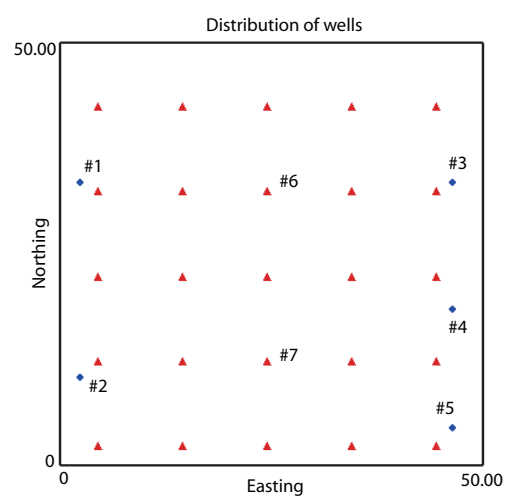

Figure 5.5. Location of wells. Red triangles denote observation wells; green squares denote injection (\#1, \#2) and pumping wells (\#3, \#4 and \#5). The observation wells \#6, \#7 are used as calibration wells.

As already mentioned, the performance of the iSS will be compared to that of the NS-EnKF. We will analyze two scenarios, scenario S0 with the results obtained applying the NS-EnKF, and scenario S1 with the results of the iSS. The data supplied to both algorithms is the same. Both algorithms use the same initial ensemble of 600 conductivity realizations. The initial ensemble of conductivity realizations is generated in a manner similar to which the reference field was generated: first an ensemble of facies realizations, conditional to the same 9 facies values as the reference, is generated using SNESIM; then, each facies is independently (and unconditionally) populated using GCOSIM3D with the same parameters in Table 5.1.

\subsection{Analysis}

Both the NS-EnKF and the iSS have been used to incorporate the observed piezometric heads during the first 50 time steps (135.4 days) for the generation of an ensemble of conductivity realizations. These realizations are analyzed by looking at several aspects:

1. Histogram of the ensemble. Figure 5.6a shows the log-conductivity histogram for the initial ensemble. Figure 5.6b,c display the log- 
conductivity histograms of the updated ensemble after the 50th time step for scenario S0, and scenario S1, respectively. We can see from Figure 5.6b,c that the histogram of log-conductivity with its bimodality is retained after 50 time steps in both scenarios.
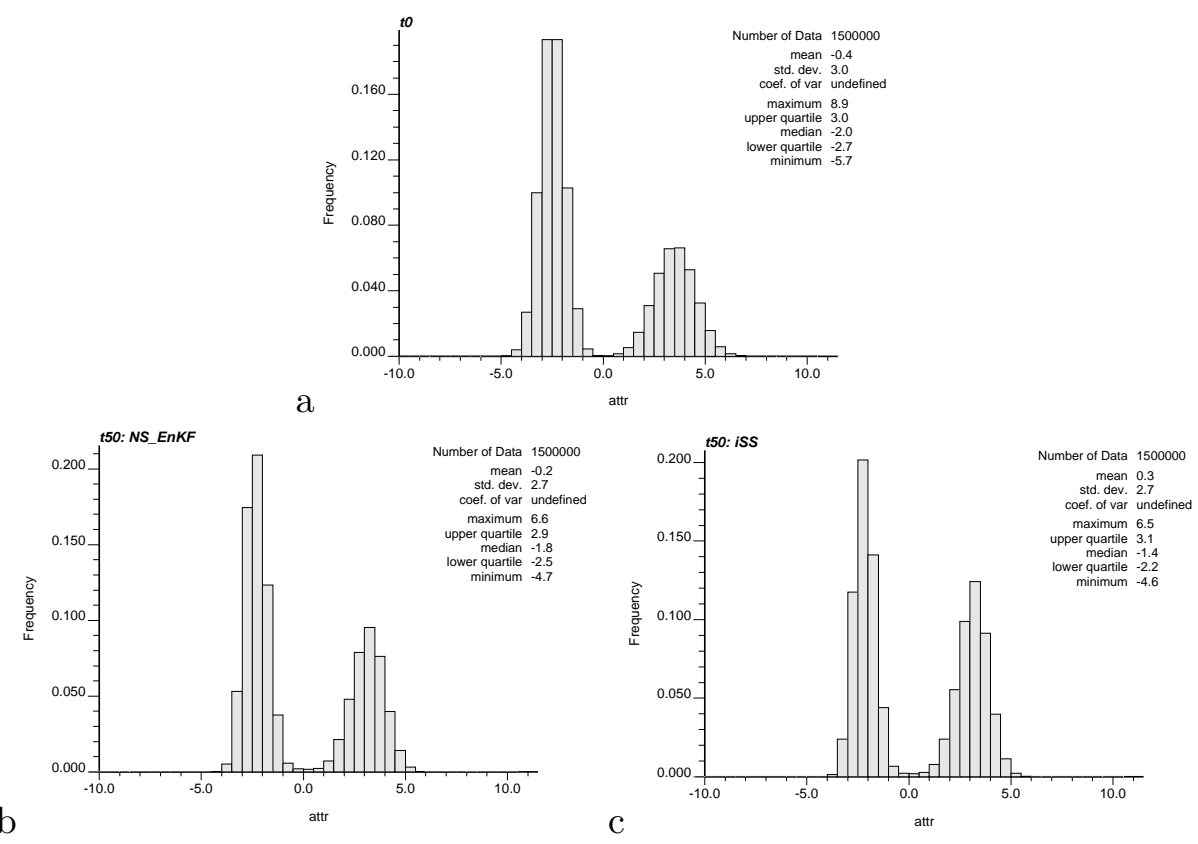

Figure 5.6. Log-conductivity histograms of the initial ensemble of realizations and of the updated ensemble of realizations after the 50th assimilation step of the two scenarios.

2. Ensemble mean and ensemble variance. Figure 5.7a,b shows the ensemble mean and variance for the initial ensemble. The only conditioning data used for the generation of the initial ensemble is the facies type at 9 locations. With this limited information it is not enough to control the spatial heterogeneity of each realization; therefore, the ensemble mean only shows some localized high and low values and the ensemble variance is quite high everywhere with only some small values around the facies conditioning locations. Figures 5.8 and 5.9 show the ensemble mean and ensemble variance, after 10 and 50 time steps. As time passes, and more piezometric heads are used to characterize the ensemble, the realizations of the ensemble are more alike, resulting in ensemble means that delineate the locations of the channels much better than in the initial set, and ensemble variances with zones of virtually no variance and small variances elsewhere. The areas with the highest ensemble variances are those areas with conductivity values which are not as sensitive to the piezometric heads at observation lo- 
cations as the rest of the aquifer; in a practical case, one could propose the sampling of conductivities at those locations. Both methods perform equally well, with the highest variance reductions for the longer times. The only significative difference is that the ensemble means obtained with the iSS algorithm appear to have a slightly larger short scale variability than the ensemble means obtained with the NS-EnKF.
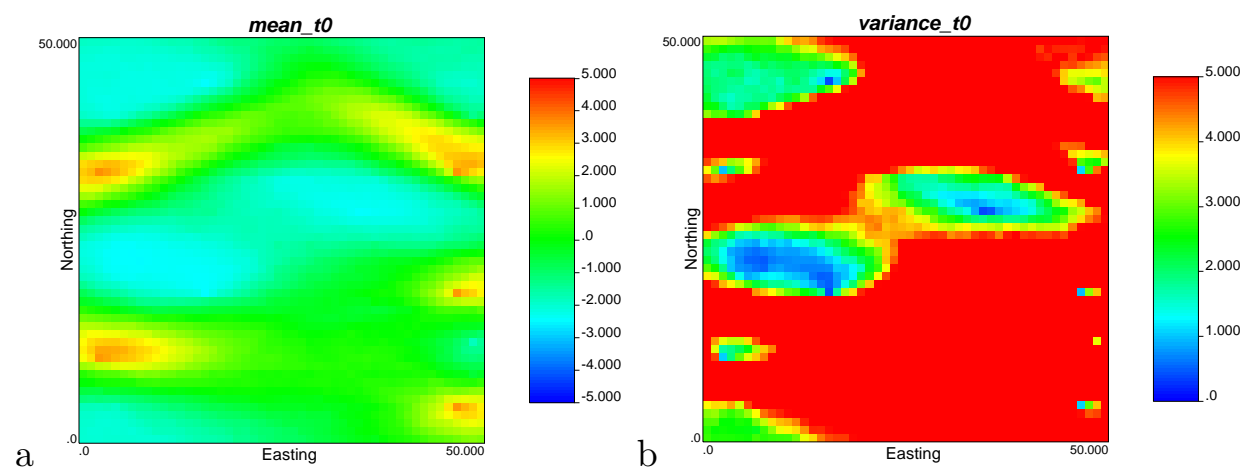

Figure 5.7. Ensemble mean and ensemble variance of $\ln K$ for the initial realizations.

3. Root mean square error (RMSE) and ensemble spreading (ES) of logconductivity. In synthetic examples like this one, we can calculate the deviation of the realizations from the "truth" since we have access to the underlying conductivity distribution from which the piezometric heads have been observed. The RMSE measures the accuracy of the algorithm in reproducing the reference field, and the ES measures the precision of the ensemble of realizations. The RMSE and ES are given by

$$
\begin{gathered}
R M S E=\sqrt{\frac{1}{N} \sum_{j=1}^{N}\left(\ln K_{j}^{r e f}-\left\langle\ln K_{j}\right\rangle\right)^{2}}, \\
E S=\sqrt{\frac{1}{N} \sum_{j=1}^{N} \sigma_{\ln K_{j}}^{2},}
\end{gathered}
$$

where $\ln K_{j}^{r e f}$ is the $\ln K$ value at node $j$ in the reference field, $\left\langle\ln K_{j}\right\rangle$ is the ensemble mean, and $\sigma_{\ln K_{j}}^{2}$ is the ensemble variance. As discussed by Chen and Zhang (2006) when the RMSE and the ES have a similar magnitude, the resulting ensemble variance provides a realistic measure of the uncertainty associated to the ensemble mean estimate.

Figure 5.10 shows the evolution in time of the RMSE, ES, and the ratio of RMSE to ES for both methods. We can see that the RMSE 
a
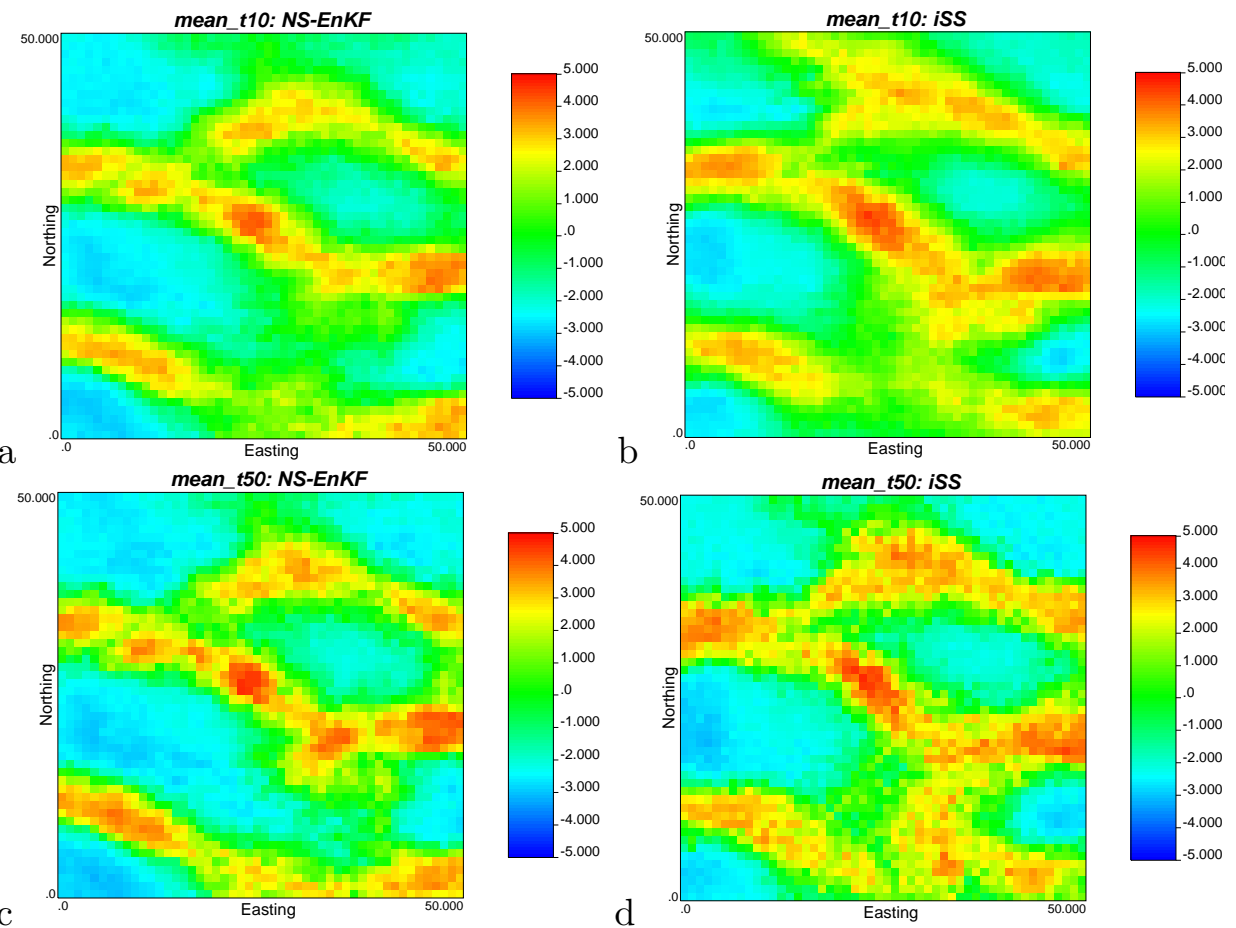

Figure 5.8. Ensemble mean of $\ln K$ after assimilating observation heads at the 10 th and 50th time steps for the two scenarios. 


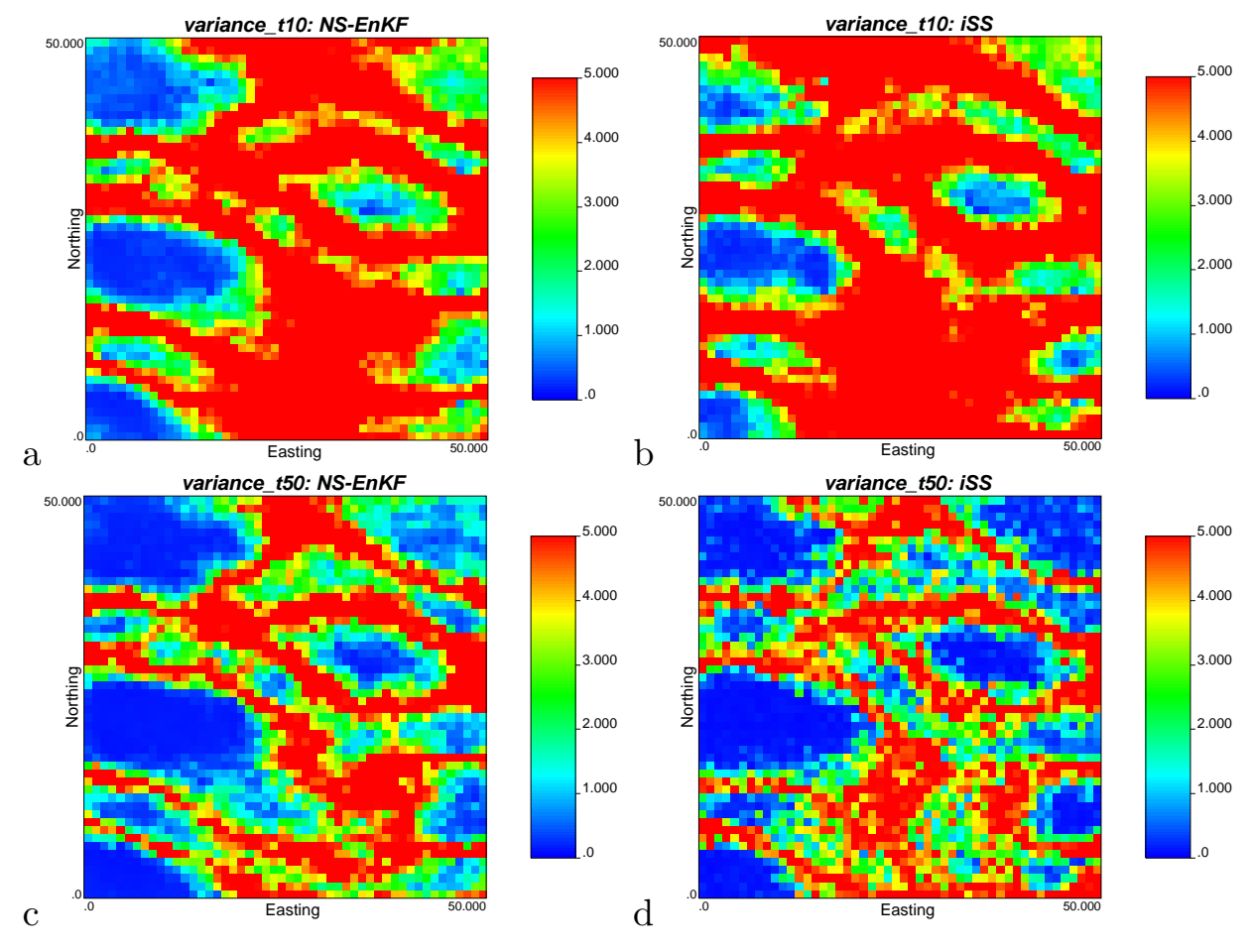

Figure 5.9. Ensemble variance of $\ln K$ after assimilating observation heads at the 10 th and 50th time steps for the two scenarios. 
corresponding to the NS-EnKF is smaller than that for the iSS, indicating that, on average, the realizations obtained with the NS-EnKF are closer to the reference than those obtained by iSS. The ES is similar in both cases. And the ratio RMSE/ES remains closer to 1 for the NS-EnKF than for the iSS, indicating a better characterization of the uncertainty with the former approach. Yet, these differences are small, and the absolute values of RMSE and ES sustain the conclusions derived from the visual analysis of the ensemble means and variances discussed before.

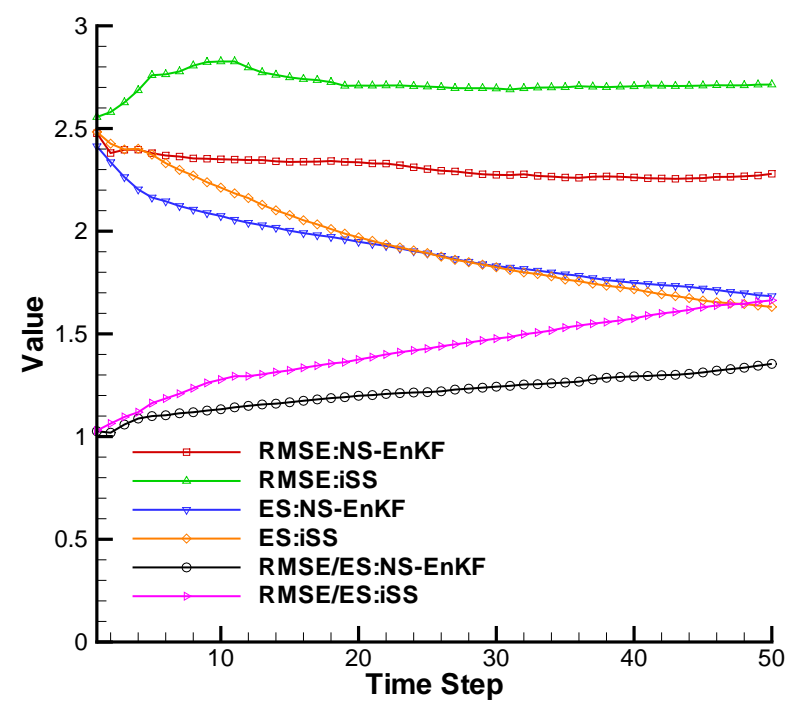

Figure 5.10. RMSE, ES, and RMSE/ES.

4. Reproduction of the piezometric heads at the two calibration wells. Figure 5.11 shows the evolution of the piezometric heads for the initial ensemble of conductivity realizations at the two calibration wells \#6 and \#7 - these wells were not used for conditioning. Since, no piezometric information was used to generate the initial conductivity ensembles, the spread among the individual responses of each realization is quite large. Figure 5.12 shows the evolution of the piezometric heads in the updated ensembles for both approaches. The vertical dashed line indicates the end of the use of the observed piezometric heads as conditioning data. Comparing Figures 5.11 and 5.12, the effect of conditioning on piezometric heads is patent, with a significant reduction of the spread of the piezometric head curves in all realizations about the reference one for both approaches. 
a

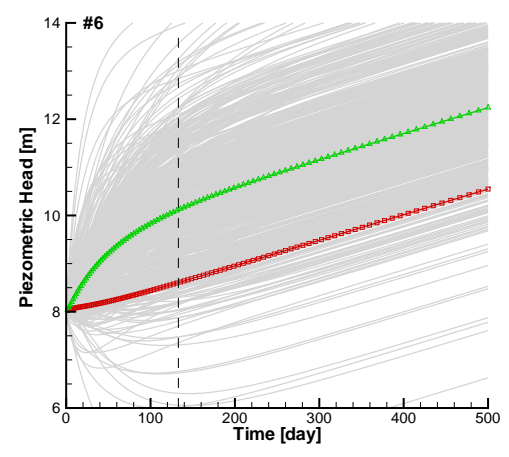

$\mathrm{b}$

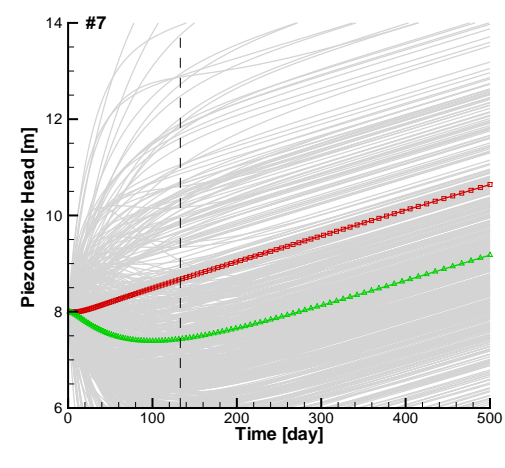

Figure 5.11. Evolution in time of the piezometric head at the two calibration wells for the initial ensemble of log-conductivity realizations. The red square line corresponds to the piezometric head in the reference, the gray lines correspond to the realizations and the vertical dashed lines marks the end of the conditioning period, the green delta line corresponds to the average of the gray lines.

5. Solute breakthrough curves (BTCs). A transport prediction experiment of an inert solute is carried out to further evaluate the goodness of the characterization of conductivity by the updated conductivity realizations. For this purpose, 10000 conservative particles are released along a vertical line at $x=2.5 \mathrm{~m}$ and the arrival times are recorded at two control planes, located at $x=15 \mathrm{~m}$ and $x=45 \mathrm{~m}$ (see Fig. 5.3). The random walk particle tracking program RW3D (FernàndezGarcia et al., 2005) is used to solved the transport equation. Porosity is assumed constant and equal to 0.3. Figure 5.13 Shows the BTCs at the two control planes for the initial ensemble. Figure 5.14 shows the BTCs at the two control planes using the conductivity fields updated after 50 times steps. We can see that the uncertainty about the BTC predictions is significantly reduced after conditioning to the piezometric head data. Comparing the Figure 5.14b,d with Figure 5.14a,c, we can find that the iSS performs a little better than the NS-EnKF, since the spread of the BTC predictions is smaller, and the median BTCs is closer to the reference BTCs. 
a
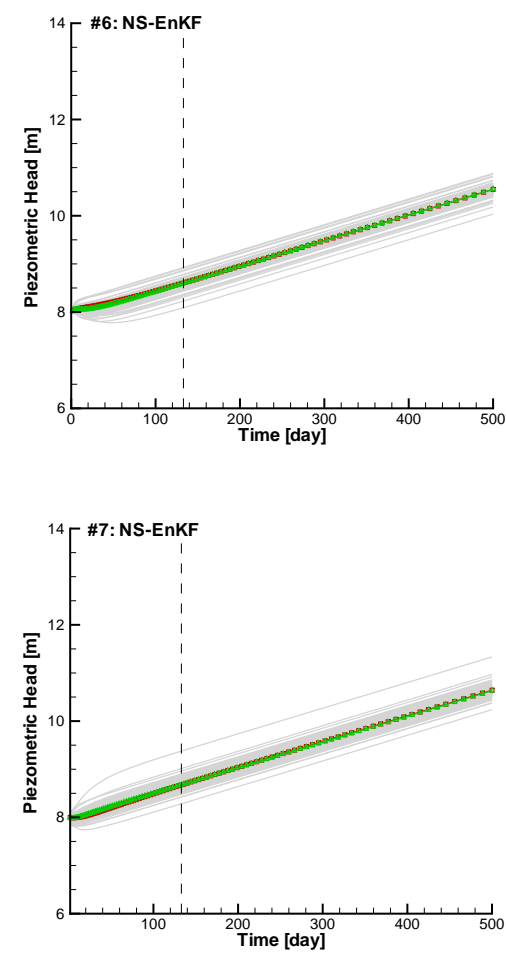

$\mathrm{b}$

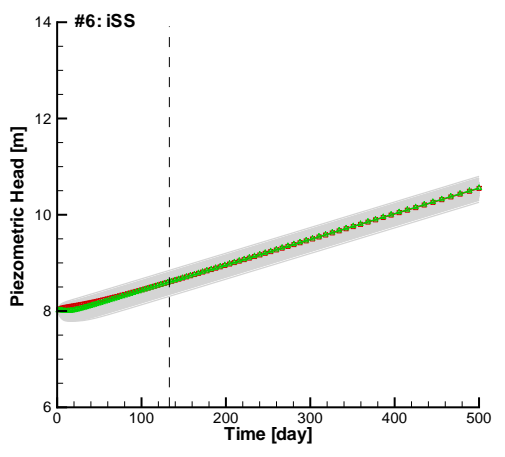

d

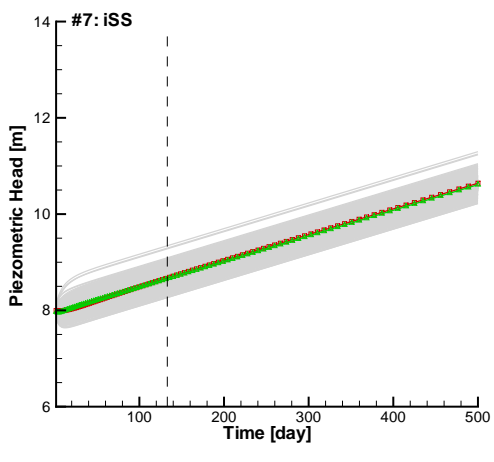

Figure 5.12. Evolution in time of the piezometric head at the two calibration wells after the 50th time step. The red square line corresponds to the piezometric head in the reference, the gray lines correspond to the realizations and the vertical dashed lines marks the end of the conditioning period, the green delta line corresponds to the average of the gray lines. 

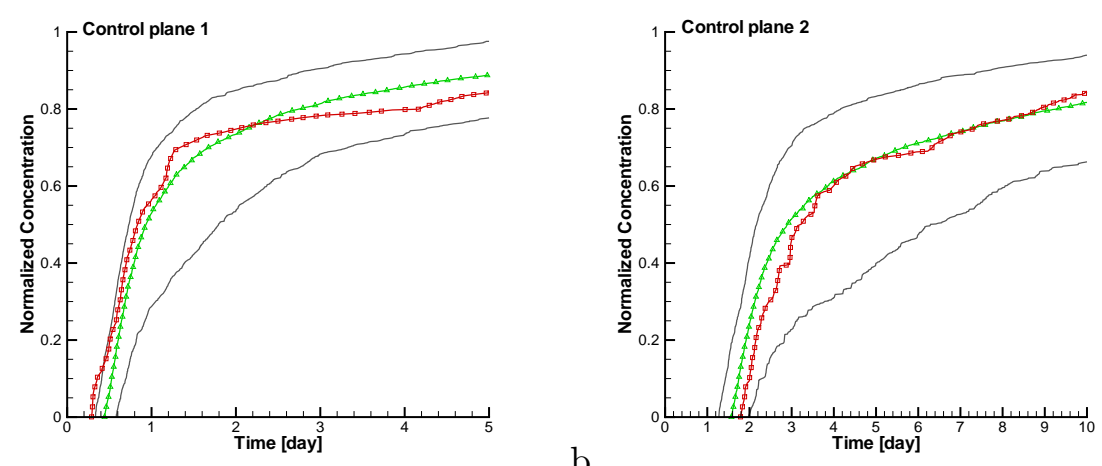

a

$\mathrm{b}$

Figure 5.13. Breakthrough curves (BTC) at the two control planes for the initial $\ln K$ realizations. The red square line corresponds to the BTCs in the reference. The black lines correspond to the 5 and 95 percentiles of all realization BTCs, and the green delta line corresponds to the median.

a
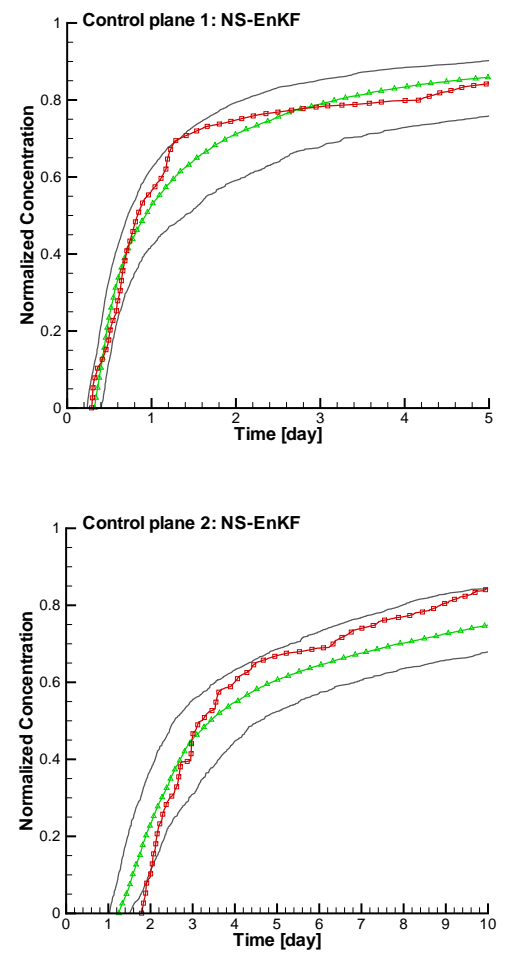

$\mathrm{b}$
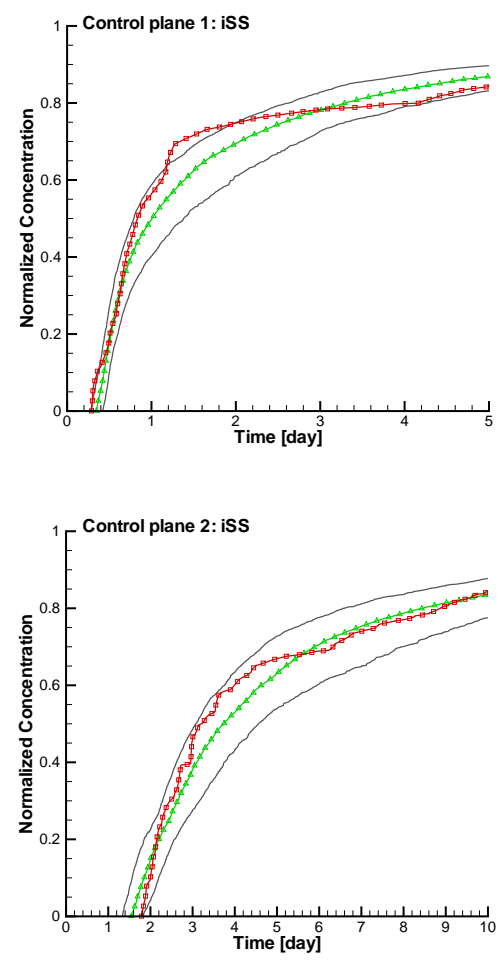

Figure 5.14. Breakthrough curves (BTC) at the two control planes for the $\ln K$ realizations updated after the 50th time step. The red square line corresponds to BTCs in the reference. The black lines correspond to the 5 and 95 percentiles of all realization BTCs, and the green delta line corresponds to the median. 


\subsection{Discussion}

From the above analysis we can conclude that the quality of the ensemble of realizations generated by iSS is comparable to that of the ensemble generated by NS-EnKF. The main difference between the two approaches is that iSS is a stochastic simulation approach, whereas the NS-EnKF works by progressively refining an initial ensemble of realizations on the basis of the discrepancy between forecasted and predicted piezometric heads. In the iSS, at each time step, a new ensemble of realizations is generated using as conditioning data the last set of observed piezometric heads; the ensemble of realizations keeps improving as time progresses, through the updating of the experimental ensemble non-stationary covariances. Furthermore, since iSS is based in the sequential simulation algorithm, which uses a search neighborhood to decide the information to use when computing the cumulative distribution function at a given location, it avoids the use of spurious correlations for long distances, since conditioning data which are far from the point being simulated are never used, solving, in this way, a common problem of the ensemble Kalman filter implementations. There is nothing that prevents the use of the iSS algorithm to condition not only on the observed piezometric heads at time $t+1$, but simultaneously on piezometric heads measured at time $t$, and at other previous times; including these additional past data would imply only computing the cross-covariances between normal-scored conductivities and the forecasted piezometric heads at the earlier steps, and could improve the final characterization of conductivities. The power of iSS, as compared with other stochastic simulation techniques, is based on the same principle that has made the ensemble Kalman filter so successful, the use of non-stationary covariances experimentally computed from a set of ensemble realizations of conductivity and the resulting ensemble of piezometric heads obtained after running a numerical flow model on the conductivity realizations. Notice that iSS, being an inverse simulation technique, is neither an optimization algorithm nor a filter, it is a stochastic simulation technique. The algorithm is still in its first stages, but we are confident that it could be used for inverse stochastic simulation under a wide variety of scenarios, not limited to the field of hydrogeology.

\subsection{Summary and conclusions}

A new inverse stochastic simulation method, the inverse sequential simulation (iSS), has been proposed for the purpose of generating a set of hydraulic conductivity realizations that, when used to model groundwater flow, can reproduce observed piezometric heads. The method is based on the sequential simulation paradigm making use of the non-stationary covariance experimentally inferred form an ensemble of conductivity fields and the cor- 
responding piezometric head responses, in a manner similar as how it is done in ensemble Kalman filtering. We have benchmarked the technique against the normal-score ensemble Kalman filter (NS-EnKF), possibly the current algorithm that best performs for inverse modeling using transient piezometric heads. The iSS compares very well with the NS-EnKF in all aspects analyzed (histogram reproduction, ensemble mean and variance, reproduction of observed piezometric heads, and reproduction of breakthrough curves), and it can be considered an alternative approach for inverse stochastic simulation. The new algorithm has room for expansion and has the potential of application in other fields. 


\title{
Inverse Sequential Simulation: Performance and Implementation Details
}

\begin{abstract}
For good groundwater flow and solute transport numerical modeling, it is important to characterize the formation properties. In this paper, we describe a new approach for stochastic inverse modeling called inverse sequential simulation (iSS) capable of characterizing conductivity fields with heterogeneity patterns difficult to capture by standard multiGaussian-based inverse approaches. The method is based on the multivariate sequential simulation principle, in which the local conditional probability distributions are computed, by simple co-kriging, using the expected values, covariances and cross-covariances derived from an ensemble of conductivity and piezometric head fields. The piezometric head fields are the solution of the groundwater flow equation for each member of the ensemble of conductivities with given initial and boundary conditions, and recharge and pumping schedules. Expected values, covariances and cross-covariances are updated in time as new piezometric head data are acquired. The details of its implementation are described and the sensitivity of the approach to different input parameters is shown.
\end{abstract}




\subsection{Introduction}

Characterization of geological properties plays an important role in making reliable groundwater models. Inverse modeling is a powerful tool to improve such a characterization by incorporating the information about the geological properties born by the state variables. The reader is referred to the work by Zhou et al. (2013) for a recent review on inverse methods in hydrogeology.

There has been a lot of activity in this field in the last decades, and many approaches have been developed. Just to name a few, there are the gradual deformation method, the sequential self-calibration, variants of the Markov chain Monte Carlo method, the representer method, the pilot points method, particle filters, ... (e.g., Capilla and Llopis-Albert, 2009; Hu, 2000; Gómez-Hernánez et al., 1997; Fu and Jaime Gómez-Hernández, 2009; Oliver et al., 1997; Alcolea et al., 2006; Wen et al., 2002; RamaRao et al., 1995; Franssen et al., 2003; Gordon et al., 1993; Losa et al., 2003; Van Leeuwen, 2009).

Besides the above mentioned methods, the ensemble Kalman filter (EnKF) Evensen (2003) is the method that has attracted most attention recently. Although not an inverse method by conception, the inclusion of the parameters governing the state equation as part of an extended state vector, has turned the ensemble Kalman filter in the most favored inverse method nowadays. The EnKF is well known for its flexibility to be applied to virtually any inverse problem, and for its efficiency in producing realizations of the geological parameters that are consistent with the observed state variable data.

But the EnKF has been shown to fail when applied to scenarios in which the underlying geological properties exhibit patterns of spatial variability that are difficult, if not impossible, to be reproduced by realizations drawn from a multiGaussian probability distribution (e.g., Sun et al., 2009; Simon and Bertino, 2009). The main reason being that the EnKF relies only on twopoint statistics, that is, covariances and cross-covariances; and therefore, it is optimal only for multiGaussian variates and linear state transfer functions Aanonsen et al. (2009). For this reason, the main focus on inverse modeling, at least in the fields of hydrogeology and petroleum engineering, is how to capture those non-multiGaussian patterns in inverse modeling.

The development of the sequential normal-equation simulation (Guardiano and Srivastava, 1993a; Strébelle, 2000) has allowed to use statistics higher than order two in the construction of realizations, and thus, the inclusion of the spatial features that cannot be characterized simply by a covariance function on the final models of the geological parameters. The algorithms that are capable to account for statistics higher-than-order-two are broadly termed as multiple point statistics (MPS). They rely on the existence of a training image exhibiting the types of patterns to be present in the final models, from which to infer the higher-order statistics. Some available algo- 
rithms and codes are SNESIM (Strebelle, 2002), FILTERSIM (Zhang et al., 2006), SIMPAT (Arpat and Caers, 2007), DS (Mariethoz et al., 2010), EnPAT (Li et al., 2013), and others (e.g., Zhou et al., 2012; Chugunova and Hu, 2008; Mirowski et al., 2009; Boucher, 2009; Honarkhah and Caers, 2010).

There have been some attempts to combine MPS and inverse modeling: $\mathrm{Hu}$ et al. (2012a) used the realization of uncorrelated random numbers needed for the drawing of the conductivity value from the local distribution function on sequential normal-equation simulation as the state variables to be updated during the analysis step if the EnKF, with moderate success; Zhou et al. (2012); Li et al. (2013) developed a new MPS algorithm (termed EnPAT) which blends direct simulation (Mariethoz et al., 2010) and the EnKF to generate inverse conditional realizations of conductivity in channelized bimodal aquifers; EnPAT works well, but it is still very CPU-time consuming. Some authors are against the use of MPS arguing that they are too dependent on the choice of the parameters controlling the algorithm (Mustapha and Dimitrakopoulos, 2010).

Other approaches to address the issue of non-multiGaussianity include the works by Sun et al. (2009), who combined the EnKF with a Gaussian mixture model, or by Liu and Oliver (2005); Gu and Oliver (2007); Franssen and Kinzelbach (2008) who use an iterative EnKF; plus a set of works who combine the normal-score transform (sometimes referred as anamorphosis) and the EnKF (e.g., Simon and Bertino, 2009, 2012; Bertino et al., 2003a,b; Bocquet et al., 2010; Béal et al., 2010; Schöniger et al., 2012; Zhou et al., 2011; Li et al., 2012b).

In this paper we describe a new approach for inverse stochastic modeling, applicable for non-multiGaussian fields, which is based on two-point statistics and on the normal-score transform, termed inverse sequential simulation. Inverse sequential simulation is inspired on the standard sequential simulation algorithm and the Monte Carlo concept of the EnKF. The paper describes, in detail, the algorithm and its implementation, and then performs a sensitivity analysis of the key parameters controlling the algorithm.

\subsection{Methodology}

The key idea of inverse sequential simulation (iSS) is to use multivariate multi-Gaussian sequential simulation (Gómez-Hernández and Journel, 1993) to generate realizations of normal scores of conductivity, conditioned on conductivity and piezometric head data. The main difference with standard sequential simulation is that the method does not use an analytical, stationary model for the auto- and cross-covariances, but rather, as in the ensemble Kalman filter, the experimental non-stationary auto- and crosscovariances are derived from an ensemble of conductivity realizations and 
their associated piezometric heads (obtained by solving a groundwater flow model).

Before describing the whole algorithm, recall the main steps in any sequential simulation algorithm are:

1. Define a random path to visit all nodes of the grid on which the realization will be generated.

2. Visit the random path sequentially.

(a) At each node, collect the conditioning data (for all variables) within a user-defined search neighborhood centered at the point to simulate (the size and orientation of the search neighborhood, the number of data to keep within it, and how the retained data should be distributed between the different variables are parameters that must be specified by the user).

(b) Compute the local conditional distribution function. If we adopt a multivariate multiGaussian random function model, the local conditional distribution is a Gaussian distribution with mean and variance given by the simple co-kriging estimate and the simple co-kriging variance.

(c) Draw, randomly, a value from the local conditional distribution function.

(d) Include the value in the set of conditioning data for the simulation of the next nodes and go to next node.

Our proposal is to use this algorithm to generate conductivity fields conditioned to piezometric heads. For this purpose we need the autocovariances of both conductivity and head, and their cross-covariance. These covariances, particularly the ones involving the piezometric heads, but also the conductivity auto-covariance when there are conditioning conductivity data, are clearly non-stationary. Some authors have developed analytical expressions relating these covariances by approximating the solution of the groundwater flow equation (e.g., Fiori et al., 1998). We propose to use experimentally-derived covariances obtained from an ensemble of realizations, much like it is done in the ensemble Kalman filter.

At any time $t$, we could derive all necessary covariances experimentally as follows:

1. Generate an ensemble of $N_{e}$ realizations of conductivity. Each realization contains $N$ nodes. $K_{i}(j)$ refers to the conductivity value at realization $i$ and node $j$.

2. Given initial and boundary conditions, sources and sinks, solve the groundwater flow equation for each realization until time $t$ and obtain an ensemble of piezometric heads. 
3. The cross-covariance between conductivity $K$ at location $j$ and piezometric head $H$ at location $l$ is given by

$$
C_{K, H}(j, l)=\frac{1}{N_{e}} \sum_{i=1}^{N_{e}}\left(K_{i}(j)-\bar{K}(j)\right)\left(H_{i}(l)-\bar{H}(l)\right)
$$

where the overbar indicates ensemble average, i.e.,

$$
\bar{K}(j))=\frac{1}{N_{e}} \sum_{i=1}^{N_{e}} K_{i}(j)
$$

In addition, since we are planning to work with multiGaussian sequential simulation, it is more convenient to work with the normal-score transform of the variable of interest, in our case conductivity. Therefore, the sequential simulation algorithm is performed on a new variable $\widetilde{K}$ which is obtained by the normal-score transform of $K$ according to the following expression:

$$
\widetilde{K}_{i}(j)=G^{-1}\left(F_{j}\left(K_{i}(j)\right)\right)
$$

where $F_{j}\left(K_{i}(j)\right.$ is the local cumulative distribution at node $j$ computed (numerically) from the $N_{e}$ conductivity values of all realizations at node $j$, and $G(\cdot)$ is the standard Gaussian cumulative distribution function.

Once the normal-scores are generated, they are transformed back to conductivity space for the solution of the groundwater flow equation using the inverse expression:

$$
K_{i}(j)=F_{j}^{-1}\left(G\left(\widetilde{K}_{i}(j)\right)\right) .
$$

We expect that by using a good approximation of the non-stationary cross-covariance between conductivity and piezometric head, the resulting conditional conductivity fields will be coherent with the piezometric heads, in the sense that the solution of the groundwater flow equation on the conditional fields will reproduce the observed piezometric heads. However, we found that this reproduction does not happen, at least in first instance. We found that, for the algorithm to work, we have to apply an iterative approach by introducing piezometric heads measured at different time steps and recomputing the covariances after each time step.

Under these considerations, the inverse sequential simulation algorithm consists of the following steps:

1. Define the parameters controlling the sequential simulation: search neighborhood, maximum number of conditioning points to retain within the search neighborhood, number of realizations to generate.

2. Define the parameters controlling the groundwater flow simulation: initial conditions, boundary conditions, sources and sinks. 
3. Generate an initial ensemble of conductivity fields conditioned to measured conductivity data. This ensemble contains $N_{e}$ realizations discretized into $N$ nodes. Any stochastic simulation technique can be used to initialize this ensemble, whether multiGaussian-based or not. (In the example below we will use a hybrid of multiple-point simulation and Gaussian sequential simulation to generate realizations with channel-like patterns of sand and shale.)

4. Begin iteration in time.

(a) Solve, for each conductivity realization, the transient groundwater flow equation from the previous time until time $t$. The result is an ensemble of piezometric heads $H$.

(b) Normal-score transform the conductivity realizations.

(c) Compute the auto- and cross-covariances of $\widetilde{K}$ and $H(t)$.

(d) Sample piezometric heads at observation locations. These values will be used as conditioning data.

(e) Generate a new ensemble of normal-score conductivity fields by multivariate multiGaussian sequential simulation using both conductivity and piezometric head as conditioning data.

(f) Backtransform the normal-score conductivities into conductivities and move on to the next time step.

After each time iteration, a new set of conductivity fields results, which is used to forecast the piezometric heads to the next time. It is important to notice that after each iteration, the new set of conductivity fields will have a different statistical structure than the previous one, that is, different local cumulative distributions, different ensemble mean, different ensemble variance, different covariance, and different cross-covariance with the piezometric heads as a result of the conditioning effect in the sequential simulation step. For those readers familiar with the EnKF, there is a fundamental difference in that iSS builds from scratch the new ensemble of conductivities at each time step, whereas in the EnKF, each realization of the ensemble is updated, at each time step, by adding to it a smooth perturbation.

\subsection{Synthetic Example}

We wish to test the performance of the iSS algorithm in an aquifer with spatial heterogeneity that is not suited for modeling with a multiGaussian random function. The marginal histogram of logconductivities must be bimodal, and conductivities must show strong connectivity for the high values. It is well known that these two aspects are difficult to handle by a multiGaussian random function model (Gómez-Hernández and Wen, 1998). 
Table 6.1. Parameters of the random functions describing the spatial continuity of the sand and shale log-conductivities

\begin{tabular}{lccccccc}
\hline Facies & Proportion & $\begin{array}{c}\text { Mean } \\
{[\ln [\mathrm{m} / \mathrm{d}]]}\end{array}$ & $\begin{array}{c}\text { Std.dev } \\
{[\ln [\mathrm{m} / \mathrm{d}]]}\end{array}$ & $\begin{array}{c}\text { Variogram } \\
\text { type }\end{array}$ & $\begin{array}{c}\lambda_{x} \\
{[\mathrm{~m}]}\end{array}$ & $\begin{array}{c}\lambda_{y} \\
{[\mathrm{~m}]}\end{array}$ & sill \\
\hline Sand & 0.35 & 3.5 & 1.0 & exponential & 20 & 20 & 1 \\
Shale & 0.65 & -2.5 & 0.6 & exponential & 20 & 20 & 0.35 \\
\hline
\end{tabular}

We use as reference a synthetic confined aquifer composed of sand and shale. The aquifer occupies an area of $50 \mathrm{~m}$ by $50 \mathrm{~m}$ by $5 \mathrm{~m}$ and it is discretized into a grid of 50 by 50 by 1 cells. The proportion of sand to shale is 0.35 to 0.65 . The facies distribution should be consistent with the training image of Figure 6.1 taken from Strebelle (2002). (Note that the training image extends over a much larger area than the aquifer.)

The synthetic aquifer is built in two steps. On the first step a binary sand/shale realization is generated using the training image in Figure 6.1 and eight conditioning points distributed as shown in Figure 6.2. The code used in this first step is SNESIM Strebelle (2002). In the second step, the facies are populated with log-conductivity values generated, independently, by sequential Gaussian simulation. The code used in this second step is GCOSIM3D Gómez-Hernández and Journel (1993). The parameters used for the generation within each facies are listed in Table 6.1. The resulting field is shown in Figure 6.3, where the channelized structure is clearly seen. The histogram of log-conductivities in the reference aquifer is shown in Figure 6.4 , it displays two modes at $3.5 \ln (\mathrm{m} / \mathrm{d})$ and $-2.5 \ln (\mathrm{m} / \mathrm{d})$, its global mean is $-0.9 \ln (\mathrm{m} / \mathrm{d})$ and its global standard deviation is $2.9 \ln (\mathrm{m} / \mathrm{d})$.

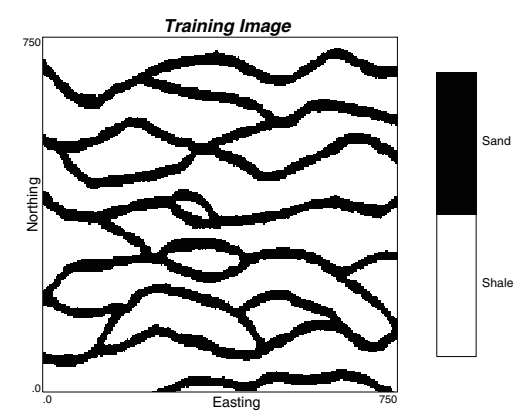

Figure 6.1. Training image used to generate the ensemble of binary facies realizations

Groundwater flow is solved in the aquifer assuming no flow boundary conditions, an initial head set at $8 \mathrm{~m}$ everywhere, two injection wells (coin- 


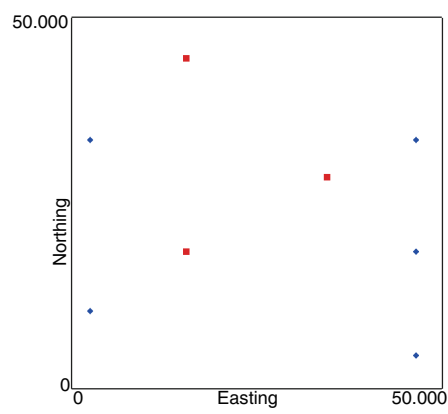

Figure 6.2. Location of the facies data, red nodes for shale and green nodes for sand

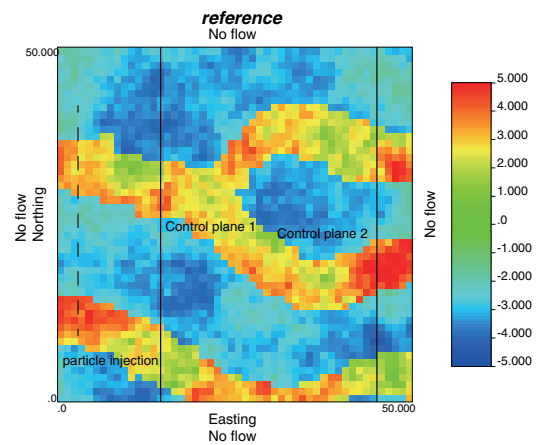

Figure 6.3. Reference field

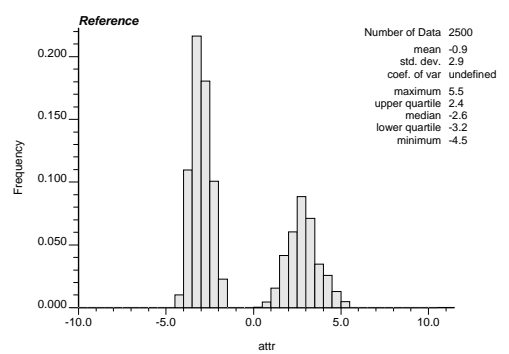

Figure 6.4. Histogram of the reference field 
Table 6.2. Definition of scenarios

\begin{tabular}{lcccccc}
\hline Scenario & S1 & S2 & S3 & S4 & S5 & S6 \\
\hline Number of observation piezometers & 25 & 25 & 25 & 25 & 16 & 25 \\
Number of maximum search nodes & 24 & 24 & 24 & 8 & 24 & 24 \\
Size of the ensemble of realizations & 600 & 600 & 600 & 600 & 600 & 100 \\
Number of conditional facies data & 8 & 0 & 16 & 8 & 8 & 8 \\
\hline
\end{tabular}

ciding with the two facies data in the sand facies along the west side of the aquifer) injecting $16 \mathrm{~m}^{3} / d$ (top one) and $15 \mathrm{~m}^{3} / d$ (bottom one), and three pumping wells (coinciding with the three facies data in the sand facies along the east side of the aquifer) pumping $7.5 \mathrm{~m}^{3} / \mathrm{d}, 7.5 \mathrm{~m}^{3} / \mathrm{d}$ (top two ones) and $14.5 \mathrm{~m}^{3} / \mathrm{d}$ (bottom one). The aquifer specific storage is homogeneous and equal to $0.03 \mathrm{~m}^{-1}$.

The transient groundwater flow simulator MODFLOW (e.g., McDonald and Harbaugh, 1984; Harbaugh et al., 2000) is used to solve the transient groundwater flow equation for a period of 500 days. The time span is discretized into 100 time steps whose length follows a geometric series of ratio 1.02. (The first time step is 1.6 days.)

The piezometric heads are sampled at each time step at the locations indicated below, these values will be used for conditioning during the first 50 time steps (135.4 days) and then used for validation afterwards.

We have designed six scenarios to make a sensitivity analysis to several parameters. These scenarios are described in Table 6.2. The scenarios differ between them in the number of conditioning facies data, in the number of realizations in the ensemble, in the maximum number of conditioning data retained in the sequential simulation step for the computation of the local conditional distribution function, and in the number of piezometers at which piezometric head is observed.

The procedure to generate the initial set of realizations for all six scenarios is the same one used to generate the reference field. They only differ in the number of facies conditional data. Scenario S2 has no conditioning data, scenarios S1, S4, S5 and S6 use the same 8 conditioning data used to generate the reference field (see Figure 6.2), and scenario S3 uses 8 additional conditioning data sampled from the reference field. The location and values of these 16 data can be seen in Figure 6.5.

All scenarios use 25 piezometers for sampling the piezometric head, except for scenario S5 that uses only 16. The location of these piezometers is shown in Figure 6.6. This figure also shows the locations of the two piezometers used to illustrate the performance of the method (labeled \#6 and \#7). 


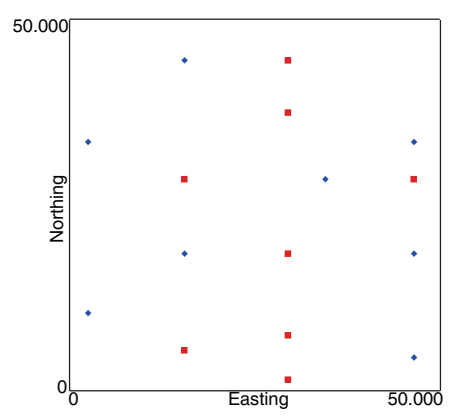

Figure 6.5. Location of the facies data for scenario S3, the one with a larger number of conditioning points; red nodes for shale and green nodes for sand
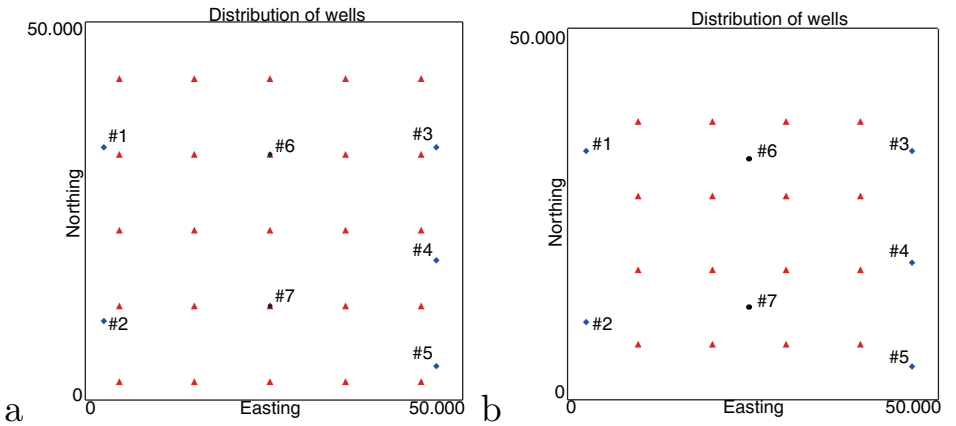

Figure 6.6. (a) Distribution of piezometers of scenarios S1, S2, S3, S4 and S6; (b) Distribution of piezometers for scenario S5. For reference, the injection (\#1, \#2) and pumping wells (\#3, \#4 and \#5) are also shown. The piezometric head evolution at piezometers \#6, \#7 is used in the analysis. 


\subsection{Analysis}

We have generated six sets of conductivity realizations according to the parameters in Table 6.2, next we are going to analyze up to which point they are truly conditional on the piezometric heads in the sense that observations are matched by groundwater flow predictions. Then, we will analyze the different sets against the reference field to check how close these realizations fluctuate about the reference field and how large these fluctuations are. And finally, we will perform a postaudit analysis to check how these sets of realizations will perform under completely different conditions, more precisely, how these realizations will reproduce solute transport as observed in the reference field.

\subsubsection{Conditioning to heads}

Figure 6.7 shows the piezometric heads evolution at piezometers \#6 and \#7 of Figure 6.6 on the initial sets of realizations, that is, those realizations that have been generated only conditional on facies data, since no piezometric head information has been used yet. As expected, the scatter of the piezometric heads is very wide. Figure 6.8 shows the same results after having conditioned to piezometric heads in the first 50 time steps (up to the vertical dashed line). Recall that this means that for each scenario 50 sets of realizations have been generated, one for each time step based on the auto- and cross-covariances computed on the set of realizations from the previous time step. The results are quite convincing with regard to the objective of iSS: the generation of realizations that match the observed piezometric heads. Comparing with Figure 6.7, the reduction of the spread is remarkable. Comparing among the graphs in Figure 6.8 we can appreciate that the conditioning is not equally good for all scenarios or both piezometers. It is clear that the best results are obtained for the set of realizations with the largest ensemble size and the largest values for all parameters (S3). Using the results for scenario S1 as benchmark we can also conclude that reducing the number of conditioning nodes to be retained with the search neighborhood during the sequential simulation step also reduces the quality of the conditioning to head observations. Finally, we can comment that apparently, reducing the size of the ensemble improves the reproduction of piezometric heads but, as we will discuss afterwards, reducing the number of realizations has as a consequence a drastic reduction of the experimental covariances producing all realizations to collapse towards one that, indeed is coherent with the observed heads, but, as we will see later, it is biased with respect to the reference field.

As it has already been mentioned, the set of realizations improves as time passes and new piezometric head data are collected. This can be appreciated in Figure 6.9 where the piezometric heads predicted at piezometers \#6 and 

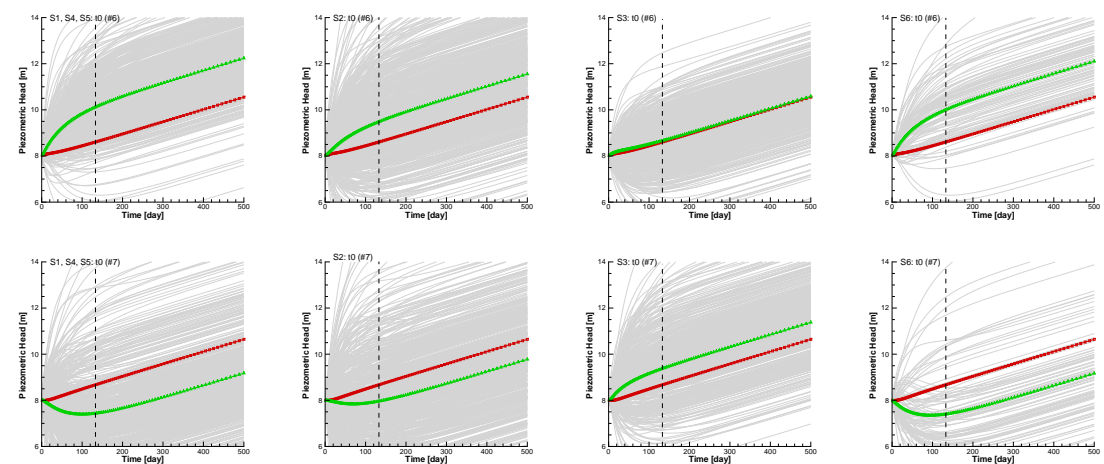

Figure 6.7. Evolution of piezometric head at piezometers \#6 (top row) and \#7 (bottom row) on the initial sets of realizations for the different scenarios. Scenarios S1, S4 and S5 share the same set of initial realizations (first column), scenarios S2, S3 and S6 are shown in the second, third and fourth columns, respectively. The gray lines correspond to the evolution in each realization. The red line is the piezometric evolution in the reference, and the green line is the time average of all gray lines. The vertical dashed lines indicate the end of the conditioning data.

\#7 with the ensembles of realizations generated at time zero, those generated after 10 time steps and those generated after 50 time steps are shown. The improvement with time is clear, although for the specific boundary conditions and stresses of this particular exercise, the improvement after time step 50 halts.

\subsubsection{Reproduction of conductivities}

From the previous section it is apparent that the final realizations are consistent, from a groundwater flow perspective, with the observed piezometric heads. The question now is how well they approximate the underlying hydraulic conductivity distribution.

Figure 6.10 shows the ensemble mean and ensemble variance computed locally through the initial ensembles of realizations for the different scenarios. Recall that all sets of initial realizations are generated with the hybrid method used to generate the reference field, that is, it combines multipoint geostatistics and Gaussian sequential simulation. Scenario S2 is unconditional, as a consequence the ensemble mean and variance are uniform and equal to the global mean and variance: even if each realization displays channel patterns as in the training image, the fact that no realization has information where any channel is renders realizations that, in average, are uninformative. Scenario S1 uses five conditioning points in sand close to the two vertical boundaries (where injection and production wells are placed) plus three more in shale in the center; we can notice the effect of condi- 

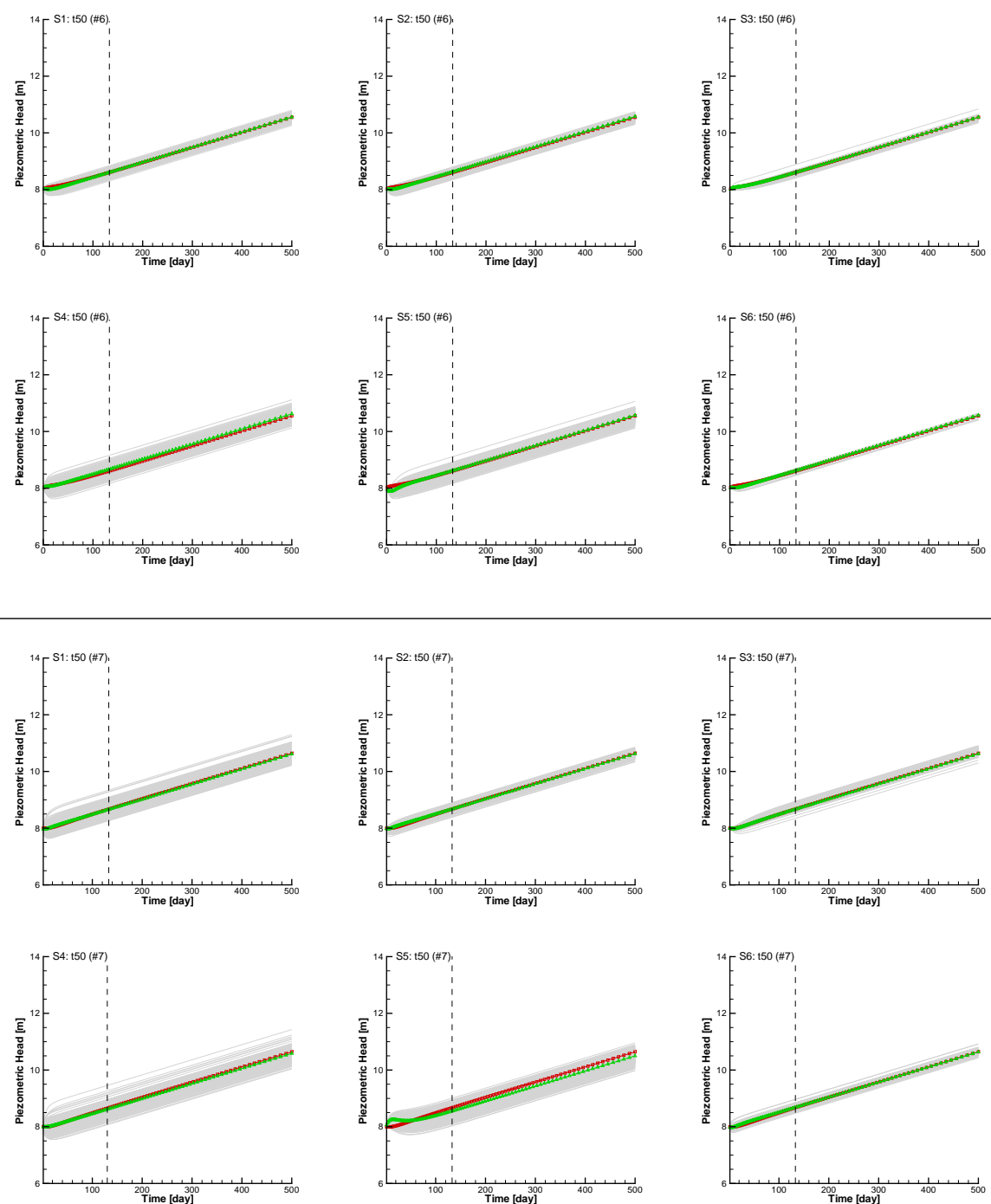

Figure 6.8. Evolution of piezometric head at piezometers \#6 (top two rows) and \#7 (bottom two rows) on the sets of realizations generated by iSS after conditioning to piezometric heads during the first 50 time steps (vertical dashed line) for the different scenarios. Meaning of lines is the same as previous figure. 

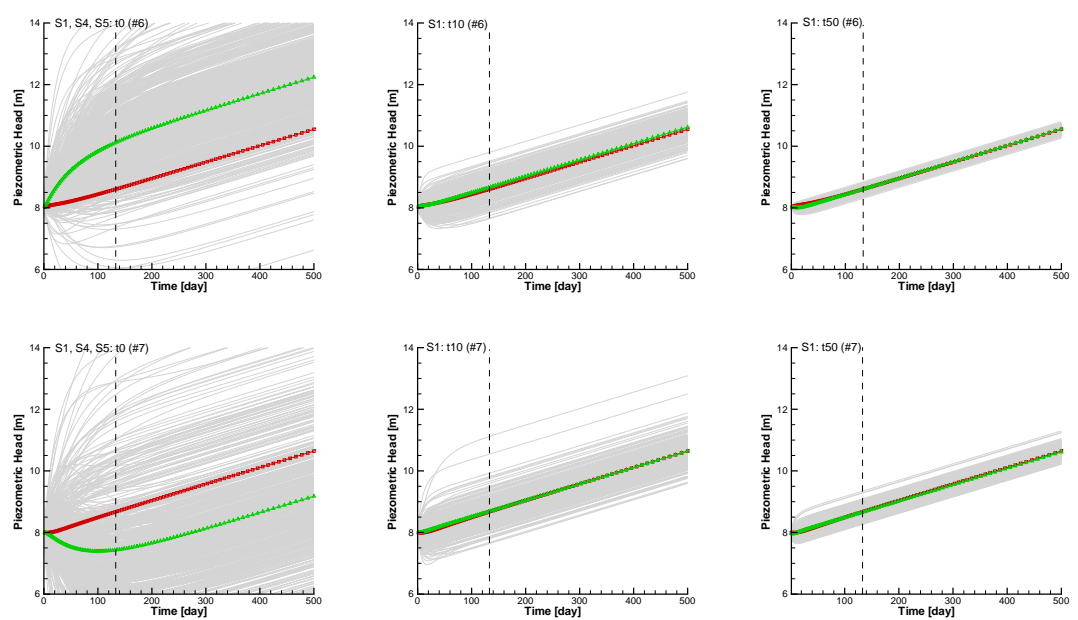

Figure 6.9. Evolution of piezometric head at piezometers \#6 (top row) and \#7 (bottom row) on the sets of realizations generated by iSS for scenario S1 at three different times: initial, after 10 time steps, and after 50 time steps. Meaning of lines is the same as previous figure.

tioning locally in the vicinity of the conditioning points, but we are still far from identifying the continuous channels present in the reference field. Scenario S6 yields virtually the same results as S1 with an ensemble of only 100 realizations. Scenario S3 has double the number of conditioning points well spread over all the aquifer producing realizations that, in average, start to identify the channels present in the reference.

Figure 6.11 shows the ensemble means and ensemble variances for all six scenarios after conditioning to piezometric heads for 50 time steps. As expected, the best results occur for scenario S3, the one with the largest amount of conditional information for both facies and piezometric head. It is the only scenario for which the ensemble mean captures the three channels and their geometry almost perfectly. Scenario S1 with a smaller number of facies conditional data does a good job, too, but introduces a link between the bottom two channels that does not exist in the reference and the edges of the channels are not as well defined as in S3. Scenario S6, which is the same as S1 but using only 100 realizations yields a channel distribution almost identical with $\mathrm{S} 1$ but it tends to overestimated the conductivity values, and the channels edges are even more noisy. The ensemble variance maps support the observations about the ensemble means. The variance for S3 indicates that there is some residual uncertainty in the edges of the channels, but otherwise the patterns are well captures in all realizations. This uncertainty about channel edges is larger in S1, for which there is also large uncertainty regarding that link area between the two bottom channels. 


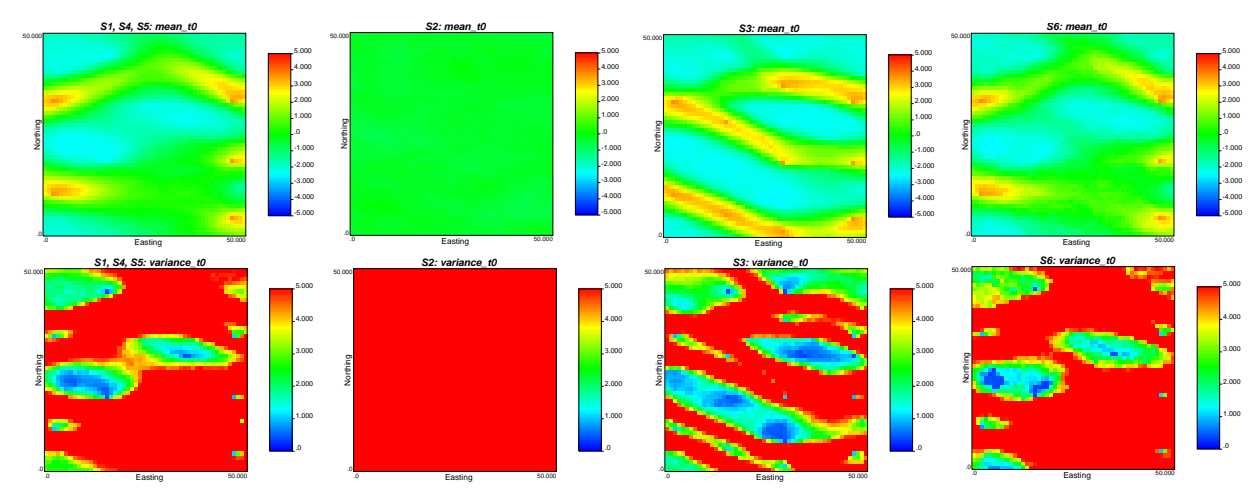

Figure 6.10. Ensemble mean (top row) and ensemble variance (bottom row) of logconductivity for the sets of initial realizations for the different scenarios. First column is for scenarios S1, S4 and S5, second column for S2, third column for S3, and fourth column for S6.

Whereas scenario S6 shows an overconfident ensemble variance, with values close to zero almost everywhere. This extreme reduction of the ensemble variance is due to an underestimation of the experimental covariance due to the use of a relatively small number of realizations. (This phenomenon is similar to the filter collapse observable in ensemble Kalman filtering when the ensemble size is small, and it is due to the same fact.)

The ensemble means and variances for scenarios S2 and S5 yield the same conclusions, after 50 time steps, the smaller number of conditioning data than in $\mathrm{S} 1$ (whether facies or piezometers) produce sets of realizations that start to delineate loosely the channels but that still display a lot of uncertainty. Had the algorithm kept working for another 50 time steps the results could have improved.

Finally, for implementation purposes, it is very important to retain a sufficiently large number of conditioning points within the search neighborhood for the algorithm to work. Reducing this number from 24 (for S1) to 8 (for $\mathrm{S} 4$ ) results in a mean map that misses the connectivity and a variance map with large values everywhere.

Figure 6.12 shows the evolution of the ensemble mean and ensemble variance maps for $\mathrm{S} 1$ with the initial realizations, with those obtained after 10 time steps, and with those obtained after 50 time steps. We can appreciate how the channels become better delineated as times passes, and how the ensemble variance reduces in the areas in which the mean map coincides with the reference.

The normal-score transform that is applied to transform the conductivities into Gaussian variates before the sequential simulation step of the algorithm is applied ensures that the final sets of conductivities have the bimodal characteristics of the reference field, as can be corroborated with 

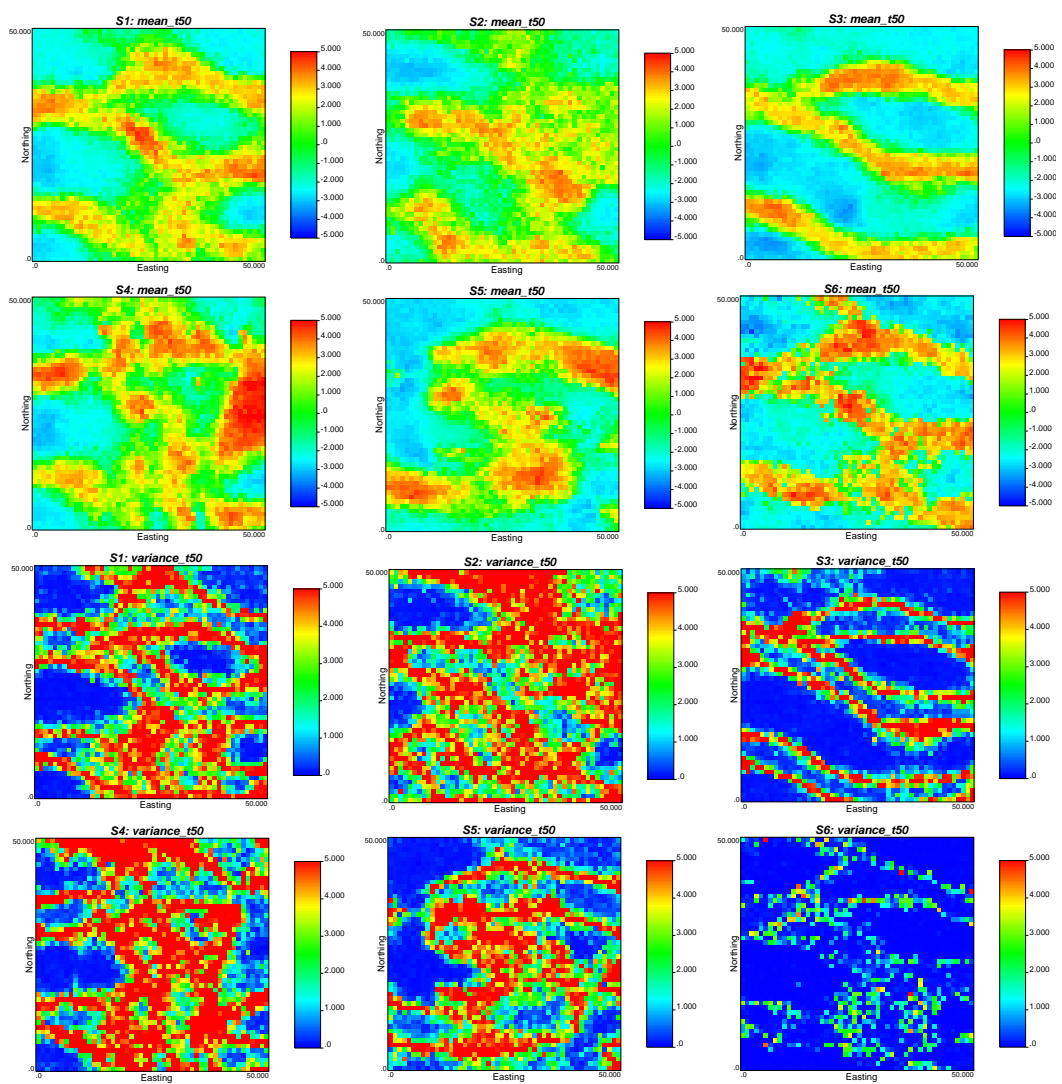

Figure 6.11. Ensemble mean (top two rows) and ensemble variance (bottom two rows) of logconductivity for the sets of realizations for the different scenarios obtained after 50 time steps.
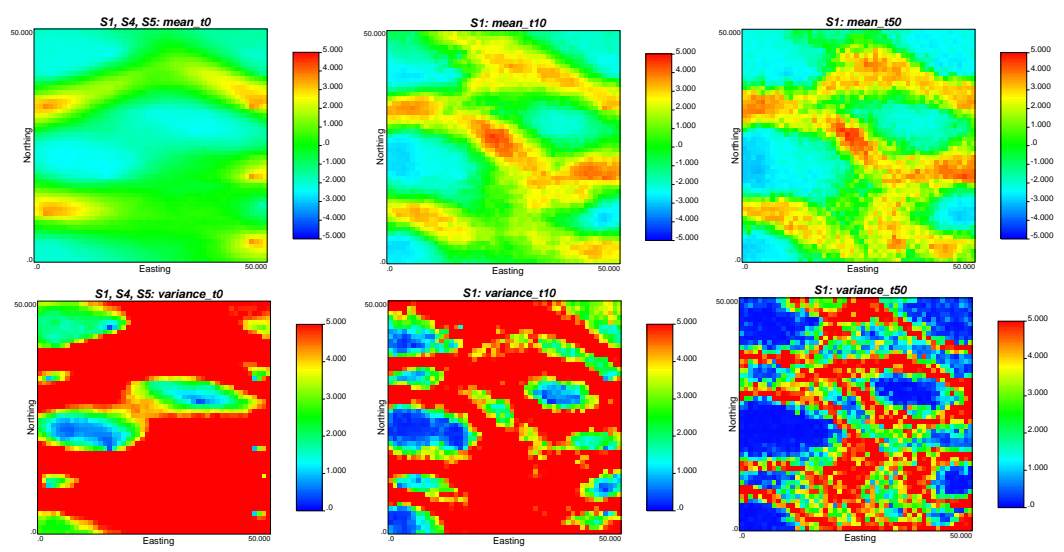

Figure 6.12. Ensemble mean (top row) and ensemble variance (bottom row) of logconductivity for the sets of realizations generated by iSS for scenario S1 at three different times: initial, after 10 time steps, and after 50 time steps. 
the histograms of the final realizations displayed in Figure 6.13. Overall all scenarios show the two modes, with the best reproduction of the reference histograms for those scenarios that we have already seen that perform best.
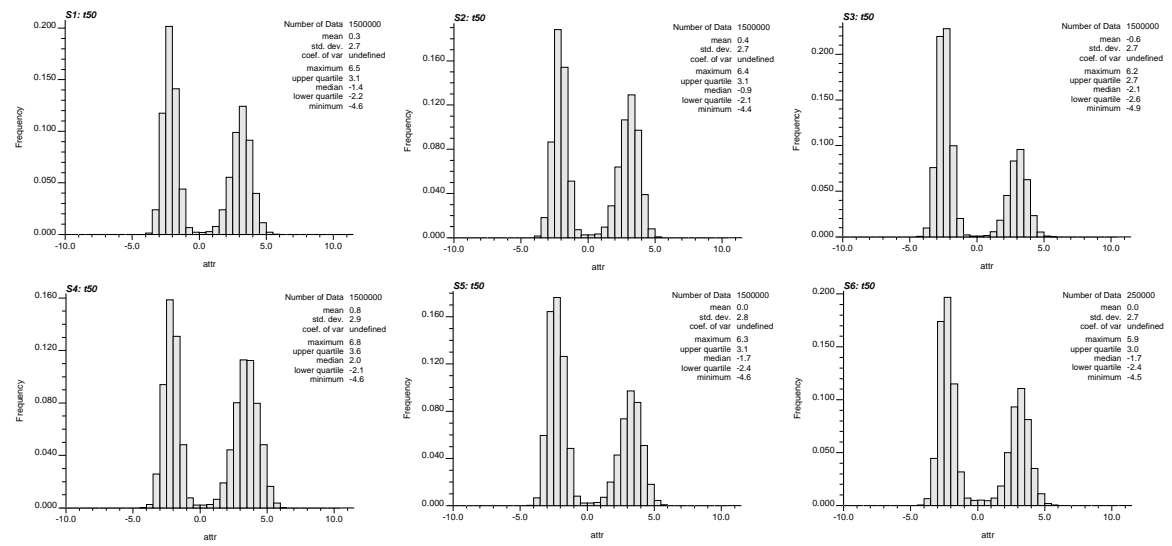

Figure 6.13. Histograms of logconductivity for the sets of realizations for the different scenarios obtained after 50 time steps.

The normal-score transform is particularly in this case a non-linear transformation which not only changes explicitly the marginal histograms of the variables involved, but in this case, it also affects, indirectly, the connectivity, as it can be seen in Figure 6.14. It is quite astonishing to compare the two columns of this figure; the mean of the normal-score transforms hardly displays any hint of channeling, and its variance is also quite distant in relative values and patterns from the variance map of the transformed log-conductivities. This can be explained because the local cumulative distribution functions are different from point to point, and for the conditioning effect of the piezometric heads.

We can quantify the goodness of the final sets of realizations by computing a single scalar measuring the accuracy with which the ensemble average matches the reference field and its precision as measured by the ensemble standard deviation. For this purpose, we define two metrics, the root mean square error (RMSE) and the average ensemble spread (ES) as:

$$
\begin{gathered}
R M S E=\sqrt{\frac{1}{N} \sum_{j=1}^{N}\left(\ln \left(K_{r e f}(j)\right)-\overline{\ln (K(j))}\right)^{2}}, \\
E S=\sqrt{\frac{1}{N} \sum_{j=1}^{N} \sigma_{j}^{2}},
\end{gathered}
$$




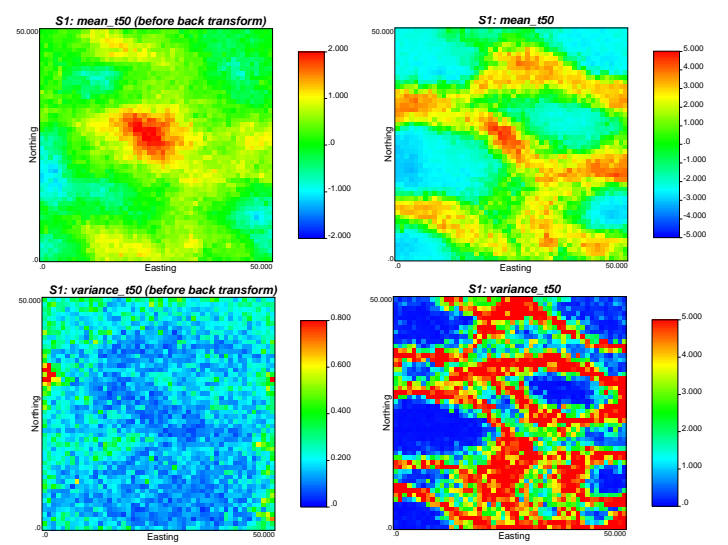

Figure 6.14. Ensemble mean (top row) and ensemble variance (bottom row) of the ensemble of normal scores of logconductivity as generated by the sequential simulation algorithm for scenario S1 after 50 time steps, and of the corresponding log-conductivity fields after backtransforming the normal-scores.

where $N$ is the number of model elements; $\ln (K)_{\text {ref }}(j)$ is the value of logconductivity in the reference field at node $j, \overline{\ln (K(j))}$ is the logconductivity ensemble mean at node $j ; \sigma_{i}$ is the ensemble variance at node $i$.

Figure 6.15 shows the evolution with time step of both the RMSE and the ES. Regarding the RMSE we can notice that, at the 50th time step, the best scenario is S3, as it was appreciated previously, and the worst scenario is S4, pinpointing the importance of good choice of the parameters controlling the sequential simulation step. As the number of facies conditioning data reduces, the RMSE increases (see the progression from S3 to S1 to S2). The second worst scenario is the one with the reduced number of piezometers used for the simulation, indicating the importance of having enough transient piezometric information for the algorithm to work. (Something that was also observed by Xu et al. (2013) in the context of the normalscore Ensemble Kalman filter.) Regarding the ensemble spread we notice how it decreases steadily in time for all scenarios, indicating a progressively less uncertain set of realizations; although, scenario S6, the one with only 100 realizations, shows a too dramatic decrease of the ES, which, in this case, indicates overconfidence since it is associated with a high value of the RMSE. This behavior of S6 is due to an underestimation of the experimental covariances, which are computed on a small number of realizations.

\subsubsection{Postaudit}

We have seen how the iSS is capable of generating ensembles of realizations which are conditioned to both conductivity data and piezometric head data. As a final check on the performance of the algorithm we perform a postaudit 


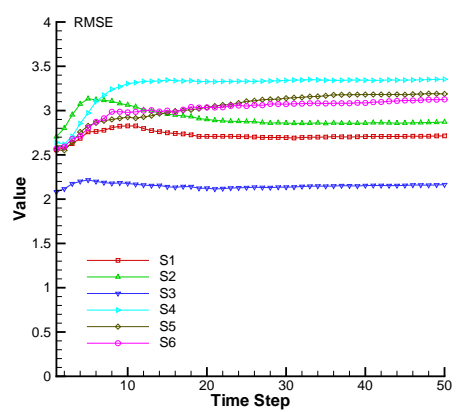

a

Figure 6.15. RMSE and ES of all the scenarios.

of all realizations and solve a solute transport problem, whose information had not been used for the generation of the realizations. For this purpose, keeping the same groundwater flow conditions as in the rest of the study, we model the release of solute at time zero from the vertical segment labeled "particle injection" in Figure 6.3 and located at $x=2.5 \mathrm{~m}$, and we monitor its arrival at the two control planes displayed in the same figure, located at $x=15 \mathrm{~m}$ and $x=45 \mathrm{~m}$. The total solute is distributed in 1000 particles, which are uniformly distributed along the segment and whose movement is tracked in the aquifer using the random-walk particle-tracking code RW3D by Fernàndez-Garcia et al. (2005). The solute is assumed to be inert, and the porosity of the aquifer is assumed to be uniform and equal to 0.3 .

Figure 6.16 shows a summary of the breakthrough curves (BTC) at the two control planes as computed in the initial sets of realizations used for the different scenarios; it shows, in red, the BTC for the reference field, in green the median of the BTCs computed timewise, and in black the $90 \%$ confidence interval as delimited by the $5 \%$ and $95 \%$ percentiles. The reference BTC has a very long tail, associated with all those particles which are introduced in the shale part of the release segment. Overall the BTCs show a large spread for the $90 \%$ confidence interval, which, in all cases, encloses the reference BTC. The spread is the smallest for scenario S3, what is coherent with the largest number of conditioning facies data used.

Figure 6.17 shows a summary of the same BTCs as the previous figure but now computed in the ensembles of realizations generated for all scenarios after 50 time steps. The spread of the BTCs has reduced considerably, although now some of the scenarios do not produce a $90 \%$ confidence interval fully enclosing the reference BTC.

At control plane 1, the one closest to the release segment, scenarios S1, S2, S3 and S4 behave similarly. Scenario S5 fails to capture the fast breakthrough of the first $75 \%$ of particles, what is related to the low conductivity values simulated in the upper right corner (see Figure 6.11), and which is 

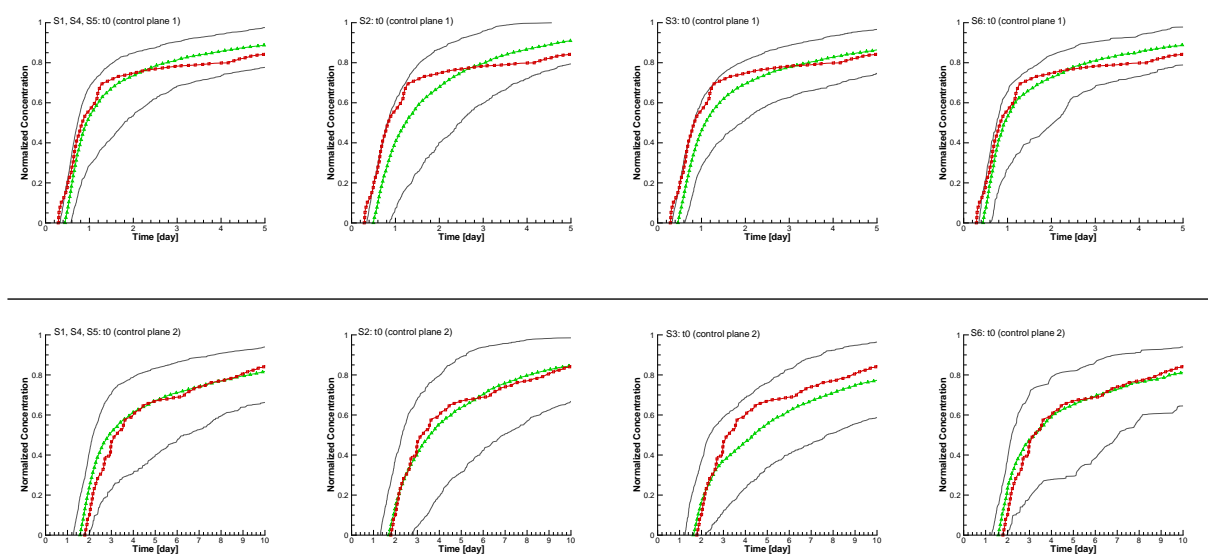

Figure 6.16. Solute breakthrough curves (BTC) at control planes 1 (top row) and 2 (bottom row) on the initial sets of realizations used in the different scenarios. The red line is the BTC in the reference, the green line is the median (computed timewise) of the BTCs from the different realizations, and the two black lines mark the $90 \%$ confidence interval bounded by the $5 \%$ and $95 \%$ percentiles.

due to the lowest number of conditioning piezometric head data overall, and specifically in the upper part of the aquifer. The results for S6 show the overconfident prediction already observed for conductivities (see Figure 6.11) and the bias associated to the highest departure from the reference field as measured by the RMSE.

At control plane 2 we can see that scenarios S1 and S2 behave similarly and with a good reproduction of the reference BTC. It is somehow surprising that scenario S3, which has been the best so far, produces BTCs that depart from the reference with a clear arrival in two bursts, a first one, which is faster than the reference, and a second one, slower than the reference. At this time we do not have an explanation for this behavior in relation with the proposed method. Scenarios S4 and S5 behave similarly (with slower travel times for S5) and display a larger spread. Finally, scenario S6 shows again its overconfidence and bias.

Figure 6.18 shows the evolution of the BTCs for scenario S1 in the initial realizations, in those generated after 10 time steps, and in those generated after 50 time steps. As before, we can see the reduction in their spread with time as a result of the conditioning to the observed piezometric heads.

\subsubsection{Discussion}

From the analysis of the results for the different scenarios we can conclude that the iSS is a method that can generate realizations of conductivity inverse conditioned on piezometric heads, that is, to generate conductivity 

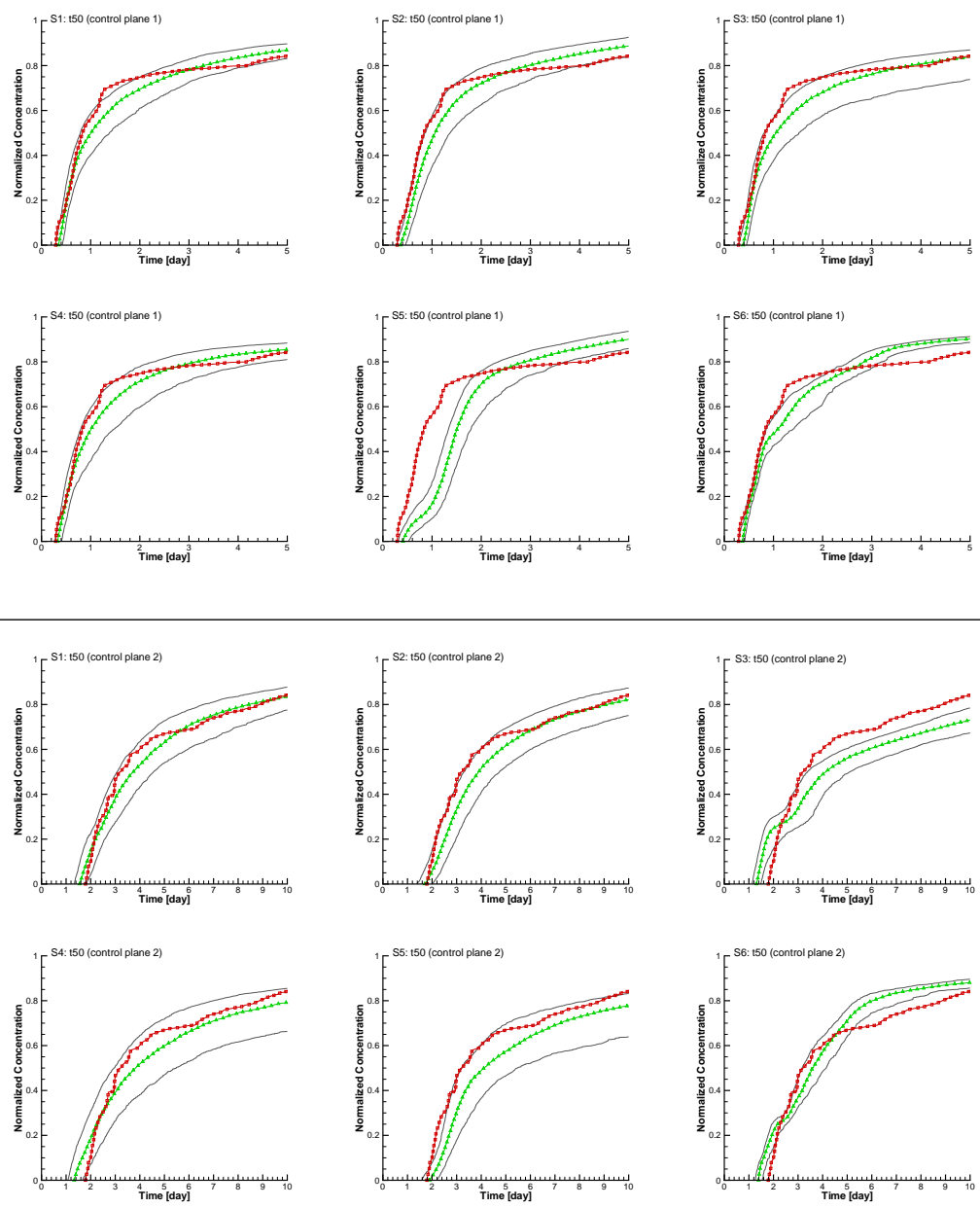

Figure 6.17. Solute breakthrough curves (BTC) at control planes 1 (top two rows) and 2 (bottom two rows) on the sets of realizations obtained for the different scenarios generated after 50 time steps. Meaning of lines is the same as in previous figure. 

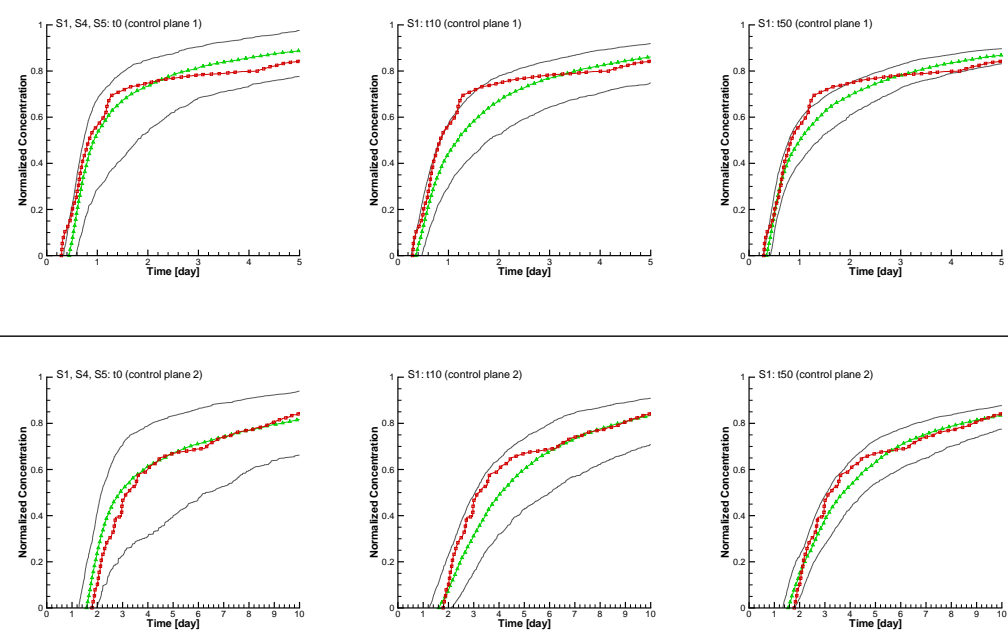

Figure 6.18. Solute breakthrough curves (BTC) at control planes 1 (top row) and 2 (bottom row) for scenario S1 on the initial set of realizations, those generated after 10 time steps, and those generated after 50 time steps. Meaning of lines is the same as in previous figure.

realizations that when used in a groundwater flow model will reproduce the observed heads. The method is not based in any optimization but rather in a covariance-based sequential simulation paradigm. The method has been demonstrated here for conductivities and piezometric heads, but it is applicable to any parameter and state variables related by a state equation.

From the different scenarios analyzed we can reach several conclusions, some affect parameters that can be decided by the user, and some parameters that are given. The first parameter that the user must define is how many realizations to generate: it is important to use a sufficiently large set of realizations to avoid the underestimation of the experimental covariances and cross-covariances. In this example 600 realizations were enough; 100 realizations resulted in the collapse of the final ensemble about a single realization. The problem of underestimation of the experimental covariances is also present in the implementation of the ensemble Kalman filter, and there it is solved using techniques of covariance inflation (e.g., Wang and Bishop, 2003; Anderson, 2007a; Li et al., 2009; Kurtz et al., 2011), those techniques could be a solution here, too. As a consequence of this requirement, the iSS is a method that cannot be used to generate a single conditional realization, there is always the need to generate a sufficiently large ensemble of realizations.

The standard sequential simulation algorithm has three key aspects that must be decided by the user, which are of importance for the iSS, too: the size and shape of the search neighborhood used for the building of the local 
conditional probability distribution, the maximum number of points (and their spatial distribution) to retain within the search neighborhood, and the variables to simulate. Regarding the size and shape of the search neighborhood, this is related with the correlation structure of the parameter field, in our example, the overall horizontal correlation of conductivity is much larger than the size of the aquifer due to the presence of the horizontal channels, while the vertical correlation is smaller. We chose a large search neighborhood, of $25 \mathrm{~m}$ radius, so that, if centered in the aquifer it covers most of it. Regarding the maximum number of points to retain within the search neighborhood, the results show that a large number is necessary for a good reproduction of the conductivity patters. We have not analyzed the spatial disposition of these retained and we have chosen always the closest points, but it would be interesting to analyze the impact of distributing these data by quadrants, or by type of conditioning variable. And regarding the variables to simulate, we can choose to generate only conductivities conditioned on both conductivities and piezometric heads, or to generate both conductivities and piezometric heads simultaneously. If we choose to generate only one variable then as the grid is being generated and the number of simulated nodes increases, the conditioning data retained within the search neighborhood contains less and less number of piezometric heads (since they are only 16 or 25 values at all times, while the number of conductivity conditioning data reaches 2499 values for the simulation of the last node); whereas if we generate both variables simultaneously we can argue that the cross-correlation between conductivities and piezometric heads are persistent throughout all the simulation since until the simulation of last nodes there will always be data within the search neighborhood of both variables. In this study, we have generated only conductivities; however, we have tried the alternative of generating both variables at the same time, and although the results are not shown here we can say that the results are very similar. Therefore, it is preferable to generate only the variable of interest since it is faster.

There are other inputs that cannot be controlled by the user, such as the number of conductivity and piezometric head conditioning data. The previous analysis gives the obvious result that the larger the number of conditioning data of either variable, the better the final outcome.

Finally, there is a critical parameter that we have not mentioned before but which controls the feasibility of the method, which is a nugget effect that must be added to the experimental covariances and cross-covariances. It is well known that the experimental covariances are not positive definite and, therefore, its direct use in the simple kriging equations to compute the kriging mean and kriging variance during the sequential simulation algorithm could yield singular kriging matrices or, if the kriging equations can be solved, the resulting kriging variance may be negative. To solve this problem we must first yield the experimental covariances positive definite. 
Yao and Journel (1998) propose a method based in fast Fourier transforms to solve this problem. A less elegant, but simpler approach, is to add a nugget effect to the computed experimental covariances. In our study we had to add a nugget effect equal to $1 \%$ of the total variance to the diagonal of the experimental covariance matrices to render them positive definite. This a parameter that must be fine tuned, searching for the smallest value that will make the sequential simulation possible. 


\section{7 \\ Conclusions}

\subsection{Summary}

The work of this thesis is aimed to fulfill the main objectives described in Chapter 1.1. Some of the conclusions derived are the following:

- A parallel implementation of the EnKF can reduce significantly the computing time of the standard EnKF. The parallelization runs with higher efficiency for large size models and large ensemble sizes than for small ones. Typically, the efficiency can remain over 0.40 when using up to 12 processors for the two tests performed using 1200 realizations.

- We have shown that, even with no information about the spatial heterogeneity of conductivity but its univariate marginal distribution, the NS-EnKF can get a proper characterization of conductivities via assimilating enough transient piezometric heads. Besides, we have also shown that covariance localization and covariance inflation techniques are necessary to eliminate the effect of spurious correlations and to reduce the filter inbreeding.

- We have analyzed in depth the algorithm proposed by (Hu et al., 2013) to find that it fails in the characterization of a channelized bimodal hydraulic conductivity field. The approach by $\mathrm{Hu}$ is based on the updating of an initially uncorrelated field of normal Gaussian deviates, we think that the poor performance of the method is due to the very weak (almost non-existent) correlation between the variables being updated and the state variables; based on this premise, we propose the 
updating of a field of correlated Gaussian deviates and we prove that the results with the new updating variables is far superior to the one proposed by $\mathrm{Hu}$ and capable of a proper estimation for non-Gasssian bimodal hydraulic conductivities.

- Finally, blending some of the ideas underlying multivariate Gaussian sequential simulation and ensemble Kalman filtering, we have developed a new stochastic inverse modeling method, which we have termed inverse sequential simulation (iSS) that can generate realizations of hydraulic conductivity inverse-conditioned on piezometric heads. The main steps of the iSS are as follows: first, build normal score transform functions and transform all the conductivity values of all the realizations in all the grid nodes into Gaussian distributed values; and then, use these transformed realizations to compute an experimental, non-stationary conditional cross-covariance between transformed conductivities and piezometric heads, and auto-covariance of both transformed conductivities and piezometric heads; next, a standard multivariate sequential Gaussian conditional simulation algorithm is used to generate a new ensemble of transformed conductivity realizations; and then back-transform these transformed conductivity values and go to next time step. We have found that iSS compares favorably with the NS-EnKF, although it still needs some improvements to make it computationally attractive.

\subsection{Suggestions for Future Research}

After reviewing the whole work in this thesis, we find there are still some issues deserving to study,

- Jointly estimate state parameters conditioning to several types of data. In this thesis, the estimation of hydraulic conductivities is via assimilating the transient piezometric head data only. In fact, for real flow or transport modeling, transient piezometric head is not the only state variable we can get, but also some other state variables, such as concentration, or groundwater temperature, which can be jointly assimilated to estimate the hydraulic conductivities. Moreover, these state variables can also be used to jointly estimate other uncertain state parameters, such as leakage coefficient and porosity.

- Propose a parallelized iSS algorithm to reduce the computational time. The result of chapter 5 shows that although the iSS is an efficient approach that is capable of coping with non-Gaussian distribution, the needed CPU time is still high and its application would be difficult for the modelings with large ensemble size or large model sizes. 
- Demonstrate the NS-EnKF or the iSS to real case studies.

The cases we employed in this thesis are all synthetic so that we can control all parameters and where uncertainties are coming from. The application of these, apparently powerful, methods to read data sets sometimes fail. It is necessary to carry out those applications and to analyze the reasons of failure and find solutions. 


\section{Bibliography}

Aanonsen, S., Nævdal, G., Oliver, D., Reynolds, A., Vallès, B., 2009. The ensemble kalman filter in reservoir engineering-a review. SPE Journal 14, 393-412.

Alcolea, A., Carrera, J., Medina, A., 2006. Pilot points method incorporating prior information for solving the groundwater flow inverse problem. Advances in Water Resources 29, 1678-1689.

Anderson, J., 2007a. An adaptive covariance inflation error correction algorithm for ensemble filters. Tellus A 59, 210-224.

Anderson, J., 2007b. Exploring the need for localization in ensemble data assimilation using a hierarchical ensemble filter. Physica D: Nonlinear Phenomena 230, 99-111.

Apte, A., Hairer, M., Stuart, A., Voss, J., 2007. Sampling the posterior: An approach to non-gaussian data assimilation. Physica D: Nonlinear Phenomena 230, 50-64.

Arpat, G.B., Caers, J., 2007. Conditional simulation with patterns. Mathematical Geology 39, 177-203.

Attia, A., Sandu, A., 2014. A sampling filter for non-gaussian data assimilation. arXiv preprint arXiv:1403.7137 .

Béal, D., Brasseur, P., Brankart, J., Ourmières, Y., Verron, J., et al., 2010. Characterization of mixing errors in a coupled physical biogeochemical model of the north atlantic: implications for nonlinear estimation using gaussian anamorphosis. Ocean Science .

Bergemann, K., Reich, S., 2010. A localization technique for ensemble kalman filters. Quarterly Journal of the Royal Meteorological Society 136, 701-707.

Bertino, L., Evensen, G., Wackernagel, H., 2003a. Sequential data assimilation techniques in oceanography. International Statistical Review 71, $223-241$. 
Bertino, L., Hollard, A., Evensen, G., Wackernagel, H., 2003b. An ensemble kalman filter for non-gaussian variables, in: EGS-AGU-EUG Joint Assembly, p. 5171.

Blöschl, G., Reszler, C., Komma, J., 2008. A spatially distributed flash flood forecasting model. Environmental Modelling \& Software 23, 464-478.

Bocquet, M., Pires, C., Wu, L., 2010. Beyond gaussian statistical modeling in geophysical data assimilation. Monthly Weather Review 138, 29973023.

Boucher, A., 2009. Considering complex training images with search tree partitioning. Computers \& Geosciences 35, 1151-1158.

Burgers, G., van Leeuwen, P., Evensen, G., Instituut, K.N.M., 1998. Analysis scheme in the ensemble kalman filter. Monthly weather review 126, $1719-1724$.

Capilla, J.E., Llopis-Albert, C., 2009. Gradual conditioning of non-gaussian transmissivity fields to flow and mass transport data: 1 . theory. Journal of hydrology 371, 66-74.

Capilla, J.E., Rodrigo, J., Gómez-Hernández, J.J., 1999. Simulation of nonGaussian transmissivity fields honoring piezometric data and integrating soft and secondary information. Mathematical Geology 31, 907-927.

Chang, H., Zhang, D., Lu, Z., 2010. History matching of facies distribution with the enkf and level set parameterization. Journal of Computational Physics 229, 8011-8030.

Chen, R., Liu, J., 2000. Mixture kalman filters. Journal of the Royal Statistical Society: Series B (Statistical Methodology) 62, 493-508.

Chen, Y., Oliver, D., 2010a. Cross-covariances and localization for enkf in multiphase flow data assimilation. Computational Geosciences 14, 579601.

Chen, Y., Oliver, D., 2010b. Parameterization techniques to improve mass conservation and data assimilation for ensemble kalman filter, in: SPE Western Regional Meeting.

Chen, Y., Oliver, D., Zhang, D., 2009. Data assimilation for nonlinear problems by ensemble kalman filter with reparameterization. Journal of Petroleum Science and Engineering 66, 1-14.

Chen, Y., Zhang, D., 2006. Data assimilation for transient flow in geologic formations via ensemble kalman filter. Advances in Water Resources 29, $1107-1122$. 
Chugunova, T.L., Hu, L.Y., 2008. Multiple-point simulations constrained by continuous auxiliary data. Mathematical geosciences 40, 133-146.

De Marsily, G., Lavedan, G., Boucher, M., Fasanino, G., 1984. Interpretation of interference tests in a well field using geostatistical techniques to fit the permeability distribution in a reservoir model. Geostatistics for natural resources characterization, Part 2, 831-849.

Delbari, M., Afrasiab, P., Loiskandl, W., 2009. Using sequential gaussian simulation to assess the field-scale spatial uncertainty of soil water content. Catena 79, 163-169.

Deutsch, C., Journel, A., 1998a. Gslib: Geostatistical software library and user's guide. New York 369.

Deutsch, C.V., Journel, A.G., 1992. Geostatistical software library and user\&s guide. volume 1996. Oxford university press New York.

Deutsch, C.V., Journel, A.G., 1998b. GSLIB, Geostatistical Software Library and User's Guide. Oxford University Press, New York. second edition.

Devegowda, D., Arroyo-Negrete, E., Datta-Gupta, A., 2010. Flow relevant covariance localization during dynamic data assimilation using enkf. Advances in Water Resources 33, 129-145.

Dewaraja, Y., Ljungberg, M., Majumdar, A., Bose, A., Koral, K., 2002. A parallel monte carlo code for planar and spect imaging: implementation, verification and applications in 131i spect. Computer methods and programs in biomedicine $67,115-124$.

Dovera, L., Della Rossa, E., 2011. Multimodal ensemble kalman filtering using gaussian mixture models. Computational Geosciences 15, 307-323.

Dowell, D.C., Zhang, F., Wicker, L.J., Snyder, C., Crook, N.A., 2004. Wind and temperature retrievals in the 17 may 1981 arcadia, oklahoma, supercell: Ensemble kalman filter experiments. Monthly weather review 132, 1982-2005.

Evensen, G., 1994. Sequential data assimilation with a nonlinear quasigeostrophic model using monte carlo methods to forecast error statistics. J. Geophys. Res 99, 143-10.

Evensen, G., 2003. The ensemble kalman filter: Theoretical formulation and practical implementation. Ocean dynamics 53, 343-367.

Fernàndez-Garcia, D., Illangasekare, T., Rajaram, H., 2005. Differences in the scale dependence of dispersivity and retardation factors estimated 
from forced-gradient and uniform flow tracer tests in three-dimensional physically and chemically heterogeneous porous media. Water resources research 41, W03012.

Fiori, A., Indelman, P., Dagan, G., 1998. Correlation structure of flow variables for steady flow toward a well with application to highly anisotropic heterogeneous formations. Water resources research 34, 699-708.

Franssen, H., Gómez-Hernández, J., Sahuquillo, A., 2003. Coupled inverse modelling of groundwater flow and mass transport and the worth of concentration data. Journal of Hydrology 281, 281-295.

Franssen, H., Kinzelbach, W., 2008. Real-time groundwater flow modeling with the ensemble kalman filter: Joint estimation of states and parameters and the filter inbreeding problem. Water Resources Research 44, W09408.

Friedel, M.J., Iwashita, F., 2013. Hybrid modeling of spatial continuity for application to numerical inverse problems. Environmental Modelling \& Software 43, 60-79.

Froidevaux, R., 1993. Probability field simulation, in: Geostatistics Troia 92. Springer, pp. $73-83$.

Fu, J., Jaime Gómez-Hernández, J., 2009. Uncertainty assessment and data worth in groundwater flow and mass transport modeling using a blocking markov chain monte carlo method. Journal of Hydrology 364, 328-341.

Gaspari, G., 1999. Construction of correlation functions in two and three dimensions. Quart. J. Roy. Meteor. Soc. 125, 723-757.

Gómez-Hernández, J., Wen, X.H., 1994. Probabilistic assessment of travel times in groundwater modeling. Stochastic Hydrology and Hydraulics 8, $19-55$.

Gómez-Hernández, J.J., Journel, A.G., 1993. Joint sequential simulation of multi-Gaussian fields. Geostatistics Troia 92, 85-94.

Gómez-Hernández, J.J., Journel, A.G., 1993. Joint sequential simulation of Multi-Gaussian fields, in: Soares, A. (Ed.), Geostatistics Tróia '92, Kluwer Academic Publishers, Dordrecht. pp. 85-94.

Gómez-Hernández, J.J., Wen, X.H., 1998. To be or not to be multiGaussian: A reflection in stochastic hydrogeology. Advances in Water Resources 21, $47-61$.

Gómez-Hernánez, J., Sahuquillo, A., Capilla, J., 1997. Stochastic simulation of transmissivity fields conditional to both transmissivity and piezometric data-1. theory. Journal of Hydrology(Amsterdam) 203, 167-174. 
Goovaerts, P., 1997. Geostatistics for Natural Resources Evaluation. Oxford University Press, New York.

Gordon, N., Salmond, D., Smith, A., 1993. Novel approach to nonlinear/non-gaussian bayesian state estimation, in: Radar and Signal Processing, IEE Proceedings F, IET. pp. 107-113.

Greybush, S., Kalnay, E., Miyoshi, T., Ide, K., Hunt, B., 2011. Balance and ensemble kalman filter localization techniques. Monthly Weather Review $139,511-522$.

Gu, Y., Oliver, D., 2006. The ensemble kalman filter for continuous updating of reservoir simulation models. TRANSACTIONS-AMERICAN SOCIETY OF MECHANICAL ENGINEERS JOURNAL OF ENERGY RESOURCES TECHNOLOGY 128, 79.

Gu, Y., Oliver, D., 2007. An iterative ensemble kalman filter for multiphase fluid flow data assimilation. SPE Journal 12, 438-446.

Guardiano, F., Srivastava, R., 1993a. Multivariate geostatistics: beyond bivariate moments, in: Soares, A. (Ed.), Geostatistics-Troia, Kluwer Academic Publ, Dordrecht. pp. 133-144.

Guardiano, F.B., Srivastava, R.M., 1993b. Multivariate geostatistics: Beyond bivariate models, in: Soares, A. (Ed.), Geostatistics Tróia '92, volume 1, Kluwer. pp. 133-144.

Hamill, T., Whitaker, J., Snyder, C., 2001. Distance-dependent filtering of background error covariance estimates in an ensemble kalman filter. Monthly Weather Review 129, 2776-2790.

Hantush, M., 1997. Estimation of spatially variable aquifer hydraulic properties using kalman filtering. Journal of Hydraulic Engineering 123, 1027.

Harbaugh, A., et al., 2000. MODFLOW-2000, the US Geological Survey modular ground-water model: User guide to modularization concepts and the ground-water flow process. US Geological Survey.

Hendricks Franssen, H.J., Kinzelbach, W., 2009. Ensemble kalman filtering versus sequential self-calibration for inverse modelling of dynamic groundwater flow systems. Journal of Hydrology 365, 261-274.

Honarkhah, M., Caers, J., 2010. Stochastic simulation of patterns using distance-based pattern modeling. Mathematical Geosciences 42, 487-517.

Houtekamer, P., Mitchell, H., 1998. Data assimilation using an ensemble kalman filter technique. Monthly Weather Review 126, 796-811. 
Houtekamer, P., Mitchell, H., 2001. A sequential ensemble kalman filter for atmospheric data assimilation. Monthly Weather Review 129, 123-137.

$\mathrm{Hu}$, L., 2000. Gradual deformation and iterative calibration of gaussianrelated stochastic models. Mathematical Geology 32, 87-108.

Hu, L., Zhao, Y., Liu, Y., Scheepens, C., Bouchard, A., 2012a. Updating multipoint simulations using the ensemble kalman filter. Computers \& Geosciences .

Hu, L., Zhao, Y., Liu, Y., Scheepens, C., Bouchard, A., 2013. Updating multipoint simulations using the ensemble kalman filter. Computers \& Geosciences 51, 7-15.

Hu, L.Y., Zhao, Y., Liu, Y., Scheepens, C., Bouchard, A., 2012b. Updating multipoint simulatings using the ensemble kalman filter. Computers \& Geosciences, in press.

Jafarpour, B., Khodabakhshi, M., 2011. A probability conditioning method (PCM) for nonlinear flow data integration into multipoint statistical facies simulation. Mathematical Geosciences 43, 133-164.

Kalman, R., et al., 1960. A new approach to linear filtering and prediction problems. Journal of basic Engineering 82, 35-45.

Karri, R.R., Wang, X., Gerritsen, H., 2014. Ensemble based prediction of water levels and residual currents in singapore regional waters for operational forecasting. Environmental Modelling \& Software 54, 24-38.

Keppenne, C., 2000. Data assimilation into a primitive-equation model with a parallel ensemble kalman filter. MONTHLY WEATHER REVIEW-USA 128, 1971-1981.

Keppenne, C., Rienecker, M., 2002a. Development and initial testing of a parallel ensemble kalman filter for the poseidon isopycnal ocean general circulation model. Mon. Wea. Rev 130, 2951-2965.

Keppenne, C., Rienecker, M., 2002b. Initial testing of a massively parallel ensemble kalman filter with the poseidon isopycnal ocean general circulation model. Monthly Weather Review(0027-0644) 130, 2951-2965.

Keppenne, C., Rienecker, M., 2003. Assimilation of temperature into an isopycnal ocean general circulation model using a parallel ensemble kalman filter. Journal of marine systems 40, 363-380.

Knudby, C., Carrera, J., 2005. On the relationship between indicators of geostatistical, flow and transport connectivity. Advances in Water Resources 28, 405-421. 
Kurtz, A., Lugolobi, F., Salvucci, G., 2011. Germanium-silicon as a flow path tracer: Application to the rio icacos watershed. Water Resources Research 47, W06516.

Leng, C., Yeh, H., 2003. Aquifer parameter identification using the extended kalman filter. Water resources research 39, 1062.

Li, G., Reynolds, A., 2007. An iterative ensemble kalman filter for data assimilation, in: SPE Annual Technical Conference and Exhibition.

Li, H., Kalnay, E., Miyoshi, T., 2009. Simultaneous estimation of covariance inflation and observation errors within an ensemble kalman filter. Quarterly Journal of the Royal Meteorological Society 135, 523-533.

Li, L., Srinivasan, S., Zhou, H., Gómez-Hernández, J.J., 2013. Simultaneous estimation of geologic and reservoir state variables within an ensemblebased multiple-point statistic framework. Mathematical Geosciences , 127.

Li, L., Zhou, H., Franssen, H., Gómez-Hernández, J., 2011. Groundwater flow inverse modeling in non-multigaussian media: performance assessment of the normal-score ensemble kalman filter. Hydrol. Earth Syst. Sci. Discuss 8, 6749-6788.

Li, L., Zhou, H., Gómez-Hernández, J., Hendricks Franssen, H., 2012a. Jointly mapping hydraulic conductivity and porosity by assimilating concentration data via ensemble kalman filter. Journal of Hydrology .

Li, L., Zhou, H., Hendricks Franssen, H., Gómez-Hernández, J., 2012b. Groundwater flow inverse modeling in non-multigaussian media: performance assessment of the normal-score ensemble kalman filter. Hydrology and Earth System Sciences 16, 573 .

Li, L., Zhou, H., Hendricks Franssen, H.J., Gómez-Hernández, J.J., 2012c. Modeling transient groundwater flow by coupling ensemble kalman filtering and upscaling. Water Resources Research 48.

Li, X., Lei, Z., White, C., Allen, G., Qin, G., Tsai, F., 2007. Grid-enabled ensemble subsurface modeling, in: Proceedings of The 19th IASTED International Conference on Parallel and Distributed Computing and Systems (PDCS 2007).

Liang, X., Zheng, X., Zhang, S., Wu, G., Dai, Y., Li, Y., 2011. Maximum likelihood estimation of inflation factors on error covariance matrices for ensemble kalman filter assimilation. Quarterly Journal of the Royal Meteorological Society 138, 263-273. 
Liu, N., Oliver, D., 2005. Critical evaluation of the ensemble kalman filter on history matching of geologic facies, in: SPE Reservoir Simulation Symposium.

van Loon, M., Builtjes, P.J., Segers, A., 2000. Data assimilation of ozone in the atmospheric transport chemistry model lotos. Environmental Modelling \& Software 15, 603-609.

Losa, S., Kivman, G., Schröter, J., Wenzel, M., 2003. Sequential weak constraint parameter estimation in an ecosystem model. Journal of Marine Systems 43, 31-49.

Mariethoz, G., Renard, P., Straubhaar, J., 2010. The direct sampling method to perform multiple-point geostatistical simulations. Water Resources Research 46, W11536.

McDonald, M., Harbaugh, A., 1984. A modular three-dimensional finitedifference ground-water flow model. Scientific Publications Company.

McDonald, M., Harbaugh, A., 1988. A modular three-dimensional finitedifference ground-water flow model .

McElhoe, B.A., 1966. An assessment of the navigation and course corrections for a manned flyby of mars or venus, in: IEEE Transactions on Aerospace and Electronic Systems, Supplement, IEEE. pp. 613-623.

Mirowski, P.W., Tetzlaff, D.M., Davies, R.C., McCormick, D.S., Williams, N., Signer, C., 2009. Stationarity scores on training images for multipoint geostatistics. Mathematical geosciences 41, 447-474.

Moradkhani, H., Sorooshian, S., Gupta, H., Houser, P., 2005. Dual stateparameter estimation of hydrological models using ensemble kalman filter. Advances in Water Resources 28, 135-147.

Mustapha, H., Dimitrakopoulos, R., 2010. High-order stochastic simulation of complex spatially distributed natural phenomena. Mathematical Geosciences 42, 457-485.

Nan, T., Wu, J., 2011. Groundwater parameter estimation using the ensemble kalman filter with localization. Hydrogeology Journal 19, 547-561.

Neuweiler, I., Cirpka, O., 2005. Homogenization of richards equation in permeability fields with different connectivities. Water resources research 41, W02009.

Oliver, D., Cunha, L., Reynolds, A., 1997. Markov chain monte carlo methods for conditioning a permeability field to pressure data. Mathematical Geology 29, 61-91. 
Pardo-Igúzquiza, E., Dowd, P., 2003. Connec3d: a computer program for connectivity analysis of $3 \mathrm{~d}$ random set models. Computers \& geosciences $29,775-785$.

RamaRao, B., LaVenue, A., De Marsily, G., Marietta, M., 1995. Pilot point methodology for automated calibration of an ensemble of conditionally simulated transmissivity fields: 1 . theory and computational experiments. Water Resources Research 31, 475-493.

Reich, S., 2011. A gaussian-mixture ensemble transform filter. Quarterly Journal of the Royal Meteorological Society 138, 222-233.

Sarma, P., Chen, W., 2009. Generalization of the ensemble kalman filter using kernels for nongaussian random fields, in: SPE Reservoir Simulation Symposium.

Schöniger, A., Nowak, W., Franssen, H., 2012. Parameter estimation by ensemble kalman filters with transformed data: Approach and application to hydraulic tomography. Water Resources Research 48, W04502.

Simon, E., Bertino, L., 2009. Application of the gaussian anamorphosis to assimilation in a 3 -d coupled physical-ecosystem model of the north atlantic with the enkf: a twin experiment. Ocean Sci 5, 495-510.

Simon, E., Bertino, L., 2012. Gaussian anamorphosis extension of the denkf for combined state parameter estimation: Application to a $1 \mathrm{~d}$ ocean ecosystem model. Journal of Marine Systems 89, 1-18.

Stauffer, D., Aharony, A., 1994. Introduction to percolation theory. Taylor and Francis, London. 181pp.

Stordal, A., Karlsen, H., Nævdal, G., Skaug, H., Vallès, B., 2011. Bridging the ensemble kalman filter and particle filters: the adaptive gaussian mixture filter. Computational Geosciences 15, 293-305.

Strébelle, S., 2000. Sequential simulation drawing structures from training images. Ph.D. thesis. Stanford University. 187pp.

Strebelle, S., 2002. Conditional simulation of complex geological structures using multiple-point statistics. Mathematical Geology 34, 1-21.

Sun, A., Morris, A., Mohanty, S., 2009. Sequential updating of multimodal hydrogeologic parameter fields using localization and clustering techniques. Water Resources Research 45, W07424.

Tavakoli, R., Pencheva, G., Wheeler, M., 2011. Multi-level parallelization of ensemble kalman filter for reservoir history matching, in: SPE Reservoir Simulation Symposium. 
Valstar, J.R., McLaughlin, D.B., Te Stroet, C., van Geer, F.C., 2004. A representer-based inverse method for groundwater flow and transport applications. Water Resources Research 40.

Van Leeuwen, P., 2009. Particle filtering in geophysical systems. Monthly Weather Review 137, 4089-4114.

Wang, X., Bishop, C., 2003. A comparison of breeding and ensemble transform kalman filter ensemble forecast schemes. Journal of the atmospheric sciences $60,1140-1158$.

Wang, Y., Li, G., Reynolds, A., 2010. Estimation of depths of fluid contacts by history matching using iterative ensemble-kalman smoothers. SPE Journal 15, 509-525.

Wen, X., Chen, W., 2005. Some practical issues on real-time reservoir model updating using ensemble kalman filter, in: International Petroleum Technology Conference.

Wen, X., Chen, W., 2006. Real-time reservoir model updating using ensemble kalman filter with confirming option. SPE Journal 11, 431-442.

Wen, X., Deutsch, C., Cullick, A., 2002. Construction of geostatistical aquifer models integrating dynamic flow and tracer data using inverse technique. Journal of Hydrology 255, 151-168.

Western, A., Blöschl, G., Grayson, R., 2001. Toward capturing hydrologically significant connectivity in spatial patterns. Water Resources Research $37,83-97$.

Xu, T., Jaime Gómez-Hernández, J., Li, L., Zhou, H., 2012. Parallelized ensemble kalman filter for hydraulic conductivity characterization. Computers \& Geosciences, http://dx.doi.org/10.1016/j.cageo.2012.10.007.

Xu, T., Jaime Gómez-Hernández, J., Zhou, H., Li, L., 2013. The power of transient piezometric head data in inverse modeling: An application of the localized normal-score enkf with covariance inflation in a heterogenous bimodal hydraulic conductivity field. Advances in Water Resources .

Yao, T., Journel, A.G., 1998. Automatic modeling of (cross) covariance tables using fast fourier transform. Mathematical Geology 30, 589-615.

Yeh, H., Huang, Y., 2005. Parameter estimation for leaky aquifers using the extended kalman filter, and considering model and data measurement uncertainties. Journal of Hydrology 302, 28-45.

Zhang, T., Switzer, P., Journel, A., 2006. Filter-based classification of training image patterns for spatial simulation. Mathematical Geology 38, 6380 . 
Zhang, Y., Oliver, D., 2010. Improving the ensemble estimate of the kalman gain by bootstrap sampling. Mathematical Geosciences 42, 327-345.

Zhao, Y., Reynolds, A., Li, G., 2008. Generating facies maps by assimilating production data and seismic data with the ensemble kalman filter, in: SPE/DOE Symposium on Improved Oil Recovery.

Zheng, X., 2009. An adaptive estimation of forecast error covariance parameters for kalman filtering data assimilation. Advances in Atmospheric Sciences 26, 154-160.

Zhou, H., Gómez-Hernández, J., Hendricks Franssen, H., Li, L., 2011. An approach to handling non-gaussianity of parameters and state variables in ensemble kalman filtering. Advances in Water Resources 34, 844-864.

Zhou, H., Gómez-Hernández, J.J., Li, L., 2012. A pattern-search-based inverse method. Water Resources Research 48.

Zhou, H., Gómez-Hernández, J.J., Li, L., 2013. Inverse methods in hydrogeology: Evolution and recent trends. Advances in Water Resources

Zhou, H., Gómez-Hernández, J.J., Li, L., 2014. Inverse methods in hydrogeology: Evolution and recent trends. Advances in Water Resources 63, $22-37$. 


\section{A \\ Normal-score transform}

We have $N_{e}$ realizations, each one discretized into $N$ nodes. Let $K_{i, j}$ the conductivity for realization $i$ at node $j$. For each node, determine the experimental cumulative distribution function (cdf) from the set of values $\left\{K_{i, j}, i=1, \ldots, N_{e}\right\}$ :

$$
F_{j}(k)=\operatorname{Prob}(K \leq k), j=1, \ldots, N,
$$

these cdfs are generally non-parametric, and they are defined in tabular form by pairs of $\left(k, F_{j}(k)\right)$ values. Let $G(y)$ be the cumulative distribution function of a variable $y$ having a Gaussian distribution of zero mean and unit variance.

The normal-score transformation is given by:

$$
\widetilde{K_{i, j}}=G^{-1}\left(F_{j}\left(K_{i, j}\right)\right), \quad i=1, \ldots, N_{e} ; \quad j=1, \ldots, N .
$$

Similarly, the normal-score back transform is given by:

$$
K_{i, j}=F^{-1}\left(G\left(\widetilde{K_{i, j}}\right)\right), i=1, \ldots, N_{e} ; j=1, \ldots, N .
$$




